Network Working Group

Request for Comments: 4805

Obsoletes: 3895

Category: Standards Track
O. Nicklass, Ed. RAD Data Communications, Ltd.

\title{
Definitions of Managed Objects for the DS1, J1, E1, DS2, and E2 Interface Types
}

Status of This Memo

This document specifies an Internet standards track protocol for the Internet community, and requests discussion and suggestions for improvements. Please refer to the current edition of the "Internet Official Protocol standards" (STD 1) for the standardization state and status of this protocol. Distribution of this memo is unlimited.

Copyright Notice

Copyright (C) The IETF Trust (2007) .

Abstract

This memo defines a portion of the Management Information Base (MIB) for use with network management protocols in the Internet community. In particular, it describes objects used for managing DS1, J1, E1, DS2, and E2 interfaces. This document is a companion to the documents that define managed objects for the DS0, DS3/E3, and Synchronous Optical Network/Synchronous Digital Hierarchy (SONET/SDH) Interface Types.

This document obsoletes RFC 3895. 
Table of Contents

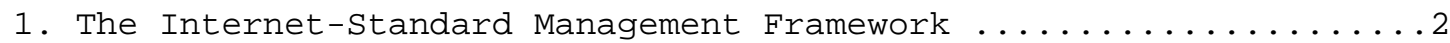

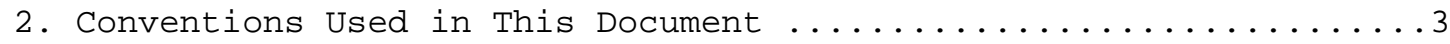

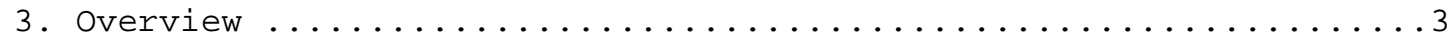

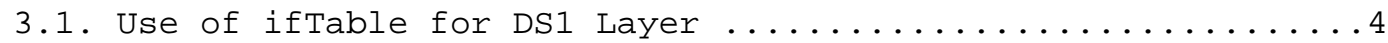

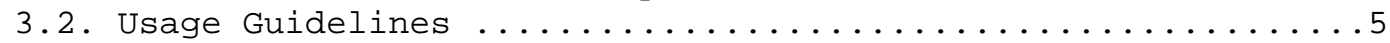

3.2.1. Usage of ifStackTable for Routers and DSUs .......5

3.2.2. Usage of ifStackTable for DS1/J1/E1 on DS2/E2 .....7

3.2.3. Usage of Channelization for DS3, DS1, DS0 .......8

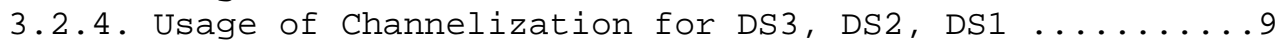

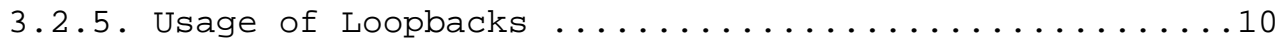

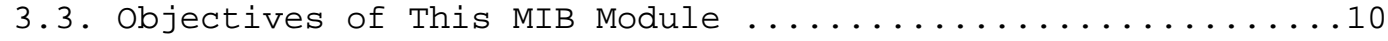

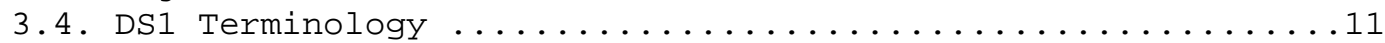

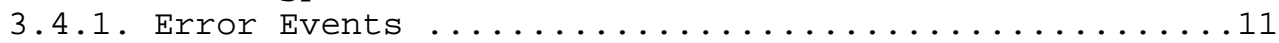

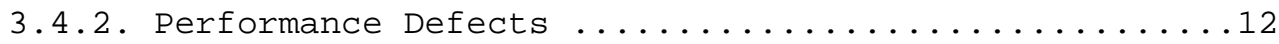

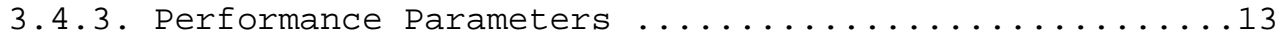

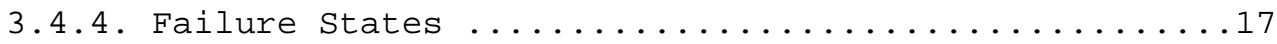

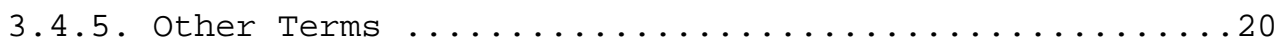

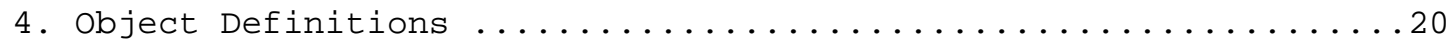

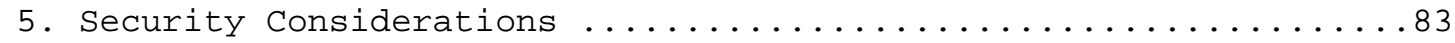

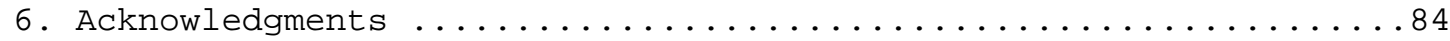

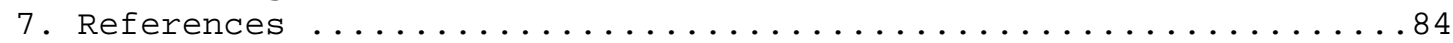

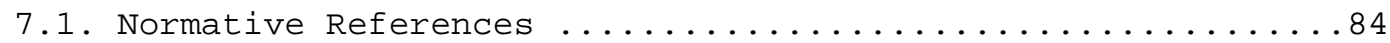

7.2. Informative References ...................... 86

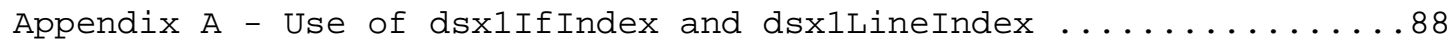

Appendix B - The Delay Approach to Unavailable Seconds .........90

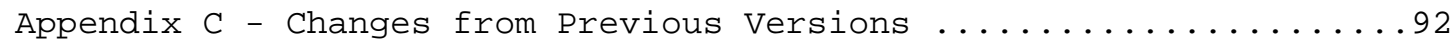

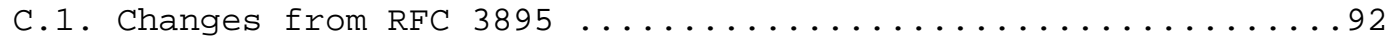

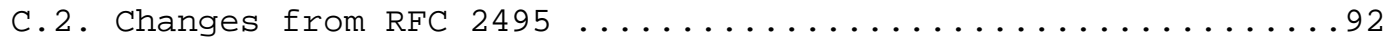

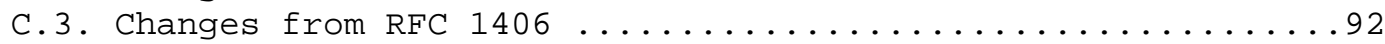

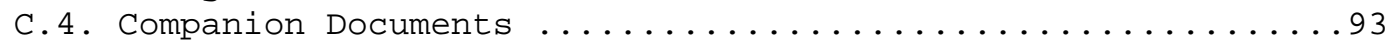

1. The Internet-Standard Management Framework

For a detailed overview of the documents that describe the current Internet-Standard Management Framework, please refer to section 7 of RFC 3410 [RFC3410].

Managed objects are accessed via a virtual information store, termed the Management Information Base or MIB. MIB objects are generally accessed through the Simple Network Management Protocol (SNMP). Objects in the MIB are defined using the mechanisms defined in the Structure of Management Information (SMI). This memo specifies a MIB module that is compliant to the SMIV2, which is described in STD 58, RFC 2578 [RFC2578], STD 58, RFC 2579 [RFC2579] and STD 58, RFC 2580 [RFC2580]. 
2. Conventions Used in This Document

The key words "MUST", "MUST NOT", "REQUIRED", "SHALL", "SHALL NOT", "SHOULD", "SHOULD NOT", "RECOMMENDED", "MAY", and "OPTIONAL" in this document are to be interpreted as described in RFC 2119 [RFC2119].

\section{Overview}

These objects are used when the particular media being used to realize an interface is a DS1/J1/E1/DS2/E2 interface. At present, this applies to the following value of the iftype variable in the Internet-standard MIB:

ds 1 (18)

The definitions contained herein are based on the AT\&T T-1 Superframe (a.k.a. D4) [ANSI-T1.107] and Extended Superframe (ESF) formats [AT\&T-UM-305], [AT\&T-TR-54016], the latter of which conforms to ANSI specifications [ANSI-T1.403], and the CCITT Recommendations [CCITT-G.703], [ITU-T-G.704], referred to as E1 for the rest of this memo. J1 refers to the definition presented in [JT-G704], [JT-G706], and $[\mathrm{JT}-\mathrm{I} 431]$.

The various DS1, J1, and E1 line disciplines are similar enough that separate MIBs are unwarranted, although there are some differences. For example, Loss of Frame is defined more rigorously in the ESF specification than in the D4 specification, or Yellow Alarm generation and detection are a bit different between $\mathrm{T} 1$ and $\mathrm{J} 1$ but in both examples, there is definition in both related lines. Therefore, interface types el(19) and g703at2mb(67) have been obsoleted and there is also no need for special type for J1.

Where it is necessary to distinguish between the flavors of E1 with and without Cyclic Redundancy Check (CRC), E1-CRC denotes the "with CRC" form (G.704 Table 5B) and E1-noCRC denotes the "without CRC" form (G.704 Table 5A). 
3.1. Use of ifTable for DS1 Layer

Only the ifGeneralInformationGroup needs to be supported.

\begin{tabular}{|c|c|}
\hline $\begin{array}{c}\text { iftable Object } \\
====================\text {. }\end{array}$ & $\begin{array}{l}\text { Use for DS1 Layer } \\
\text { = }\end{array}$ \\
\hline ifIndex & Interface index. \\
\hline ifDescr & See interfaces MIB [RFC2863]. \\
\hline iftype & ds $1(18)$ \\
\hline ifspeed & $\begin{array}{l}\text { Speed of line rate } \\
\text { DS1 - } 1544000 \\
\text { J1 - } 1544000 \\
\text { E1 - } 2048000 \\
\text { DS2 - } 6312000 \\
\text { E2 - } 8448000\end{array}$ \\
\hline ifPhysAddress & $\begin{array}{l}\text { The value of the Circuit Identifier. } \\
\text { If no Circuit Identifier has been assigned, } \\
\text { this object should have an octet string } \\
\text { with zero length. }\end{array}$ \\
\hline ifAdminstatus & See interfaces MIB [RFC2863]. \\
\hline ifoperstatus & See interfaces MIB [RFC2863]. \\
\hline ifLastChange & See interfaces MIB [RFC2863]. \\
\hline ifName & See interfaces MIB [RFC2863]. \\
\hline ifLinkUpDownTrapEn & lable set to enabled(1). \\
\hline ifHighSpeed & $\begin{array}{l}\text { Speed of line in mega-bits per second } \\
(2,6 \text {, or } 8) \text {. }\end{array}$ \\
\hline ifConnectorPresent & $\begin{array}{l}\text { Set to true(1) normally, except for } \\
\text { cases such as DS1/E1 over AAL1/ATM where } \\
\text { false(2) is appropriate. }\end{array}$ \\
\hline
\end{tabular}




\subsection{Usage Guidelines}

\subsubsection{Usage of ifStackTable for Routers and DSUs}

The object dsxlIfIndex has been deprecated. This object previously allowed a very special proxy situation to exist for routers and Channel Service Units (CSUs). This section now describes how to use the ifstackTable to represent this relationship.

The paragraphs discussing dsxlIfIndex and dsxlLineIndex have been preserved in Appendix A for informational purposes.

The ifStackTable is used in the proxy case to represent the association between pairs of interfaces, i.e., this T1 is attached to that T1. This use is consistent with the use of the ifstackTable to show the association between various sub-layers of an interface. In both cases, entire PDUs are exchanged between the interface pairs -in the case of a T1, entire T1 frames are exchanged; in the case of PPP and High-Level Data Link Control (HDLC), entire HDLC frames are exchanged. This usage is not meant to suggest the use of the ifstackTable to represent Time Division Multiplexing (TDM) connections in general.

External and Internal interface scenario: the SNMP agent resides on a host external from the device supporting DS1 interfaces (e.g., a router). The agent represents both the host and the DS1 device. 
Example:

A shelf full of CSUs connected to a router. An SNMP agent residing on the router proxies for itself and the CSU. The router has also an Ethernet interface:

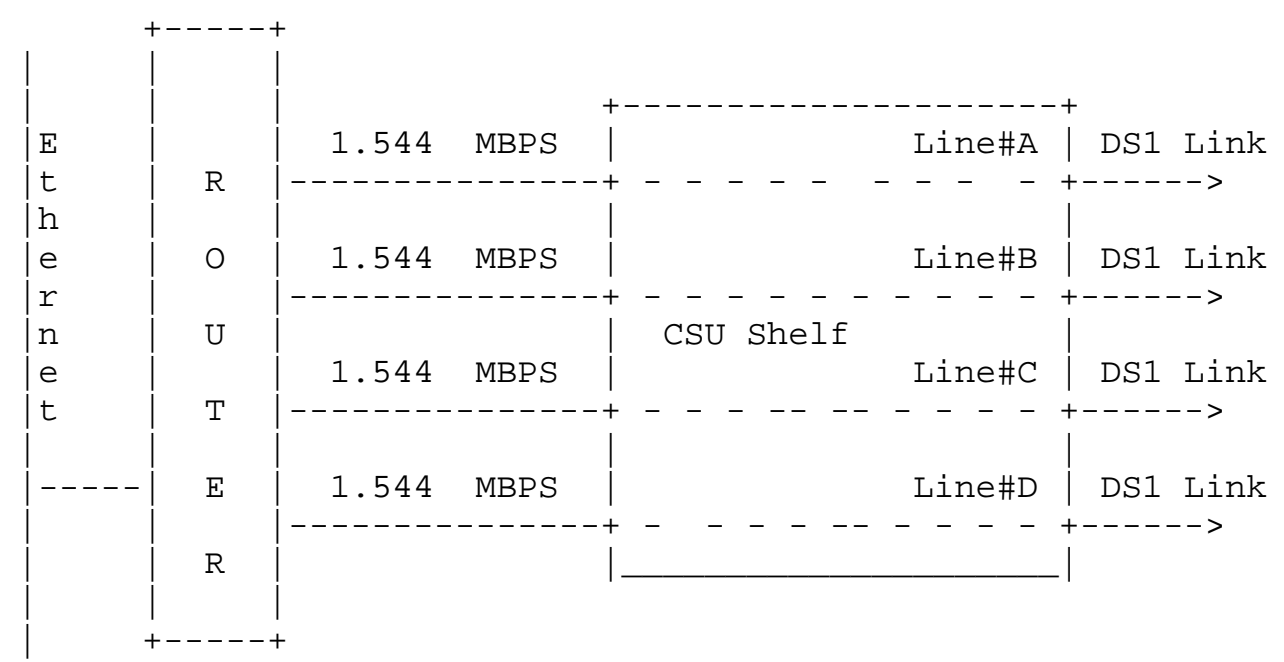

The assignment of the index values could, for example, be as follows:

$\begin{array}{ll}\text { ifIndex } & \text { Description } \\ 1 & \text { Ethernet } \\ 2 & \text { Line\#A Router } \\ 3 & \text { Line\#B Router } \\ 4 & \text { Line\#C Router } \\ 5 & \text { Line\#D Router } \\ 6 & \text { Line\#A CSU Router } \\ 7 & \text { Line\#B CSU Router } \\ 8 & \text { Line\#C CSU Router } \\ 9 & \text { Line\#D CSU Router } \\ 10 & \text { Line\#A CSU Network } \\ 11 & \text { Line\#B CSU Network } \\ 12 & \text { Line\#C CSU Network } \\ 13 & \text { Line\#D CSU Network }\end{array}$

The ifstackTable is then used to show the relationships between the various DS1 interfaces. 


$\begin{array}{lc}\text { ifStackTable } & \text { Entries } \\ \text { HigherLayer } & \text { LowerLayer } \\ 2 & 6 \\ 3 & 7 \\ 4 & 8 \\ 5 & 9 \\ 6 & 10 \\ 7 & 11 \\ 8 & 12 \\ 9 & 13\end{array}$

If the CSU shelf is managed by itself by a local SNMP agent, the situation would be identical, except the Ethernet and the four router interfaces are deleted. Interfaces would also be numbered from 1 to 8.

$\begin{array}{ll}\text { ifIndex } & \text { Description } \\ 1 & \text { Line\#A CSU Router } \\ 2 & \text { Line\#B CSU Router } \\ 3 & \text { Line\#C CSU Router } \\ 4 & \text { Line\#D CSU Router } \\ 5 & \text { Line\#A CSU Network } \\ 6 & \text { Line\#B CSU Network } \\ 7 & \text { Line\#C CSU Network } \\ 8 & \text { Line\#D CSU Network } \\ \text { ifStackTable Entries } \\ \text { HigherLayer } \\ 1 & \text { LowerLayer } \\ 2 & 5 \\ 3 & 6\end{array}$

3.2.2. Usage of ifstackTable for DS1/J1/E1 on DS2/E2

An example is given of how DS1/J1/E1 interfaces are stacked on DS2/E2 interfaces. It is not necessary nor is it always desirable to represent DS2 interfaces. If this is required, the following stacking should be used. All iftypes are ds1. The DS2 is determined by examining ifspeed or dsxllineType. 


\begin{tabular}{|c|c|c|}
\hline ifIndex & \multicolumn{2}{|c|}{ Description } \\
\hline 1 & DS1 & $\# 1$ \\
\hline 2 & DS1 & $\# 2$ \\
\hline 3 & DS1 & \#3 \\
\hline 4 & DS1 & $\# 4$ \\
\hline 5 & DS2 & \\
\hline \multicolumn{2}{|c|}{ ifStackTable } & Entries \\
\hline \multicolumn{2}{|c|}{ HigherLayer } & LowerLayer \\
\hline \multicolumn{2}{|c|}{1} & 5 \\
\hline \multicolumn{2}{|l|}{2} & 5 \\
\hline \multicolumn{2}{|l|}{3} & 5 \\
\hline \multicolumn{2}{|l|}{4} & 5 \\
\hline
\end{tabular}

\subsubsection{Usage of Channelization for DS3, DS1, DSO}

An example is given here to explain the channelization objects in the DS3, DS1, and DSO MIBs to help the implementer use the objects correctly. Treatment of E3 and E1 would be similar, with the number of DSOs being different depending on the framing of the E1.

Assume that a DS3 (with ifIndex 1) is channelized into DS1s (without DS2s). The object dsx3Channelization is set to enabledDs1. There will be 28 DSIs in the ifTable. Assume the entries in the iftable for the DSIs are created in channel order and the ifIndex values are 2 through 29. In the DS1 MIB, there will be an entry in the dsx1ChanMappingTable for each DS1. The entries will be as follows:

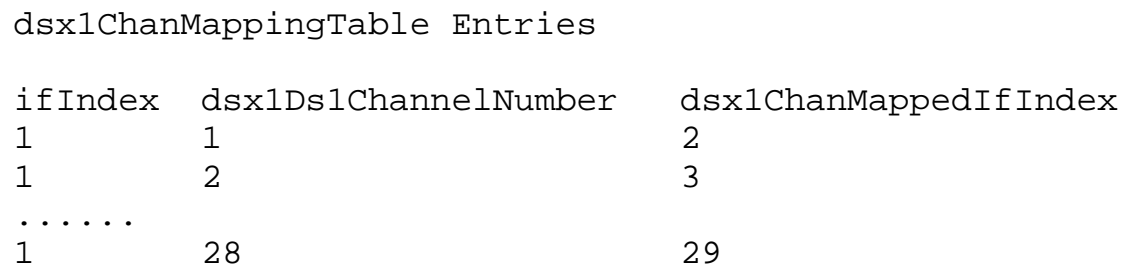

In addition, the DS1s are channelized into DSOs. The object dsx1Channelization is set to enabledDSO for each DS1. When this object is set to this value, 24 DSOs are created by the agent. There will be 24 DSOs in the ifTable for each DS1. If the dsxichannelization is set to disabled, the 24 DSOs are destroyed.

Assume the entries in the iftable are created in channel order and the ifIndex values for the DSOs in the first DS1 are 30 through 53. In the DSO MIB, there will be an entry in the dsx0ChanMappingTable for each DSO. The entries will be as follows: 


\begin{tabular}{|c|c|c|}
\hline ifIndex & dsx0Ds0ChannelNumber & dsx0ChanMappedIfIndex \\
\hline 2 & 1 & 30 \\
\hline 2 & 2 & 31 \\
\hline$\dot{2}^{\cdots \cdots}$ & 24 & 53 \\
\hline
\end{tabular}

\subsubsection{Usage of Channelization for DS3, DS2, DS1}

An example is given here to explain the channelization objects in the DS3 and DS1 MIBs to help the implementer use the objects correctly.

Assume that a DS3 (with ifIndex 1) is channelized into DS2s. The object dsx3Channelization [RFC3896] is set to enabledDs2. There will be 7 DS2s (ifType of DS1) in the ifTable. Assume the entries in the ifTable for the DS2s are created in channel order and the ifIndex values are 2 through 8. In the DS1 MIB, there will be an entry in the dsx1ChanMappingTable for each DS2. The entries will be as follows:

\begin{tabular}{|c|c|c|}
\hline ifIndex & dsx1Ds1ChannelNumber & dsx1ChanMappedIfIndex \\
\hline 1 & 1 & 2 \\
\hline 1 & 2 & 3 \\
\hline$\ddot{1} \cdots \cdots$ & 7 & 8 \\
\hline
\end{tabular}

In addition, the DS2s are channelized into DS1s. The object dsx1Channelization is set to enabledDS1 for each DS2. There will be 4 DS1s in the ifTable for each DS2. Assume the entries in the iftable are created in channel order and the ifIndex values for the DS1s in the first DS2 are 9 through 12, then 13 through 16 for the second DS2, and so on. In the DS1 MIB, there will be an entry in the dsxlChanMappingTable for each DS1. The entries will be as follows:

$\begin{array}{lll}\text { dsxiChanMappingTable Entries } \\ \text { ifIndex } & \text { dsxids1ChannelNumber } & \text { dsxiChanMappedIfIndex } \\ 2 & 1 & 9 \\ 2 & 2 & 10 \\ 2 & 3 & 11 \\ 2 & 4 & 12 \\ 3 & 1 & 13 \\ 3 & 2 & 14 \\ 8 & & 36\end{array}$




\subsubsection{Usage of Loopbacks}

This section discusses the behavior of objects related to loopbacks.

The object dsxlloopbackConfig represents the desired state of loopbacks on this interface. Using this object, a manager can request

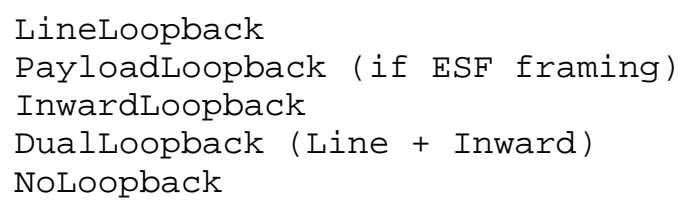

The remote end can also request loopbacks either through the Facility Data Link (FDL) channel if ESF or inband if D4. The loopbacks that can be requested this way are

LineLoopback

PayloadLoopback (if ESF framing) NoLoopback

To model the current state of loopbacks on a DS1 interface, the object dsxlLoopbackstatus defines which loopback is currently applied to an interface. This object, which is a bitmap, will have bits turned on that reflect the currently active loopbacks on the interface as well as the source of those loopbacks.

The following restrictions/rules apply to loopbacks:

The far end cannot undo loopbacks set by a manager.

A manager can undo loopbacks set by the far end.

Both a line loopback and an inward loopback can be set at the same time. Only these two loopbacks can co-exist and either one may be set by the manager or the far end. A LineLoopback request from the far end is incremental to an existing Inward loopback established by a manager. When a NoLoopback is received from the far end in this case, the InwardLoopback remains in place.

\subsection{Objectives of This MIB Module}

There are numerous things that could be included in a MIB for DS1 signals: the management of multiplexers, CSUs, Data Service Units (DSUs), and the like. The intent of this document is to facilitate the common management of all devices with DS1, J1, E1, DS2, or E2 interfaces. As such, a design decision was made up front to very 
closely align the MIB with the set of objects that can generally be read from these types of devices that are currently deployed.

J2 interfaces are not supported by this MIB.

3.4. DS1 Terminology

The terminology used in this document to describe error conditions on a DS1 interface as monitored by a DS1 device are based on the latest ANSI T1.231 standard [ANSI-T1.231]. If the definition in this document does not match the definition in the ANSI T1.231 document, the implementer should follow the definition described in this document.

\subsubsection{Error Events}

Bipolar Violation (BPV) Error Event

A BPV error event for an AMI-coded (AMI stands for Alternate Mark Inversion) signal is the occurrence of a pulse of the same polarity as the previous pulse (see T1.231, Section 4.2.1.1.1). A BPV error event for a B8ZS- or HDB3-coded signal is the occurrence of a pulse of the same polarity as the previous pulse without being a part of the zero substitution code.

Excessive Zeroes (EXZ) Error Event

An Excessive Zeroes error event for an AMI-coded signal is the occurrence of more than fifteen contiguous zeroes (see T1.231 Section 4.2.1.1.2). For a B8ZS-coded signal, the defect occurs when more than seven contiguous zeroes are detected.

Line Coding Violation (LCV) Error Event

A Line Coding Violation (LCV) is the occurrence of either a Bipolar Violation (BPV) or Excessive Zeroes (EXZ) error event. (Also known as CV-L; see T1.231, Section 4.6.1.1.)

Path Coding Violation (PCV) Error Event

A Path Coding Violation error event is a frame synchronization bit error in the D4 and E1-noCRC formats, or a CRC or frame synch. bit error in the ESF and E1-CRC formats. (Also known as CV-P; see T1.231, Section 4.6.2.1.)

Controlled Slip (CS) Error Event

A Controlled Slip is the replication or deletion of the payload bits of a DS1 frame (see T1.231, Section 4.2.1.2.3). A Controlled Slip may be performed when there is a difference between the timing of a synchronous receiving terminal and the received signal. A Controlled Slip does not cause an out of Frame defect. 


\subsubsection{Performance Defects}

Out of Frame (OOF) Defect

An OOF defect is the occurrence of a particular density of Framing Error events (see T1.231, Section 4.2.2.2.1).

For DS1 links, an Out of Frame defect is declared when the receiver detects two or more framing errors within a 3-msec period for ESF signals and $0.75 \mathrm{msec}$ for D4 signals, or two or more errors out of five or fewer consecutive framing bits.

For El links, an Out of Frame defect is declared when three consecutive frame alignment signals have been received with an error (see G.706, Section 4.1 [CCITT-G.706]).

For DS2 links, an Out of Frame defect is declared when seven or more consecutive errored framing patterns (four multiframe) are received. The OOF is cleared when three or more consecutive correct framing patterns are received.

Once an Out of Frame Defect is declared, the framer starts searching for a correct framing pattern. The Out of Frame defect ends when the signal is in-frame.

In-frame occurs when there are fewer than two frame bit errors within a 3-msec period for ESF signals and 0.75 msec for D 4 signals.

For E1 links, in-frame occurs when a) in frame $\mathrm{N}$ the frame alignment signal is correct and b) in frame $\mathrm{N}+1$ the frame alignment signal is absent (i.e., bit 2 in TSO is a one) and c) in frame $N+2$ the frame alignment signal is present and correct (see G.704, Section 4.1).

Alarm Indication Signal (AIS) Defect

For D4 and ESF links, the 'all ones' condition is detected at a DS1 line interface upon observing an unframed signal with a one's density of at least $99.9 \%$ present for a time equal to or greater than $\mathrm{T}$, where $3 \mathrm{~ms}<=\mathrm{T}<=75 \mathrm{~ms}$. The AIS is terminated upon observing a signal not meeting the one's density or the unframed signal criteria for a period equal to or greater than $\mathrm{T}$ (see G.775, Section 5.4).

For El links, the 'all-ones' condition is detected at the line interface as a string of 512 bits containing fewer than three zero bits (see 0.162 [ITU-T-0.162], Section 3.3.2). 
For DS2 links, the DS2 AIS shall be sent from the NT1 to the user to indicate a loss of the 6,312-kbps frame capability on the network side. The DS2 AIS is defined as a bit array of $6,312 \mathrm{kbps}$ in which all binary bits are set to ' ${ }^{\prime}$ '.

The DS2 AIS detection and removal shall be implemented according to ITU-T Draft Recommendation G.775 [ITU-T-G.775] Section 5.5:

- a DS2 AIS defect is detected when the incoming signal has two or less zeroes in a sequence of 3156 bits $(0.5 \mathrm{~ms})$.

- a DS2 AIS defect is cleared when the incoming signal has three or more zeroes in a sequence of 3156 bits $(0.5 \mathrm{~ms})$.

\subsubsection{Performance Parameters}

All performance parameters are accumulated in 15-minute intervals, and up to 96 intervals (24 hours' worth) are kept by an agent. Fewer than 96 intervals of data will be available if the agent has been restarted within the last 24 hours. In addition, there is a rolling 24-hour total of each performance parameter. Performance parameters continue to be collected when the interface is down.

There is no requirement for an agent to ensure a fixed relationship between the start of a 15-minute interval and any wall clock; however, some agents may align the 15-minute intervals with quarter hours.

Performance parameters are of types PerfCurrentCount, PerfintervalCount, and PerfTotalcount. These textual conventions are all Gauge32, and they are used because it is possible for these objects to decrease. Objects may decrease when Unavailable Seconds occur across a 15-minute interval boundary. See Unavailable Second discussion later in this section.

Line Errored Second (LES)

A Line Errored Second is a second in which one or more Line Coding Violation error events were detected. (Also known as ES-L; see T1.231, section 4.6.1.2.)

Controlled Slip second (CSS)

A Controlled Slip second is a one-second interval containing one or more controlled slips (see T1.231, Section 4.6.2.9). This is not incremented during an Unavailable Second. 
Errored Second (ES)

For ESF and E1-CRC links, an Errored Second is a second with one or more Path Coding Violations OR one or more Out of Frame defects OR one or more Controlled Slip events OR a detected AIS defect. (See T1.231, Section 4.6.2.2 and G.826 [ITU-T-G.826], Section B. 1).

For D4 and El-noCRC links, the presence of Bipolar Violations also triggers an Errored Second.

This is not incremented during an Unavailable Second.

Bursty Errored Second (BES)

A Bursty Errored Second (also known as Errored Second type B in T1.231, Section 4.6.2.4) is a second with fewer than 320 and more than 1 Path Coding Violation error events, no Severely Errored Frame defects, and no detected incoming AIS defects. Controlled Slips are not included in this parameter.

This is not incremented during an Unavailable Second. It applies to ESF signals only.

Severely Errored Second (SES)

A Severely Errored Second for ESF signals is a second with 320 or more Path Coding Violation error events OR one or more out of Frame defects OR a detected AIS defect (see T1.231, section $4.6 .2 .5)$.

For El-CRC signals, a Severely Errored Second is a second with 832 or more Path Coding Violation error events OR one or more out of Frame defects.

For E1-noCRC signals, a Severely Errored Second is 2048 LCVs or more.

For D4 signals, a Severely Errored Second is a count of one-second intervals with Framing Error events, or an OOF defect, or 1544 LCVs or more.

Controlled Slips are not included in this parameter.

This is not incremented during an Unavailable Second.

Severely Errored Framing Second (SEFS)

An Severely Errored Framing Second is a second with one or more Out of Frame defects OR a detected AIS defect. (Also known as SAS-P (SEF/AIS second); see T1.231, Section 4.6.2.6.) 
Degraded Minutes

A Degraded Minute is one in which the estimated error rate exceeds 1E-6 but does not exceed 1E-3 (see G.821 [CCITT-G.821]).

Degraded Minutes are determined by collecting all of the Available Seconds, removing any Severely Errored Seconds, grouping the result in 60-second long groups, and counting a 60-second long group (a.k.a. minute) as degraded if the cumulative errors during the seconds present in the group exceed 1E-6. Available seconds are merely those seconds that are not Unavailable as described below.

Unavailable Second (UAS) Unavailable Seconds (UASS) are calculated by counting the number of seconds that the interface is unavailable. The DS1 interface is said to be unavailable from the onset of 10 contiguous SESs, or the onset of the condition leading to a failure (see Failure States). If the condition leading to the failure was immediately preceded by one or more contiguous SESs, then the DS1 interface unavailability starts from the onset of these SESs. Once unavailable, and if no failure is present, the DS1 interface becomes available at the onset of 10 contiguous seconds with no SESs. Once unavailable, and if a failure is present, the DS1 interface becomes available at the onset of 10 contiguous seconds with no SESs, if the failure clearing time is less than or equal to 10 seconds. If the failure clearing time is more than 10 seconds, the DS1 interface becomes available at the onset of 10 contiguous seconds with no SESs, or the onset period leading to the successful clearing condition, whichever occurs later. With respect to the DS1 error counts, all counters are incremented while the DSI interface is deemed available. While the interface is deemed unavailable, the only count that is incremented is UASs.

Note that this definition implies that the agent cannot determine until after a 10-second interval has passed whether a given onesecond interval belongs to available or unavailable time. If the agent chooses to update the various performance statistics in real time, then it must be prepared to retroactively reduce the ES, BES, SES, and SEFS counts by 10 and increase the UAS count by 10 when it determines that available time has been entered. It must also be prepared to adjust the PCV count and the DM count as necessary since these parameters are not accumulated during unavailable time. It must be similarly prepared to retroactively decrease the UAS count by 10 and increase the ES, BES, and DM counts as necessary upon entering available time. A special case exists when the 10-second period leading to available or unavailable time crosses a 900-second statistics window boundary, as the foregoing description implies that the ES, BES, SES, SEFS, 
DM, and UAS counts the PREVIOUS interval must be adjusted. In this case, successive GETs of the affected dsxilntervalSESs and dsxlintervalUASs objects will return differing values if the first GET occurs during the first few seconds of the window.

The agent may instead choose to delay updates to the various statistics by 10 seconds in order to avoid retroactive adjustments to the counters. A way to do this is sketched in Appendix B.

In any case, a linkDown trap shall be sent only after the agent has determined for certain that the unavailable state has been entered, but the time on the trap will be that of the first UAS (i.e., 10 seconds earlier). A linkUp trap shall be handled similarly.

According to ANSI T1.231, unavailable time begins at the onset of 10 contiguous severely errored seconds -- that is, unavailable time starts with the first of the 10 contiguous SESs. Also, while an interface is deemed unavailable all counters for that interface are frozen except for the UAS count. It follows that an implementation that strictly complies with this standard must not increment any counters other than the UAS count -- even temporarily -- as a result of anything that happens during those 10 seconds. Since changes in the signal state lag the data to which they apply by 10 seconds, an ANSI-compliant implementation must pass the one-second statistics through a 10-second delay line prior to updating any counters. That can be done by performing the following steps at the end of each one-second interval.

i) Read near/far end CV counter and alarm status flags from the hardware.

ii) Accumulate the CV counts for the preceding second and compare them to the ES and SES threshold for the layer in question. Update the signal state and shift the one-second CV counts and ES/SES flags into the 10-element delay line. Note that far-end one-second statistics are to be flagged as "absent" during any second in which there is an incoming defect at the layer in question or at any lower layer.

iii) Update the current interval statistics using the signal state from the previous update cycle and the one-second CV counts and ES/SES flags shifted out of the 10-element delay line.

This approach is further described in Appendix B. 


\subsubsection{Failure states}

The following failure states are received, or detected failures, that are reported in the dsxlLinestatus object. When a DS1 interface would, if ever, produce the conditions leading to the failure state is described in the appropriate specification.

Far End Alarm Failure

The Far End Alarm failure is also known as "Yellow Alarm" in the DS1 and J1 cases, "Distant Alarm" in the E1 case, and "Remote Alarm" in the DS2 case.

For D4 links, the Far End Alarm failure is declared when bit 6 of all channels has been zero for at least $335 \mathrm{~ms}$ and is cleared when bit 6 of at least one channel is non-zero for a period $T$, where $T$ is usually less than one second and always less than five seconds. The Far End Alarm failure is not declared for D4 links when a Loss of Signal is detected. In $\mathrm{J} 1$ the 12 th $\mathrm{F}$-bit is set to 1 .

For ESF links, the Far End Alarm failure is declared if the Yellow Alarm signal pattern occurs in at least seven out of ten contiguous 16-bit pattern intervals and is cleared if the Yellow Alarm signal pattern does not occur in ten contiguous 16-bit signal pattern intervals. For DS1 the patterns is FFOO and for J1 the pattern is FFFF.

For El links, the Far End Alarm failure is declared when bit 3 of time-slot zero is received set to one on two consecutive occasions. The Far End Alarm failure is cleared when bit 3 of time-slot zero is received set to zero.

For DS2 links, if a loss of frame alignment (LOF or LOS) and/or DS2 AIS condition is detected, the RAI signal shall be generated and transmitted to the remote side.

The Remote Alarm Indication (RAI) signal is defined on m-bits as a repetition of the 16-bit sequence consisting of eight binary ' $1 \mathrm{~s}^{\prime}$ and eight binary ' $0 s^{\prime}$ in m-bits(1111111100000000). When the RAI signal is not sent (in normal operation), the HDLC flag pattern (01111110) in the m-bit is sent.

The RAI failure is detected when 16 or more consecutive RAIpatterns (1111111100000000) are received. The RAI failure is cleared when 4 or more consecutive incorrect-RAI-patterns are received. 
Alarm Indication Signal (AIS) Failure

The Alarm Indication Signal failure is declared when an AIS defect is detected at the input and the AIS defect still exists after the Loss of Frame failure (which is caused by the unframed nature of the 'all-ones' signal) is declared. The AIS failure is cleared when the Loss of Frame failure is cleared. (See T1.231, Section $4.3 .1 .2 .2)$.

An AIS defect at a 6312-kbit/s (G.704) interface is detected when the incoming signal has two or less zeroes in a sequence of 3156 bits $(0.5 \mathrm{~ms})$.

The AIS signal defect is cleared when the incoming signal has three $\{3\}$ or more zeroes in a sequence of 3156 bits $(0.5 \mathrm{~ms})$.

Loss of Frame (LOF) Failure For DS1 links, the Loss of Frame failure is declared when an OOF or LOS defect has persisted for $\mathrm{T}$ seconds, where $2<=\mathrm{T}<=10$. The Loss of Frame failure is cleared when there have been no OOF or LOS defects during a period $\mathrm{T}$ where $0<=\mathrm{T}<=20$. Many systems will perform "hit integration" within the period $T$ before declaring or clearing the failure; e.g., see TR 62411 [AT\& T-TR-62411].

For E1 links, the Loss of Frame failure is declared when an OOF defect is detected.

Loss Of Signal (LOS) Failure For DS1, the Loss of Signal failure is declared upon observing 175 +/- 75 contiguous pulse positions with no pulses of either positive or negative polarity. The LOS failure is cleared upon observing an average pulse density of at least $12.5 \%$ over a period of 175 +/- 75 contiguous pulse positions starting with the receipt of a pulse.

For El links, the Loss of Signal failure is declared when greater than 10 consecutive zeroes are detected (see 0.162, Section $3.4 .4)$.

A LOS defect at $6312 \mathrm{kbit} / \mathrm{s}$ interfaces is detected when the incoming signal has "no transitions", i.e., when the signal level is less than or equal to a signal level of $35 \mathrm{~dB}$ below nominal, for $\mathrm{N}$ consecutive pulse intervals, where $10<=\mathrm{N}<=255$.

The LOS defect is cleared when the incoming signal has "transitions", i.e., when the signal level is greater than or equal to a signal level of $9 \mathrm{~dB}$ below nominal, for $\mathrm{N}$ consecutive pulse intervals, where $10<=N<=255$. 
A signal with "transitions" corresponds to a G.703-compliant signal.

Loopback Pseudo-Failure

The Loopback Pseudo-Failure is declared when the near-end

equipment has placed a loopback (of any kind) on the DS1. This allows a management entity to determine from one object whether the DS1 can be considered to be in service or not (from the point of view of the near-end equipment).

TS16 Alarm Indication Signal Failure For E1 links, the TS16 Alarm Indication Signal failure is declared when time-slot 16 is received as all ones for all frames of two consecutive multiframes (see G.732, Section 4.2.6). This condition is never declared for DS1.

Loss of Multiframe Failure

The Loss of Multiframe failure is declared when two consecutive multiframe alignment signals (bits 4 through 7 of TS16 of frame 0) have been received with an error. The Loss of Multiframe failure is cleared when the first correct multiframe alignment signal is received. The Loss of Multiframe failure can only be declared for E1 links operating with G.732 [CCITT-G.732] framing (sometimes called "Channel Associated Signalling" mode).

Far End Loss of Multiframe Failure The Far End Loss of Multiframe failure is declared when bit 2 of TS16 of frame 0 is received set to one on two consecutive occasions. The Far End Loss of Multiframe failure is cleared when bit 2 of TS16 of frame 0 is received set to zero. The Far End Loss of Multiframe failure can only be declared for El links operating in "Channel Associated Signalling" mode (see G.732).

DS2 Payload AIS Failure

The DS2 Payload AIS failure is declared when the incoming signal of the 6,312-kbps frame payload (time-slots 1 through 96) has two or less zeroes in a sequence of 3072 bits $(0.5 \mathrm{~ms})$. The DS2 Payload AIS is cleared when the incoming signal of the 6,312-kbps frame payload has three or more zeroes in a sequence of 3072 bits $(0.5 \mathrm{~ms})$.

DS2 Performance Threshold Failure DS2 Performance Threshold failure monitors equipment performance and is based on the CRC (Cyclic Redundancy Check) procedure defined in G.704.

The DS2 Performance Threshold failure is declared when the bit error ratio exceeds $10^{\wedge}-4$ (Performance Threshold), and the DS2 
Performance Threshold failure is cleared when the bit error ratio decreases to less than $10^{\wedge}-6 . "$

\subsubsection{Other Terms}

Circuit Identifier

This is a character string specified by the circuit vendor and is useful when communicating with the vendor during the troubleshooting process (see M.1400 [ITU-T-M.1400] for additional information).

\section{Proxy}

In this document, the word proxy is meant to indicate an application that receives SNMP messages and replies to them on behalf of the devices that implement the actual DS1/E1 interfaces. The proxy may have already collected the information about the DS1/J1/E1 interfaces into its local database and may not necessarily forward the requests to the actual DS1/J1/E1 interface. It is expected in such an application that there are periods of time where the proxy is not communicating with the DS1/J1/E1 interfaces. In these instances, the proxy will not necessarily have up-to-date configuration information and will most likely have missed the collection of some statistics data. Missed statistics data collection will result in invalid data in the interval table.

4. Object Definitions

DS1-MIB DEFINITIONS : := BEGIN

IMPORTS

MODULE-IDENTITY, OBJECT-TYPE, NOTIFICATION-TYPE, transmission

FROM SNMPV2-SMI -- [RFC2578]

Displaystring, Timestamp, Truthvalue

FROM SNMPV2-TC -- [RFC2579]

MODULE-COMPLIANCE, OBJECT-GROUP, NOTIFICATION-GROUP

FROM SNMPV2-CONF -- [RFC2580]

InterfaceIndex, ifIndex

FROM IF-MIB -- [RFC2863]

PerfCurrentCount, PerfIntervalCount, PerfTotalcount

FROM PerfHist-TC-MIB; -- [RFC3593]

dS1 MODULE-IDENTITY

LAST-UPDATED "200703050000Z"

ORGANIZATION "IETF ATOM MIB Working Group" 


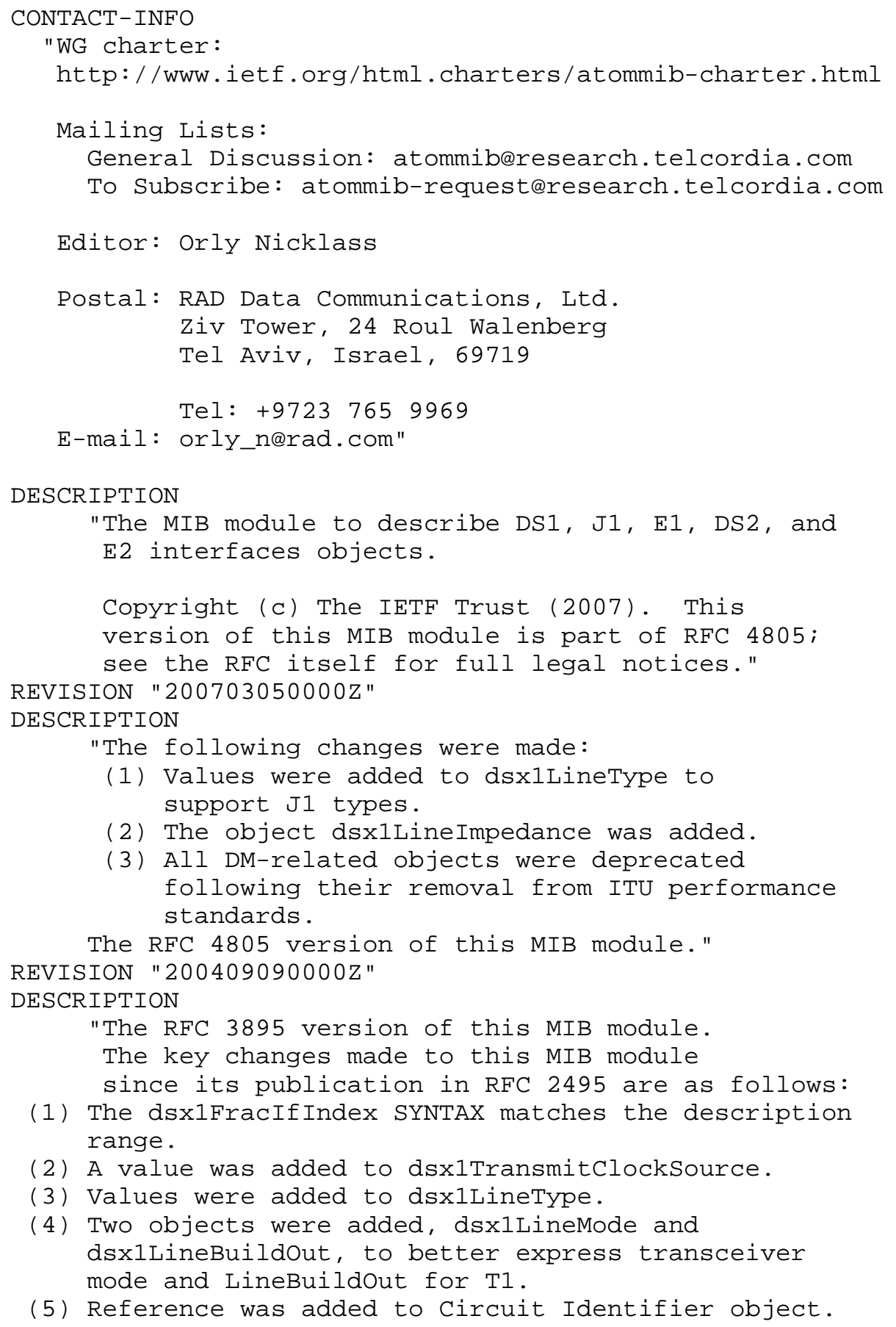

(2) A value was added to dsxitransmitclockSource.

(3) Values were added to dsxlLineType.

(4) Two objects were added, dsxllineMode and dsxlLineBuildout, to better express transceiver mode and LineBuildout for $\mathrm{T} 1$.

(5) Reference was added to Circuit Identifier object. 
(6) Align the DESCRIPTION clauses of few statistic objects with the near-end definition, with the far-end definition, and with RFC 3593.

(7) Changes in Compliance Statements to include new objects.

(8) A typographical error in dsx2E2 was fixed; the new name is dsx1E2."

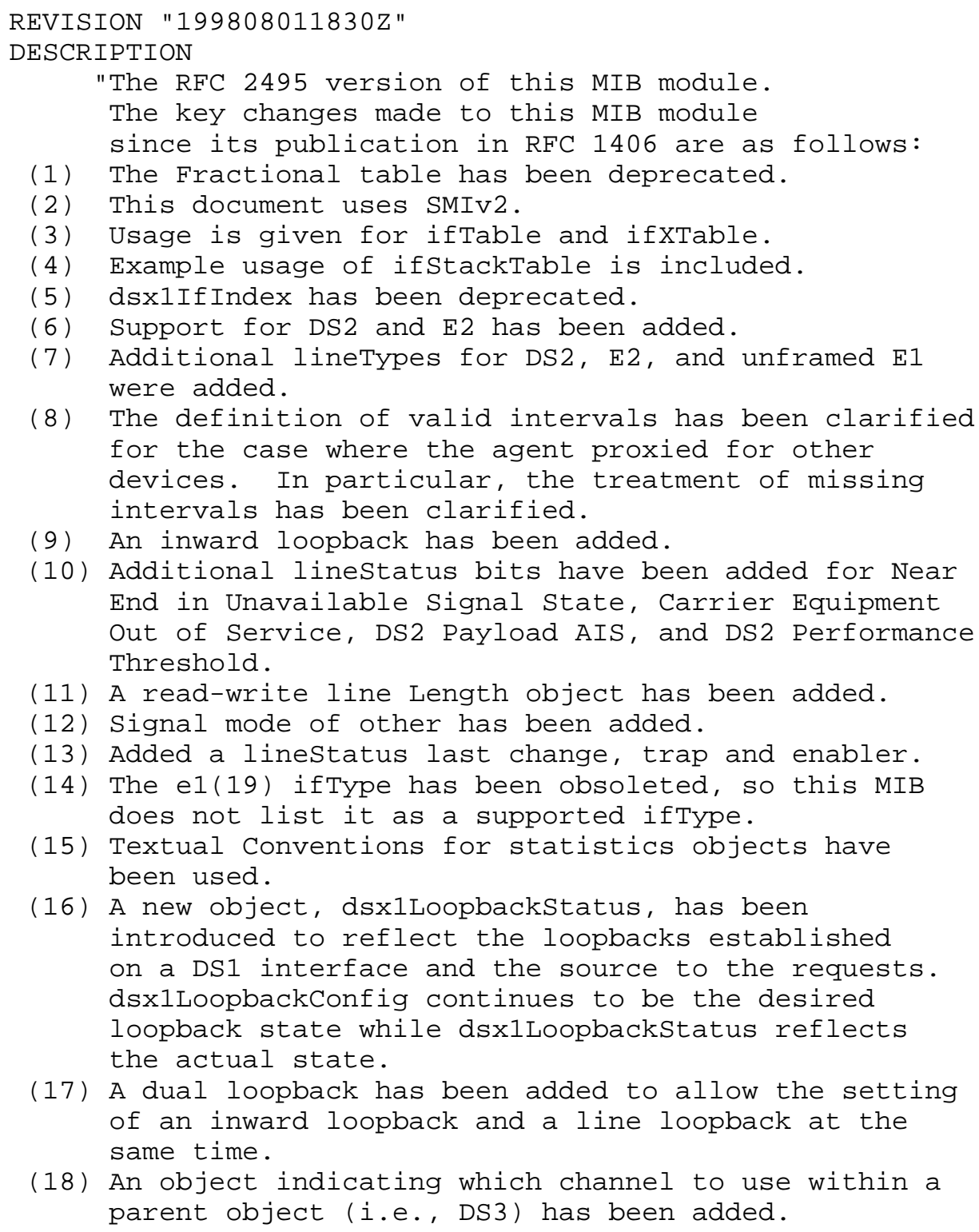

(17) A dual loopback has been added to allow the setting of an inward loopback and a line loopback at the same time.

(18) An object indicating which channel to use within a parent object (i.e., DS3) has been added. 
(19) An object has been added to indicate whether or not this DS1/E1 is channelized.

(20) Line coding type of B6zS has been added for DS2."

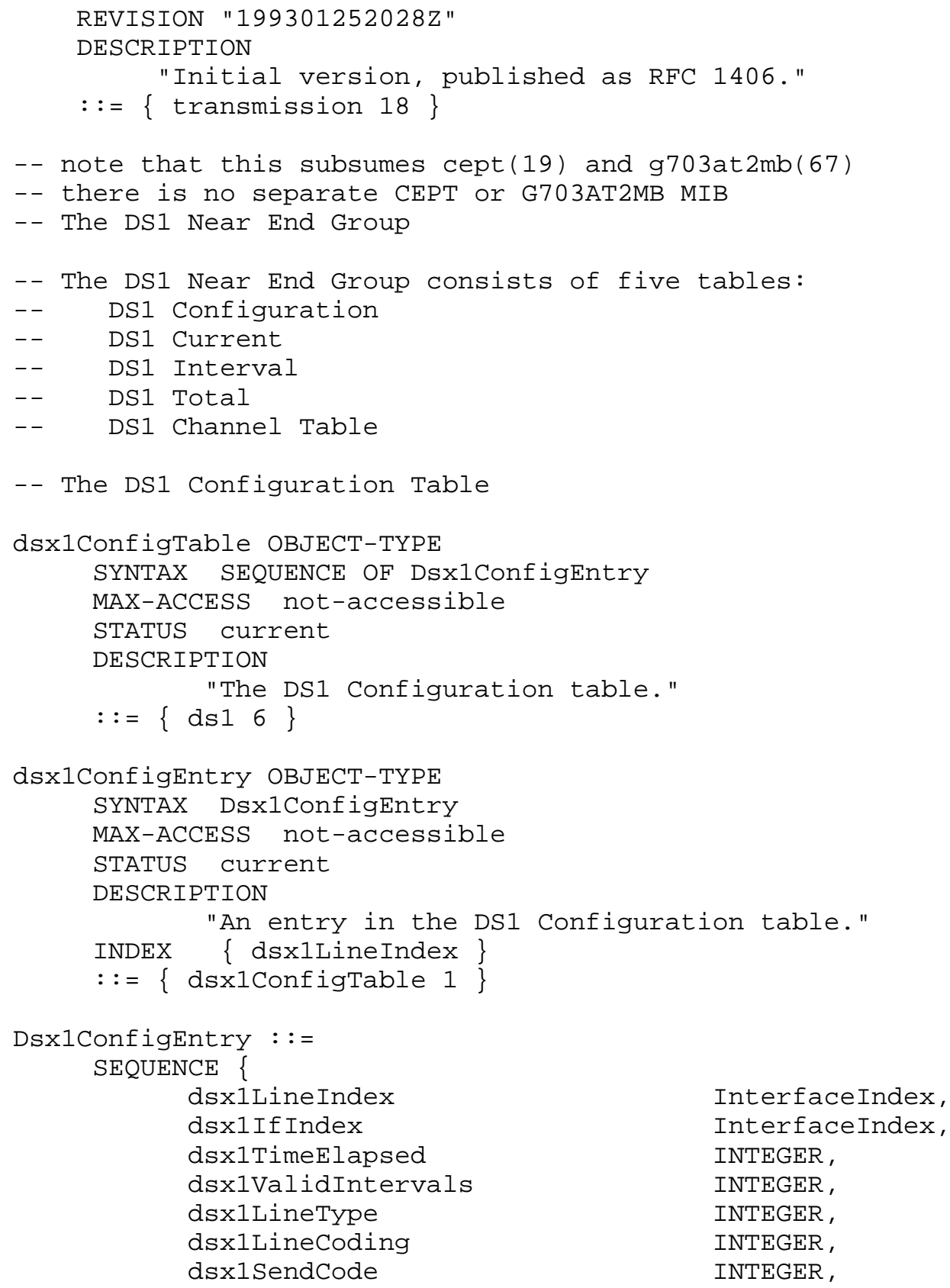




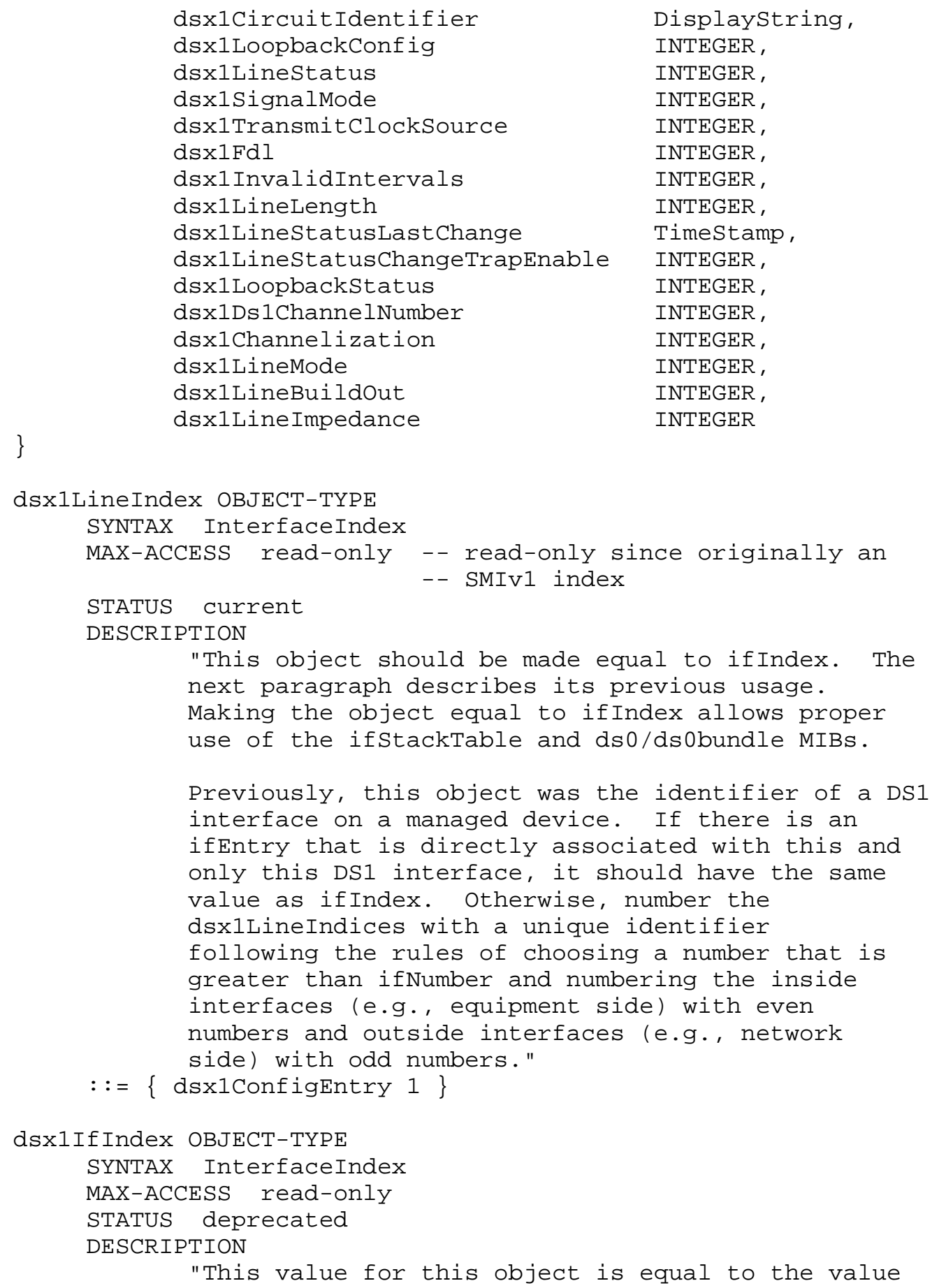




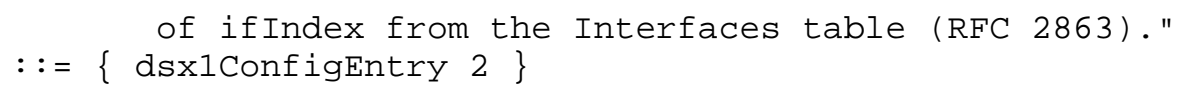




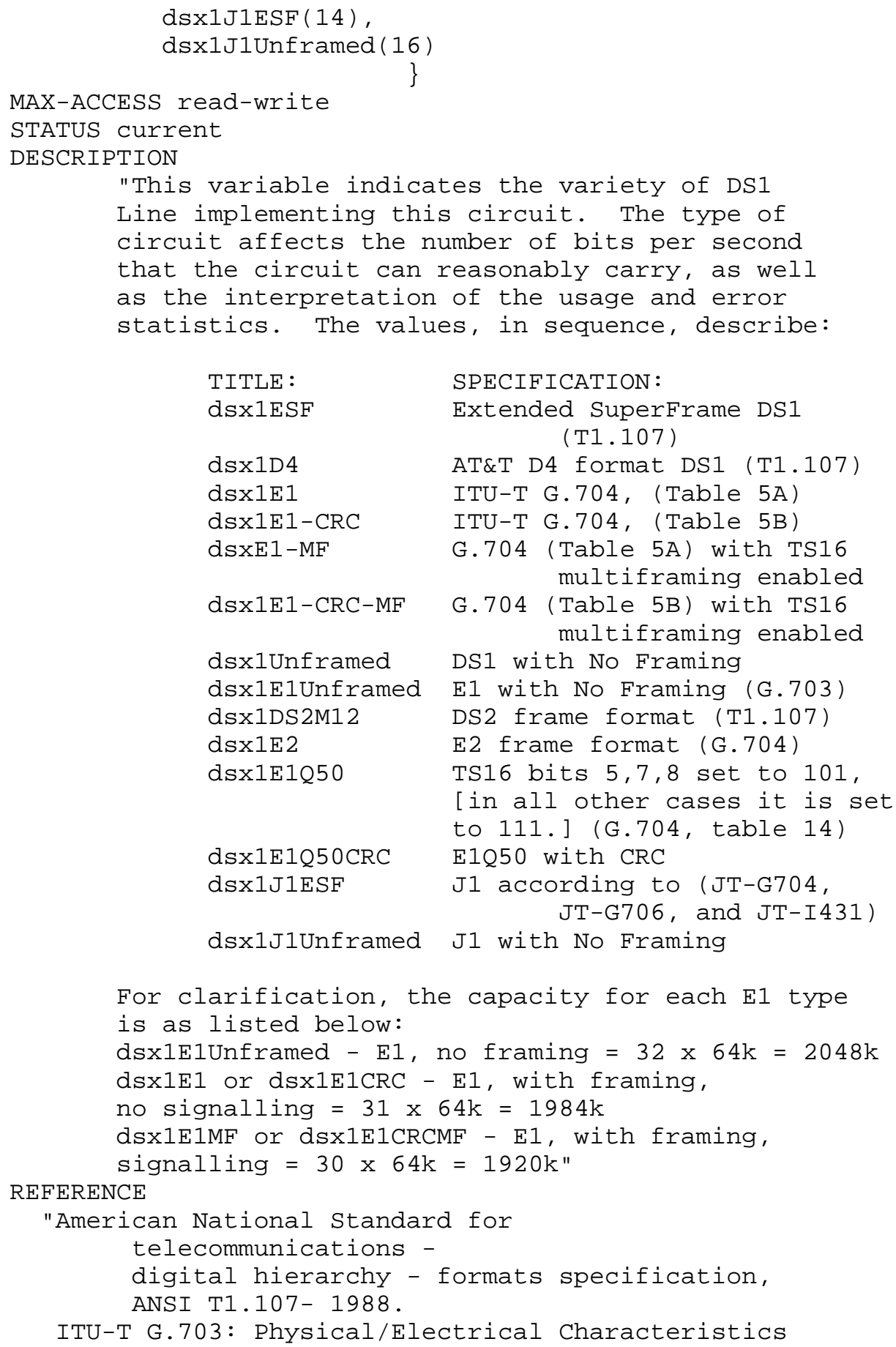




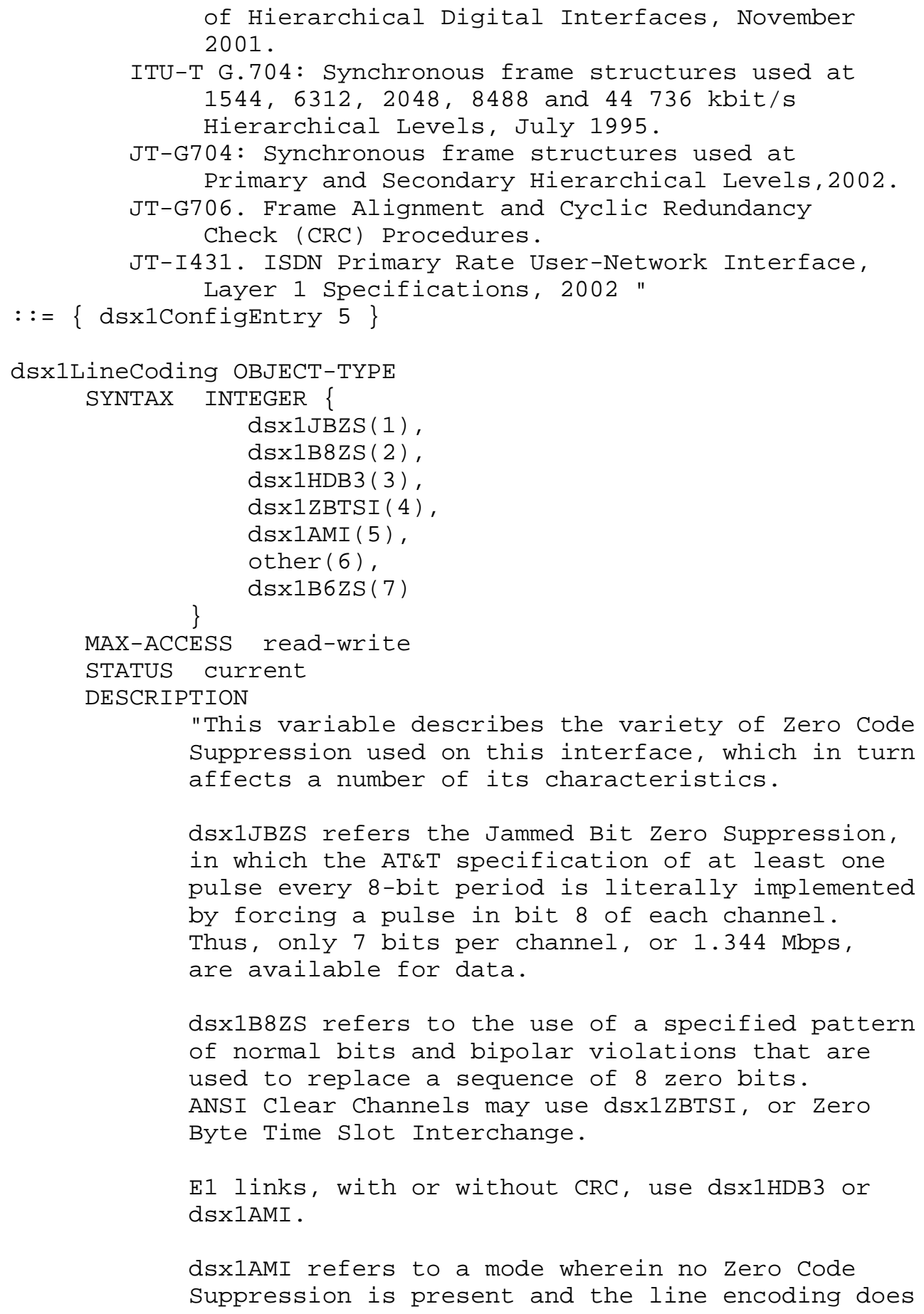




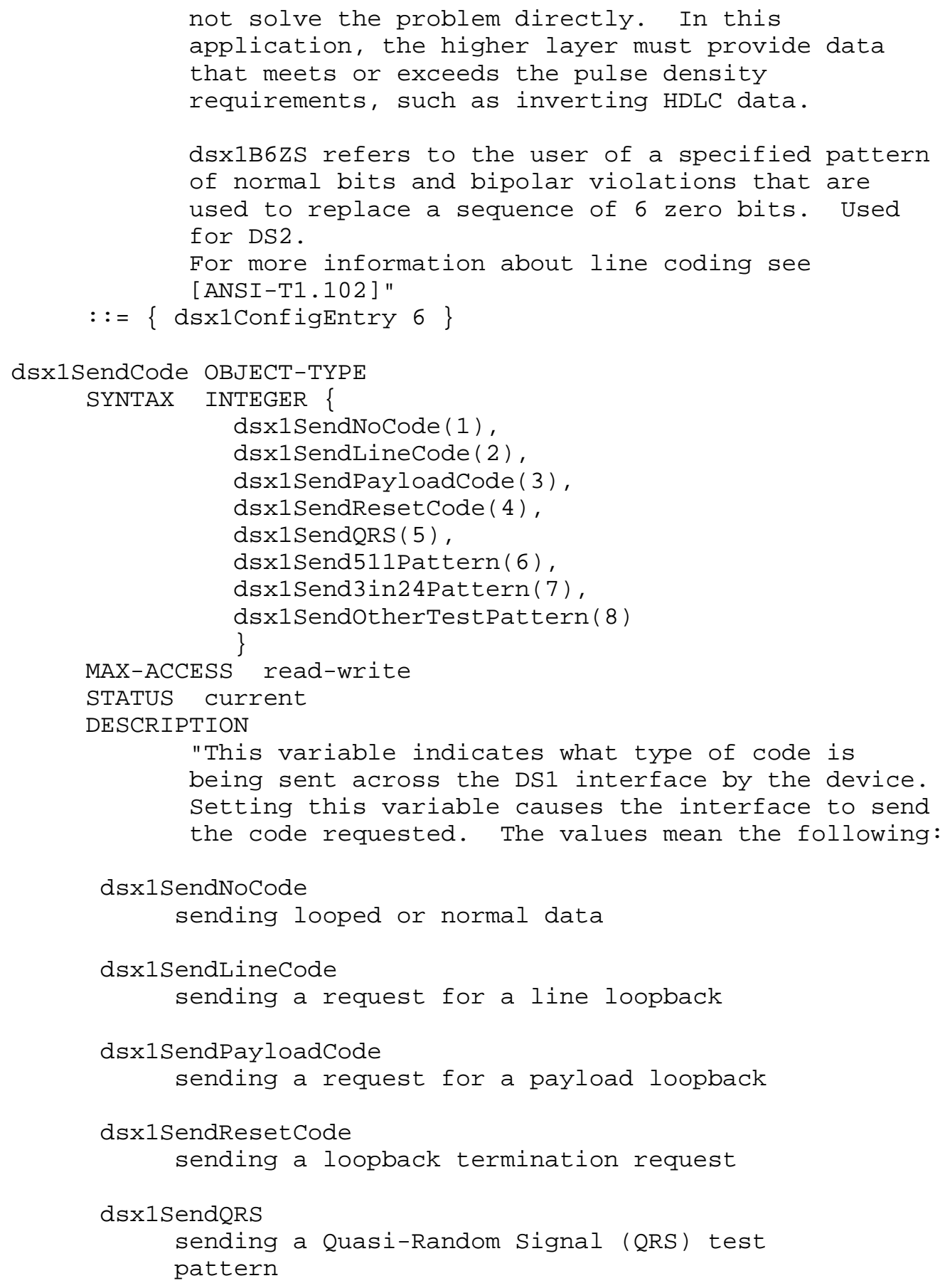




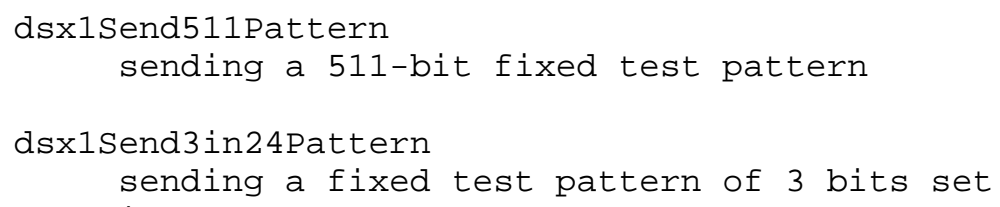


the received signal at this interface is looped

through the device. Typically, the received signal

is looped back for retransmission after it has

passed through the device's framing function.

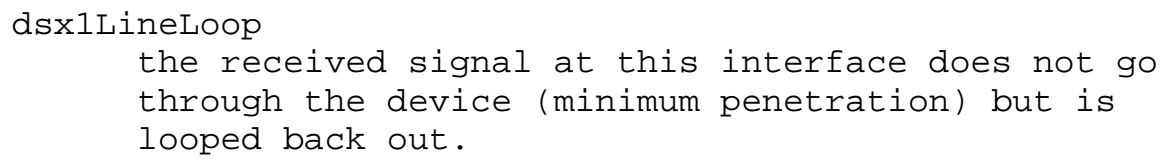




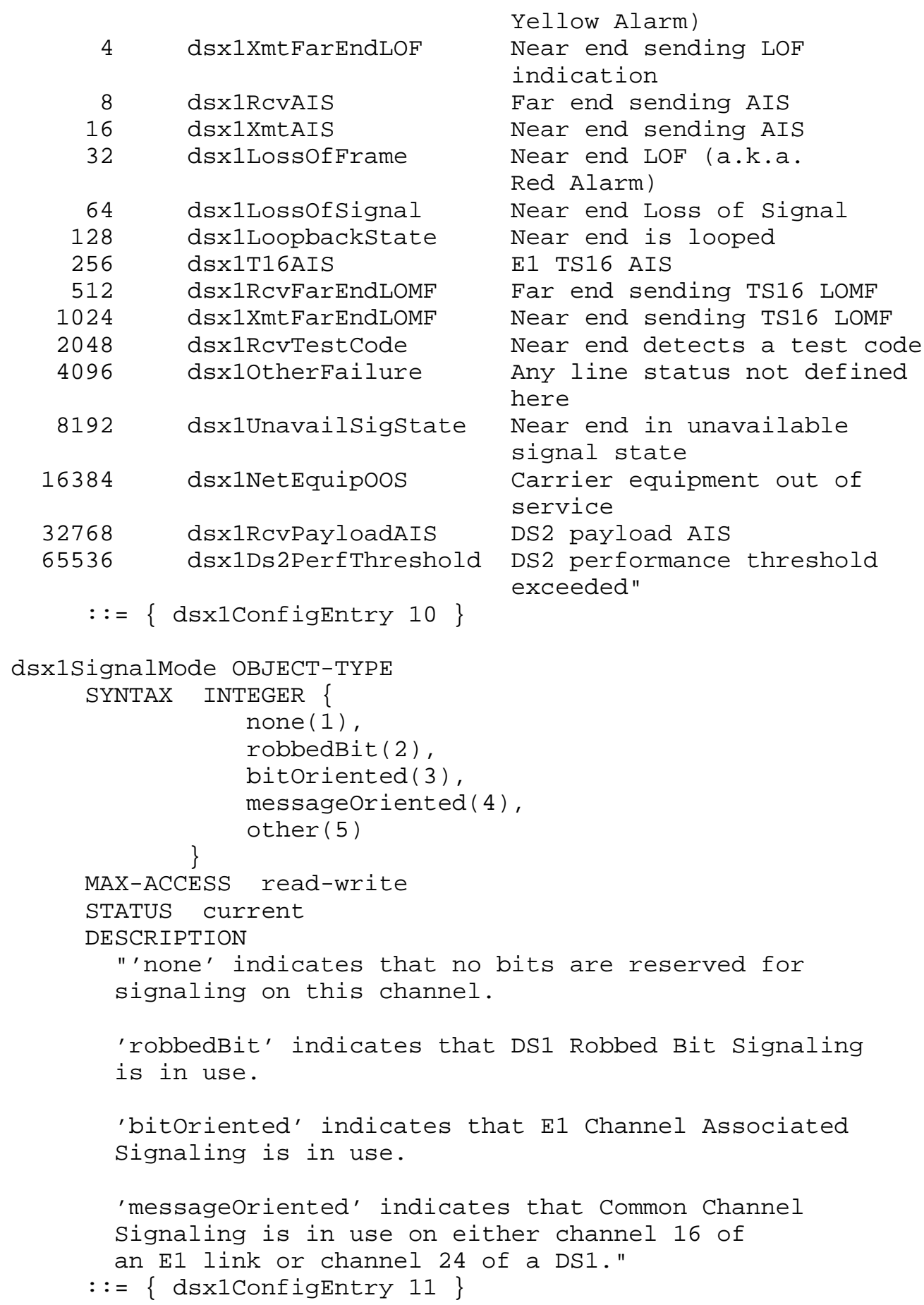




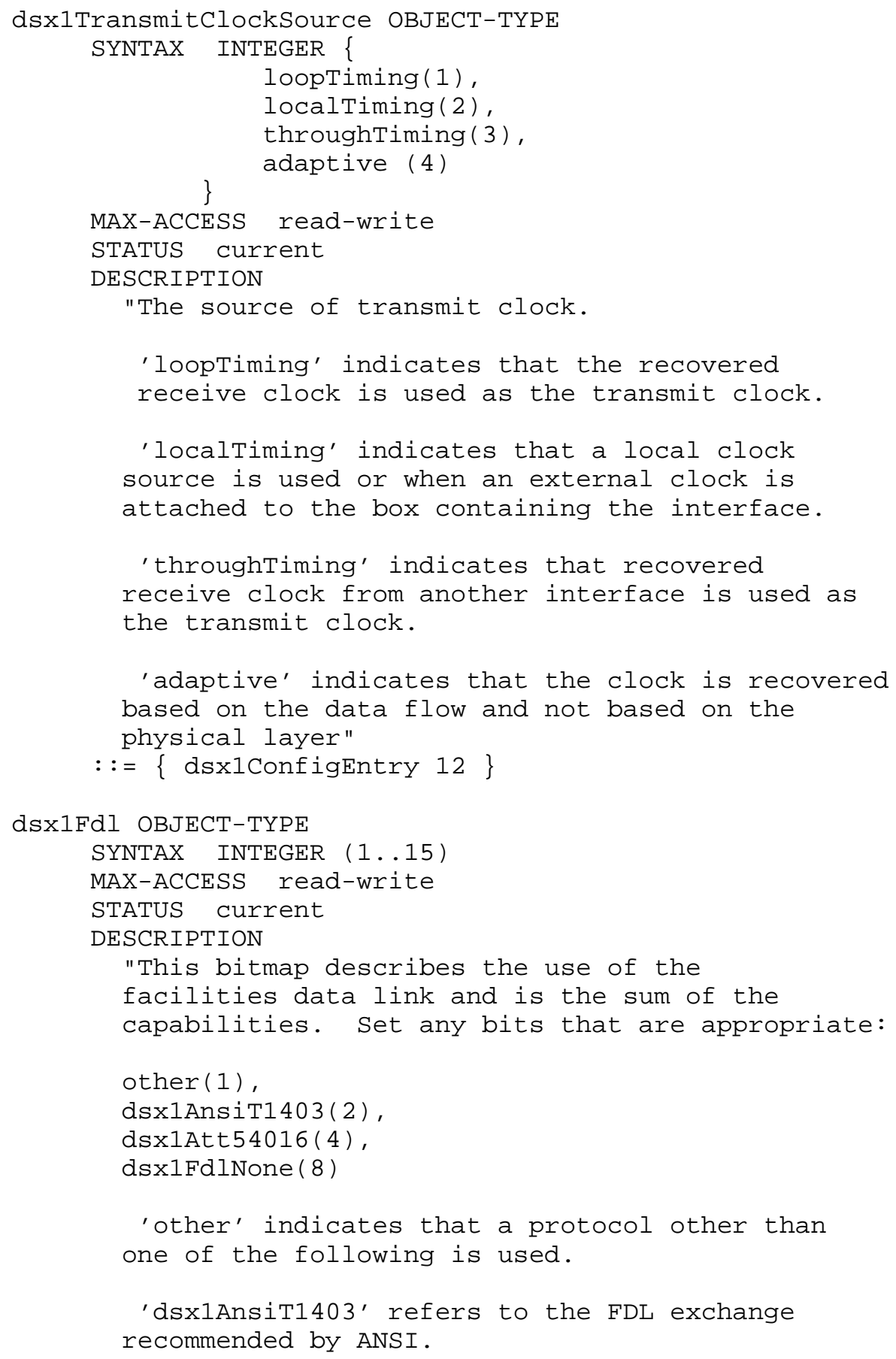




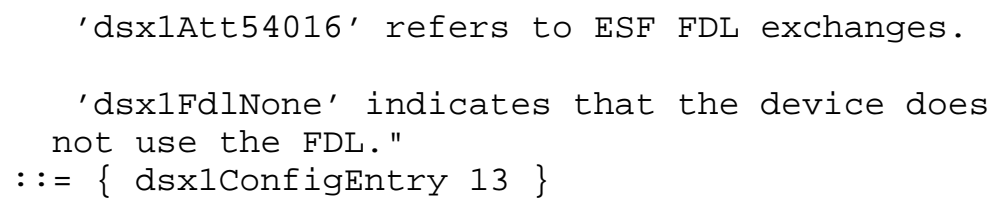




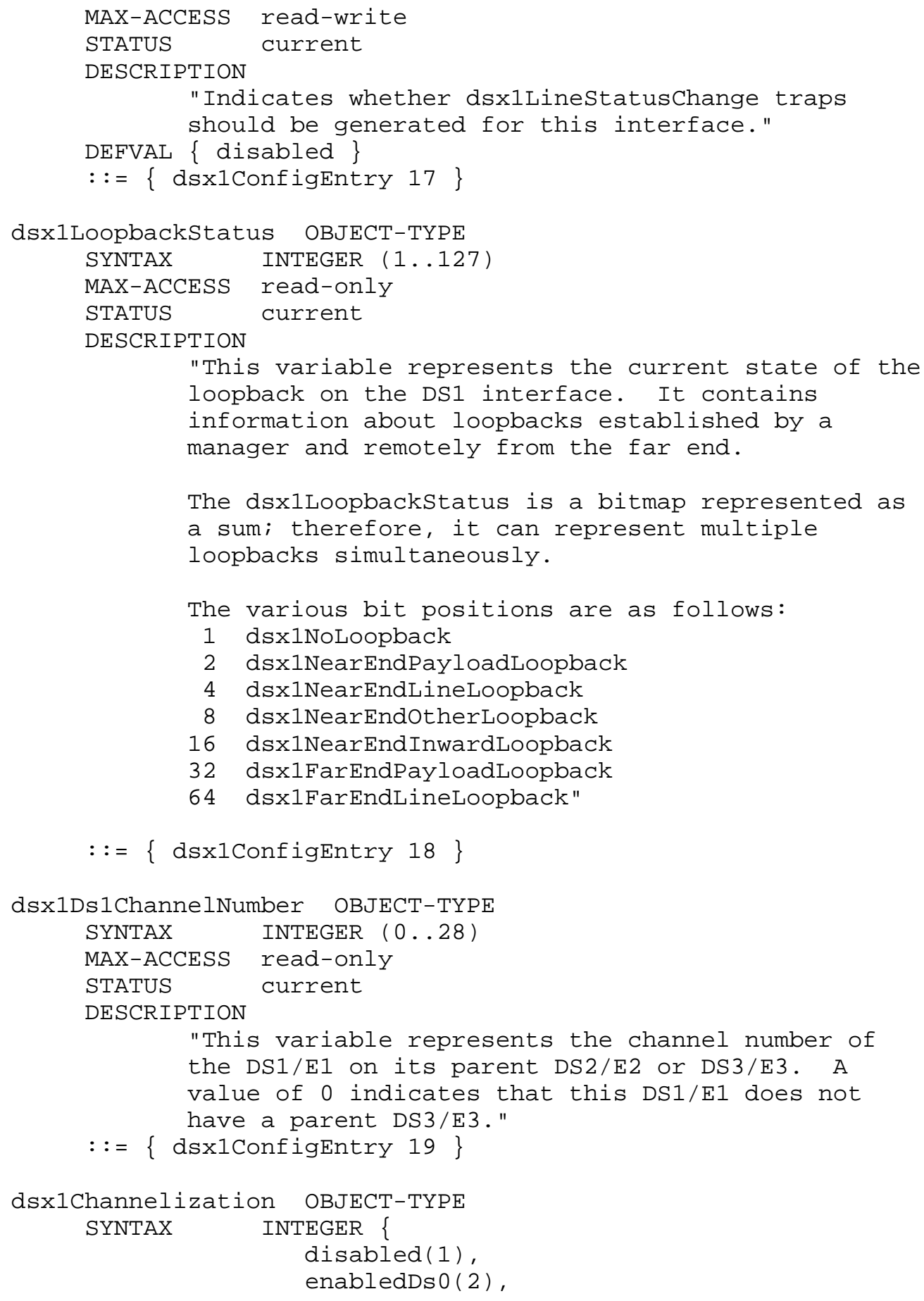




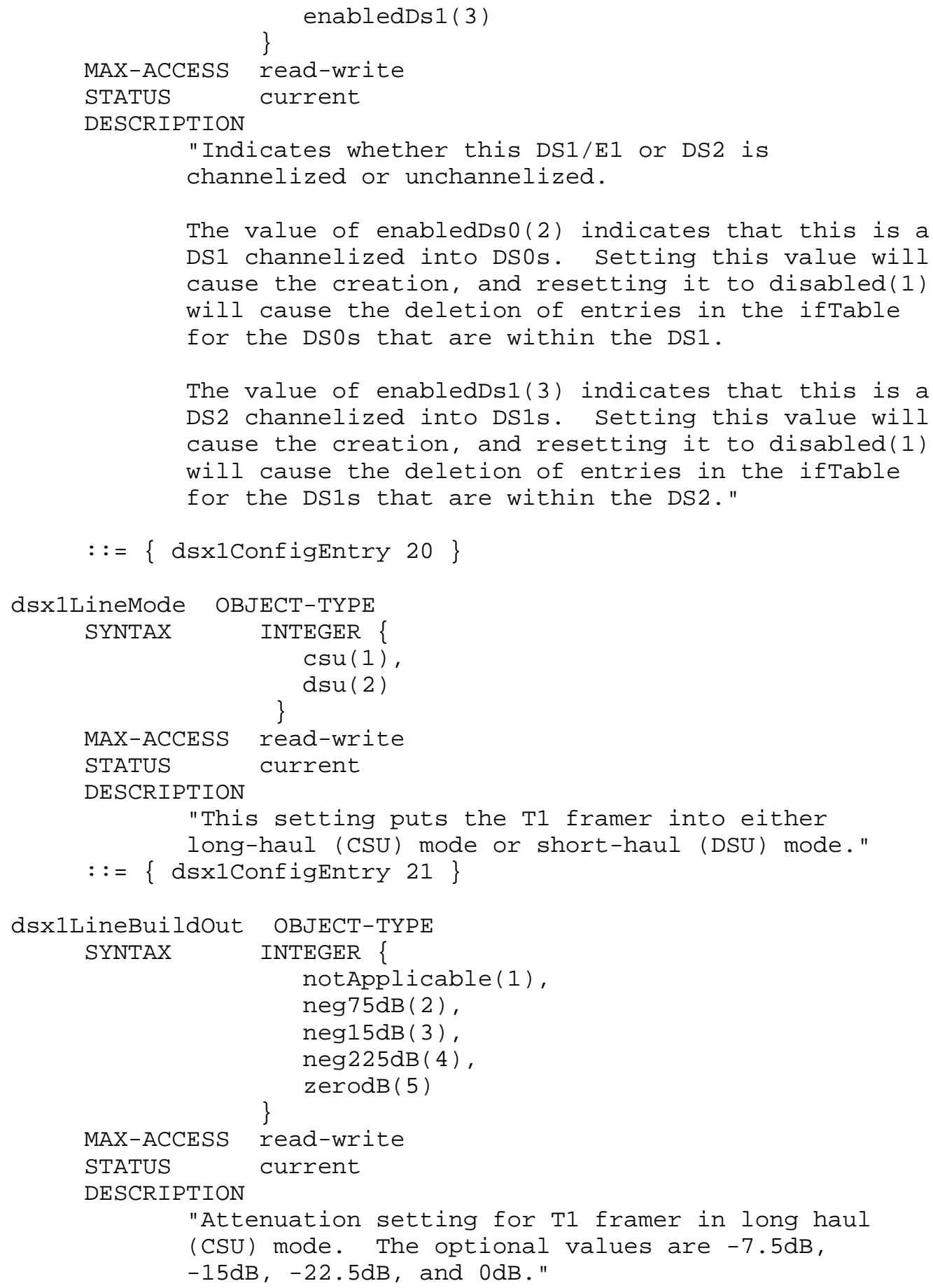


$::=\{$ dsxiConfigEntry 22$\}$

dsX1LineImpedance OBJECT-TYPE

SYNTAX INTEGER \{

notApplicable (1),

unbalanced75ohms (2),

balanced100ohms (3),

\}

balanced120ohms (4)

MAX-ACCESS read-write

STATUS current

DESCRIPTION

"Nominal line impedance. For T1 and J1 lines, the

value is typically balancedlo0ohms(3). For E1

lines, the value is typically unbalanced75ohms (2)

and balanced120ohms(4). When this object does not apply, or when the appropriate value is not known, the value should be set to notApplicable(1)."

$::=\{$ dsxiConfigEntry 23$\}$

-- The DS1 Current Table

dsx1CurrentTable OBJECT-TYPE

SYNTAX SEQUENCE OF DsxlCurrentEntry

MAX-ACCESS not-accessible

STATUS current

DESCRIPTION

"The DS1 Current table contains various statistics

being collected for the current 15-minute

interval."

$::=\{\operatorname{ds} 17\}$

dsx1CurrentEntry OBJECT-TYPE

SYNTAX DsX1CurrentEntry

MAX-ACCESS not-accessible

STATUS current

DESCRIPTION

"An entry in the DS1 Current table."

INDEX \{ dsxicurrentIndex $\}$

$::=\{$ dsxicurrentTable 1$\}$

DsxicurrentEntry : :=

SEQUENCE \{

dsxicurrentIndex InterfaceIndex,

dsxicurrentESs

dsx1CurrentSESs

PerfCurrentCount,

dsx1CurrentSEFSs PerfCurrentCount,

dsx1CurrentUASs

PerfCurrentCount,

dsx1CurrentCSSs

PerfCurrentCount,

PerfCurrentCount, 


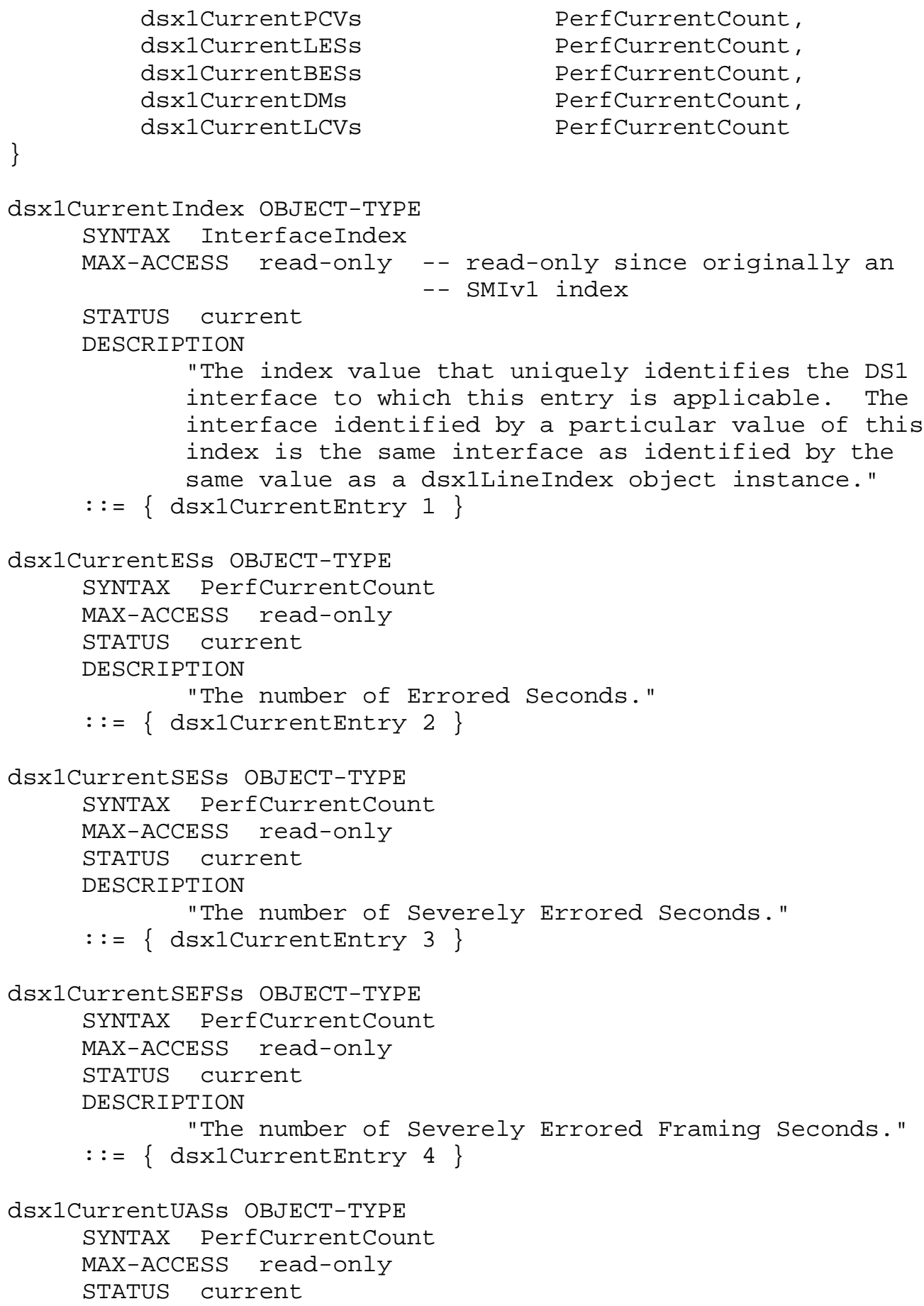




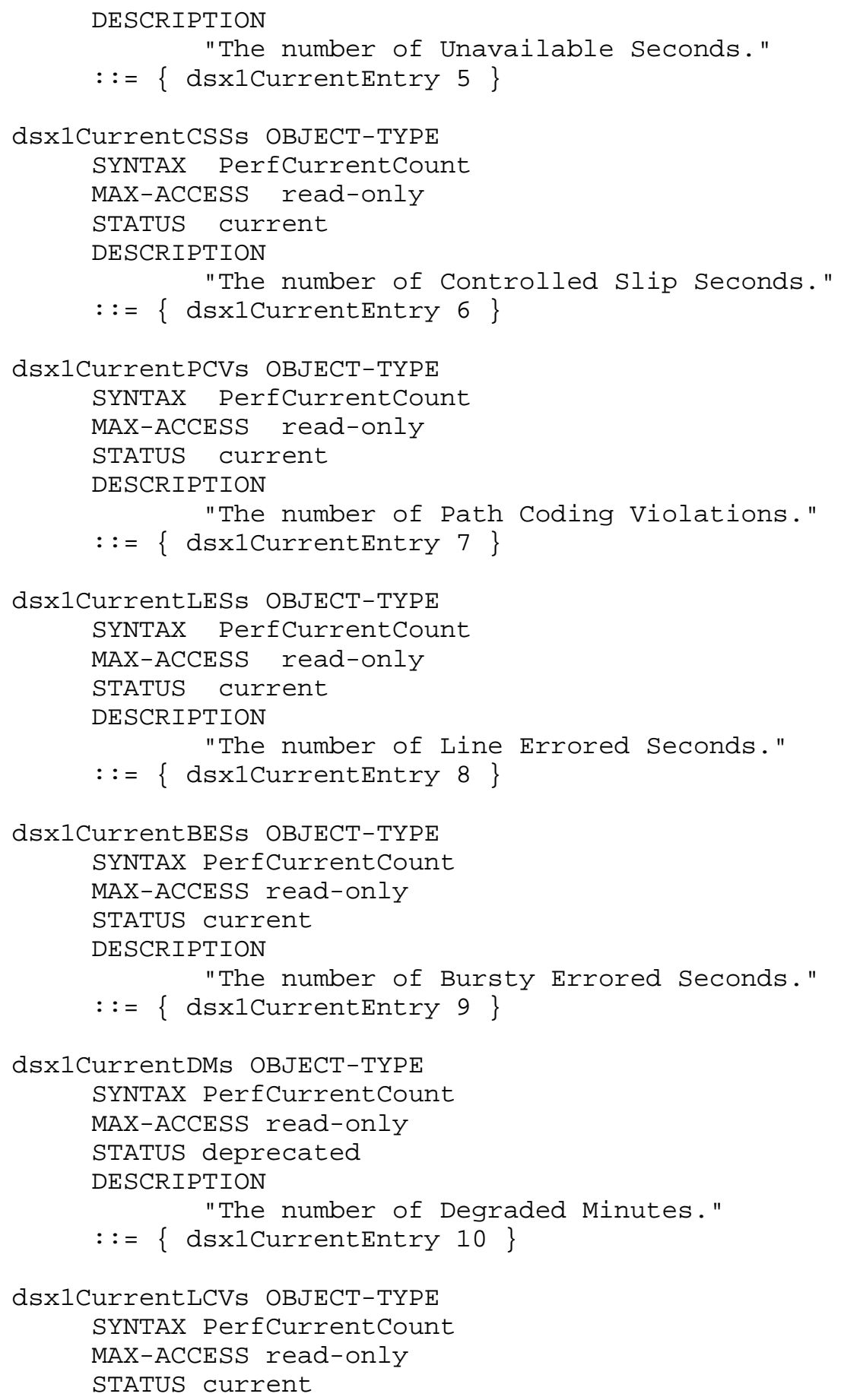




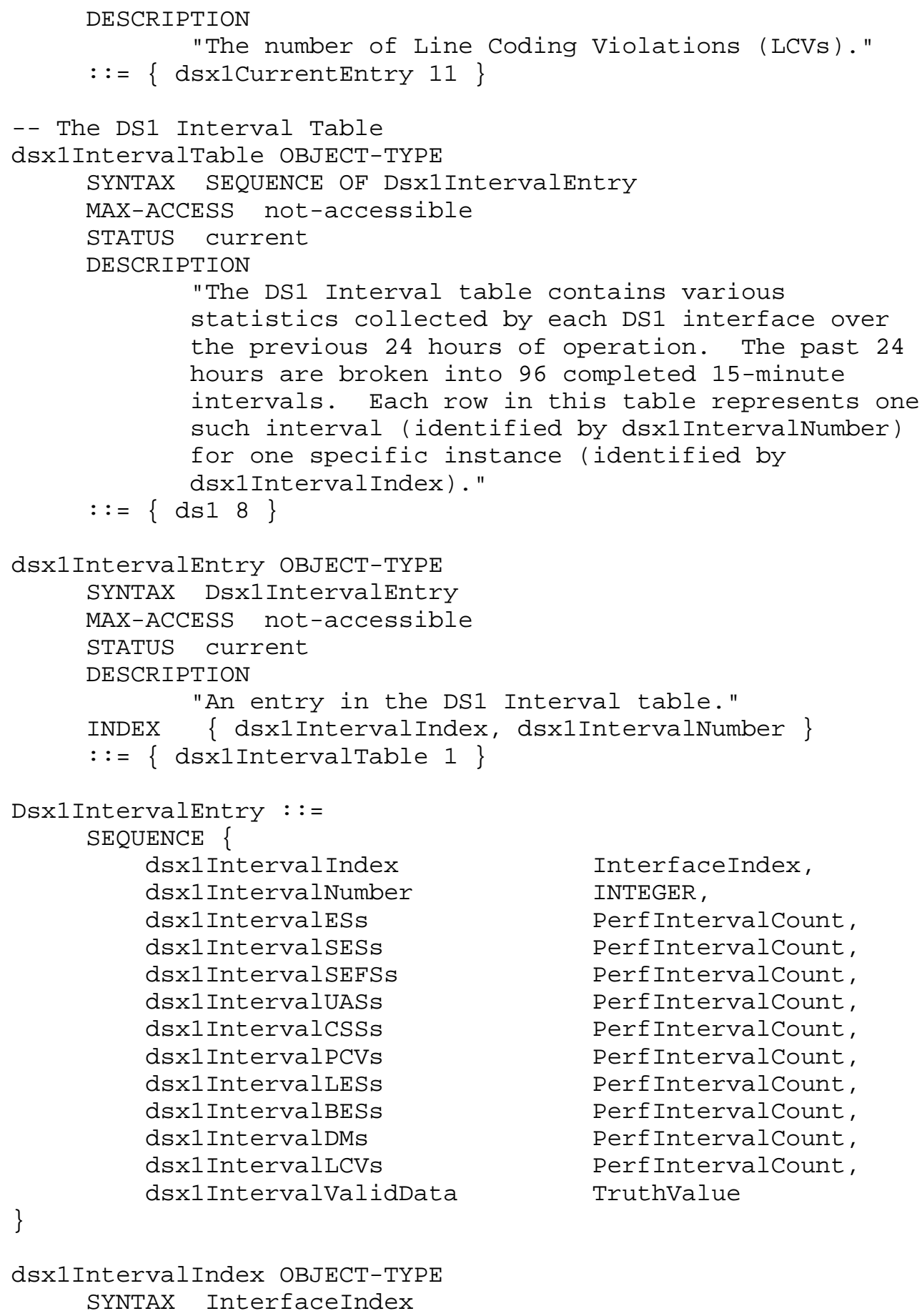




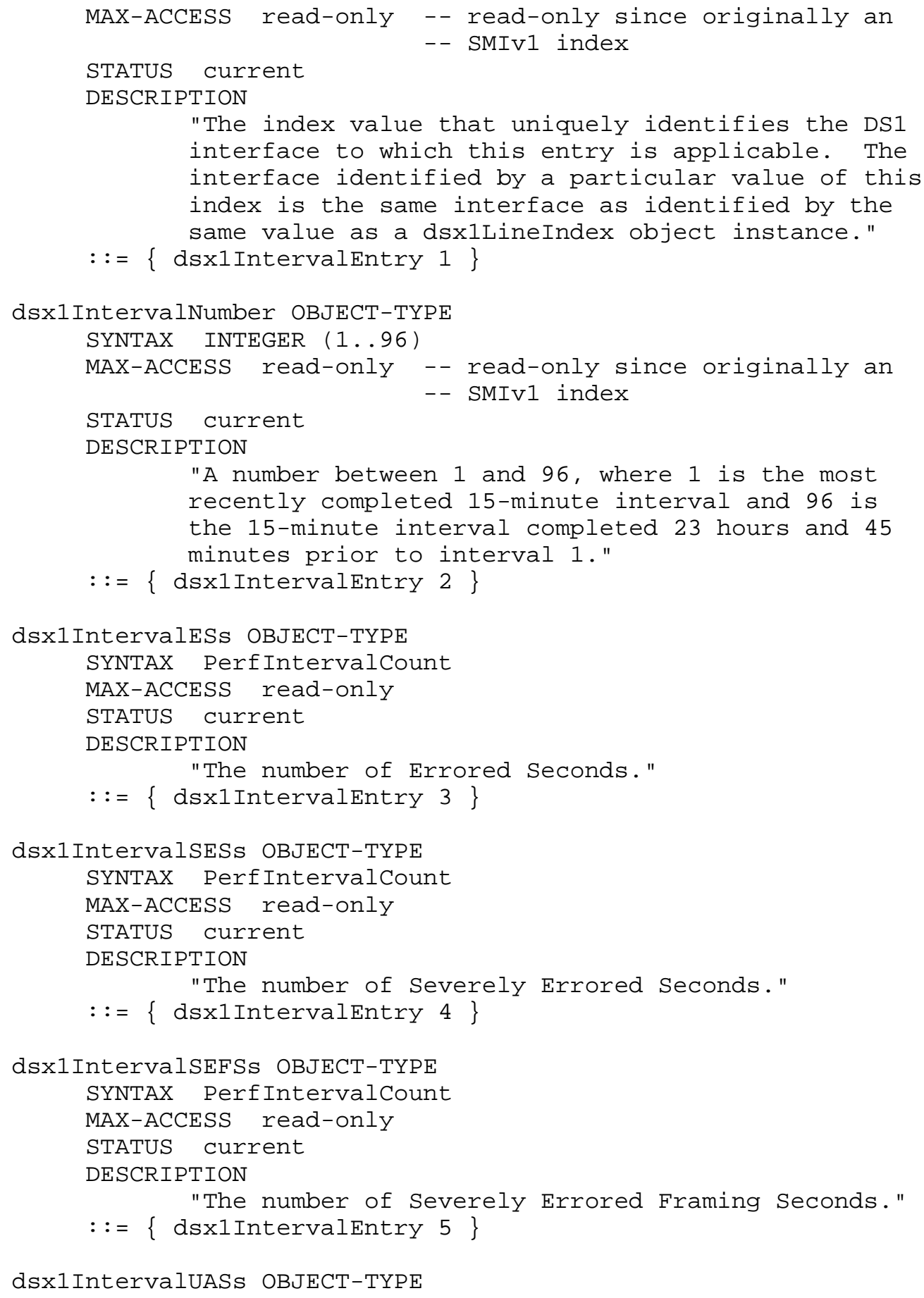




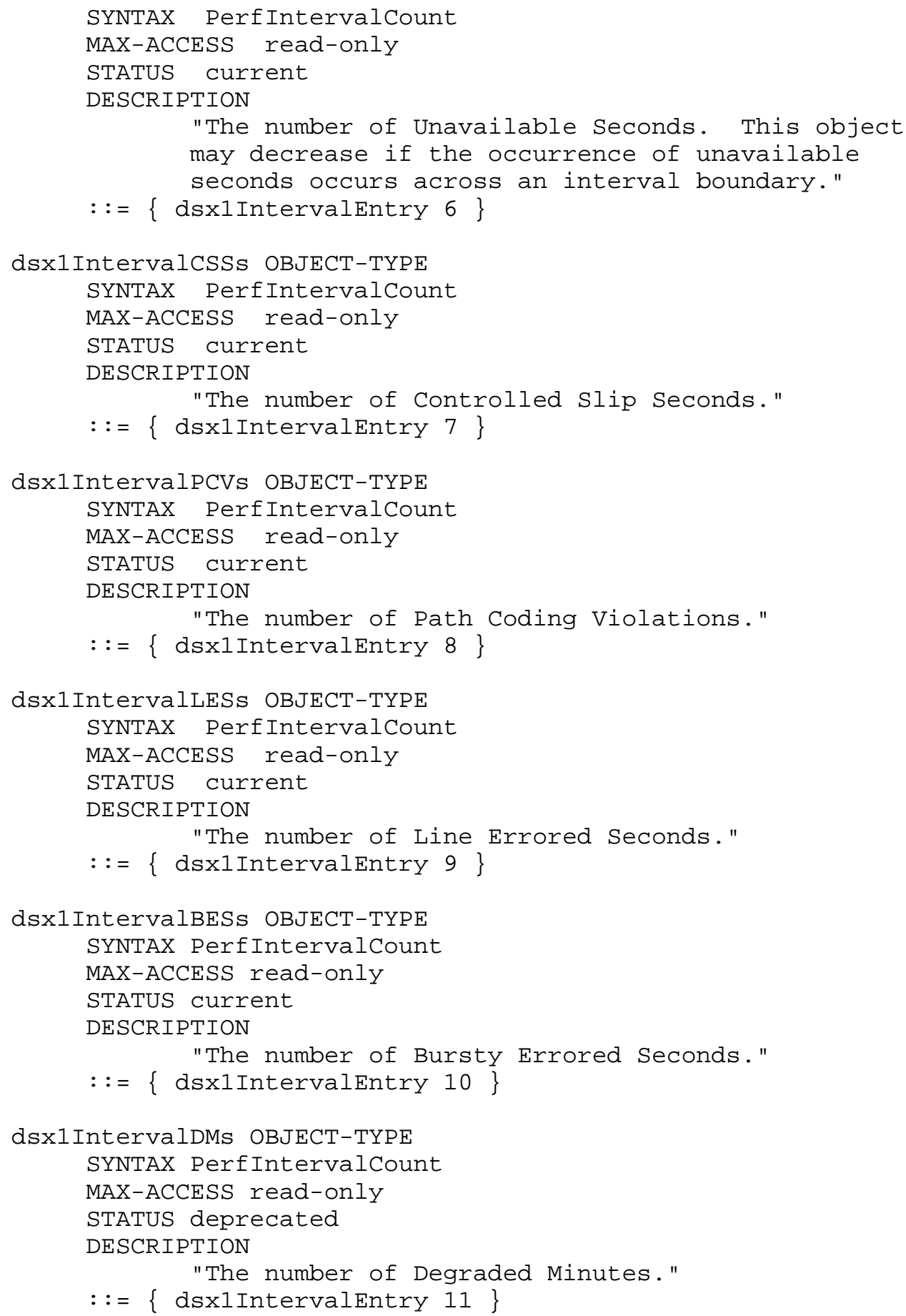




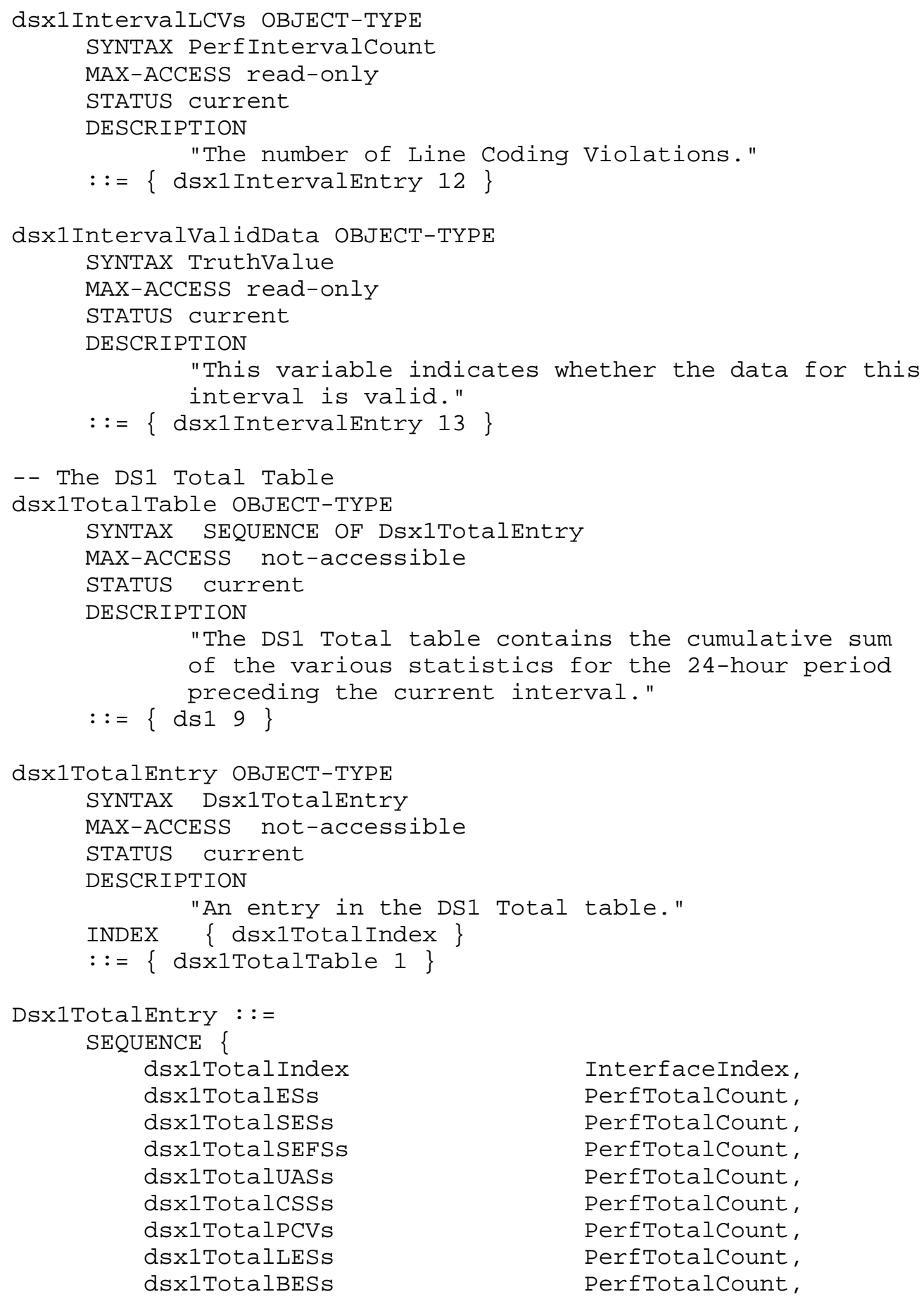

Nicklass, Ed. Standards Track [Page 42] 


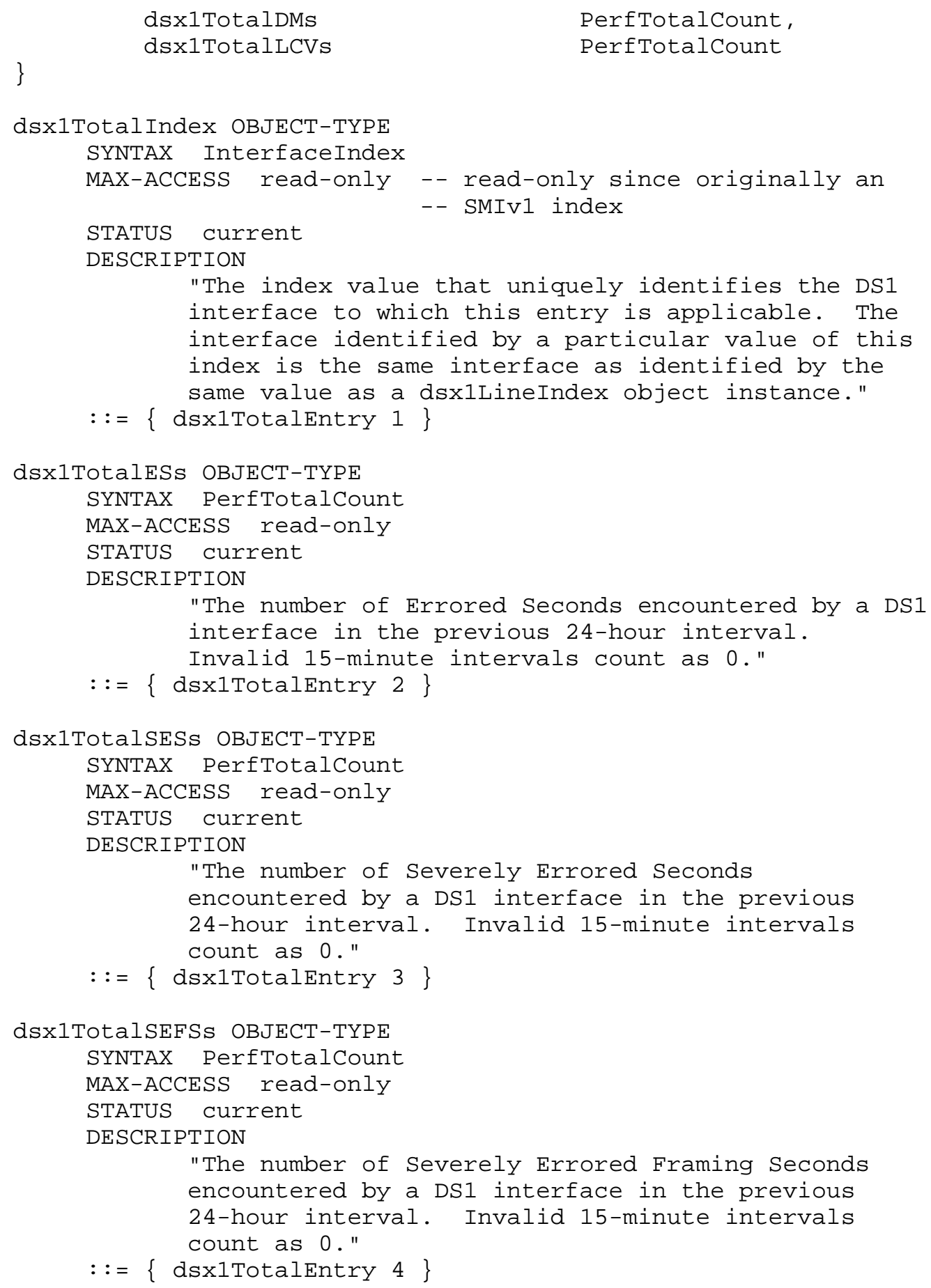




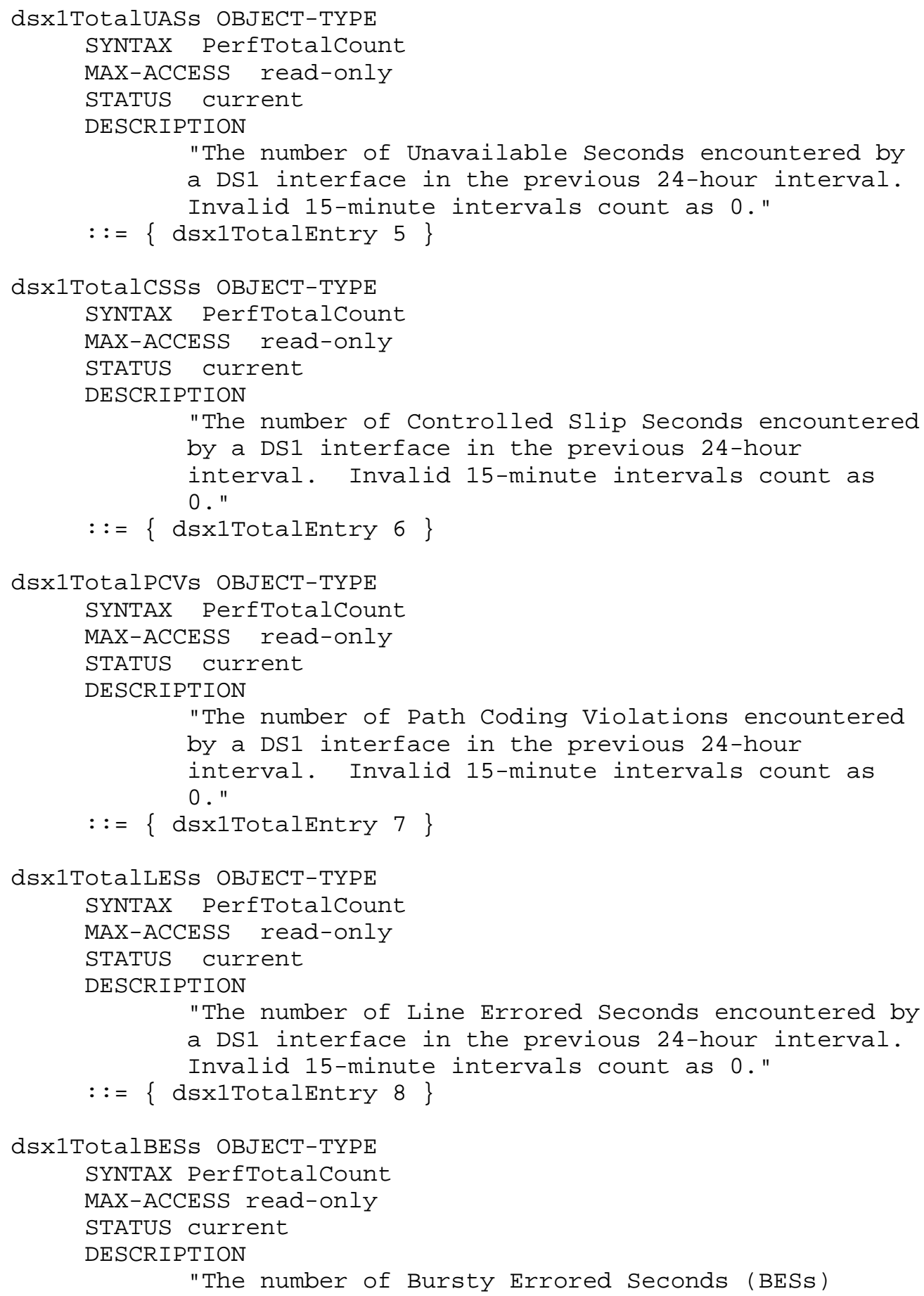




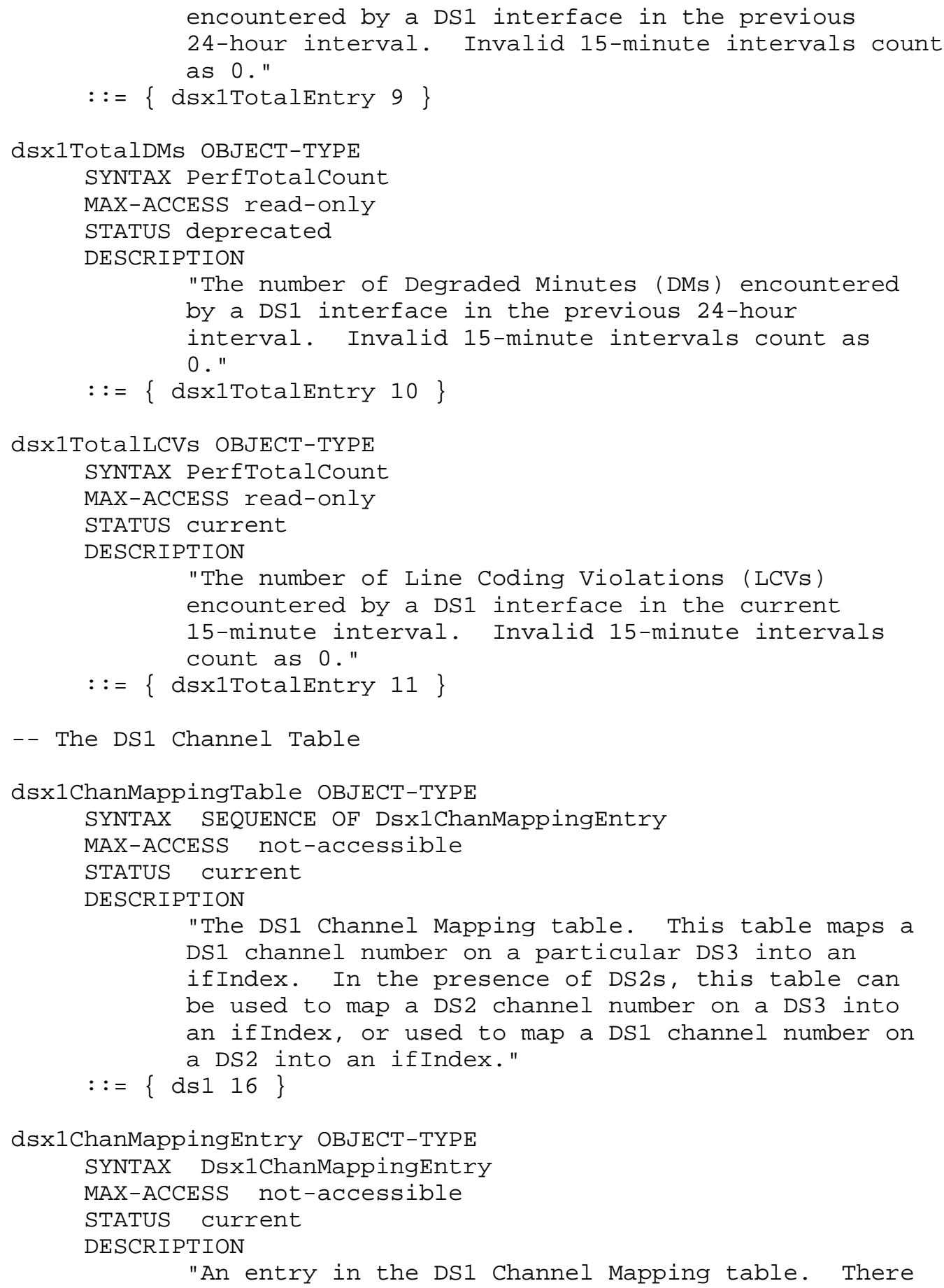




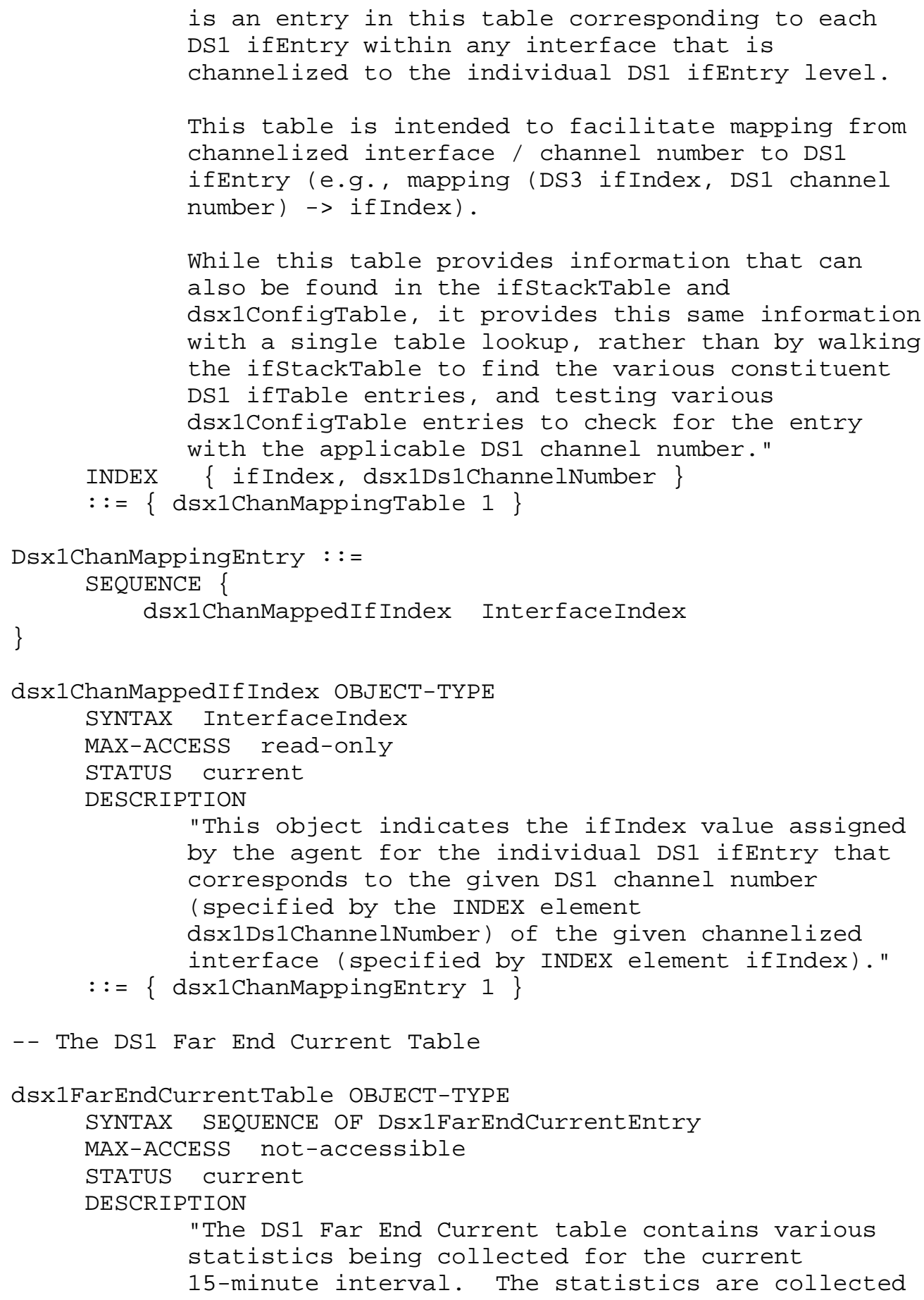


from the far-end messages on the Facilities Data Link. The definitions are the same as described for the near-end information." $::=\{\mathrm{ds} 110\}$

dsX1FarEndCurrentEntry OBJECT-TYPE SYNTAX DsX1FarEndCurrentEntry MAX-ACCESS not-accessible

STATUS current DESCRIPTION

"An entry in the DS1 Far End Current table." INDEX \{dsxiFarEndCurrentIndex $\}$ $::=\{$ dsxifarEndCurrentTable 1$\}$

Dsx1FarEndCurrentEntry : := SEQUENCE \{ dsxifarEndCurrentIndex InterfaceIndex, dsxifarEndTimeElapsed INTEGER, dsxlFarEndValidIntervals INTEGER, dsxifarEndCurrentESs dsx1FarEndCurrentSESs dsx1FarEndCurrentSEFSs dsx1FarEndCurrentUASs dsx1FarEndCurrentCSSs PerfCurrentCount, PerfCurrentCount, PerfCurrentCount, PerfCurrentCount, PerfCurrentCount, dsx1FarEndCurrentLESs PerfCurrentCount, dsx1FarEndCurrentPCVs PerfCurrentCount, dsx1FarEndCurrentBESs PerfCurrentCount, dsx1FarEndCurrentDMs PerfCurrentCount, \} dsxifarEndInvalidIntervals INTEGER

dsx1FarEndCurrentIndex OBJECT-TYPE SYNTAX InterfaceIndex MAX-ACCESS read-only -- read-only since originally an -- SMIv1 index

STATUS current DESCRIPTION

"The index value that uniquely identifies the DS1 interface to which this entry is applicable. The interface identified by a particular value of this index is identical to the interface identified by the same value of dsxlLineIndex." $::=\{$ dsxifarEndCurrentEntry 1$\}$

dsX1FarEndTimeElapsed OBJECT-TYPE

SYNTAX INTEGER (0..899)

MAX-ACCESS read-only

STATUS current 


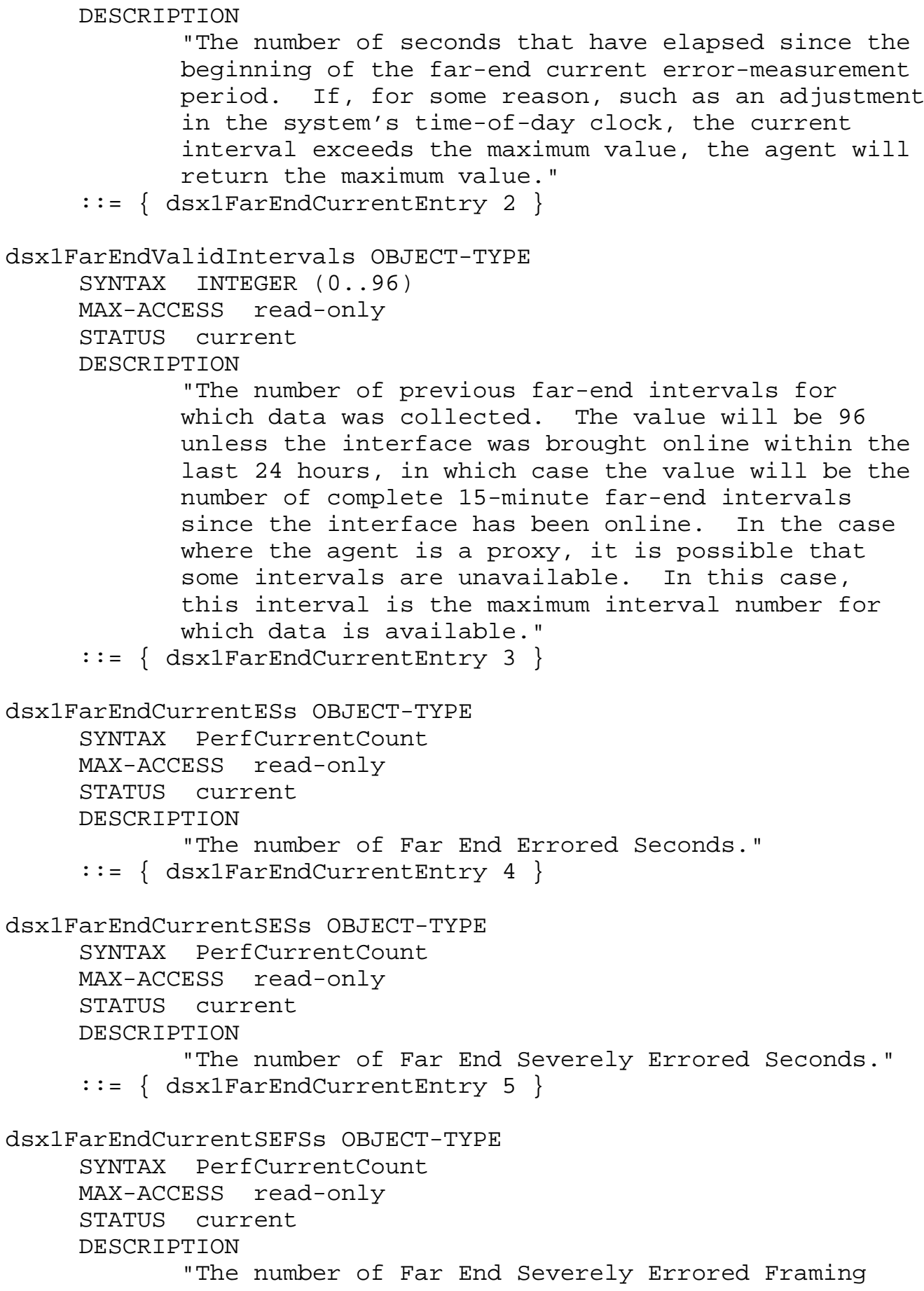




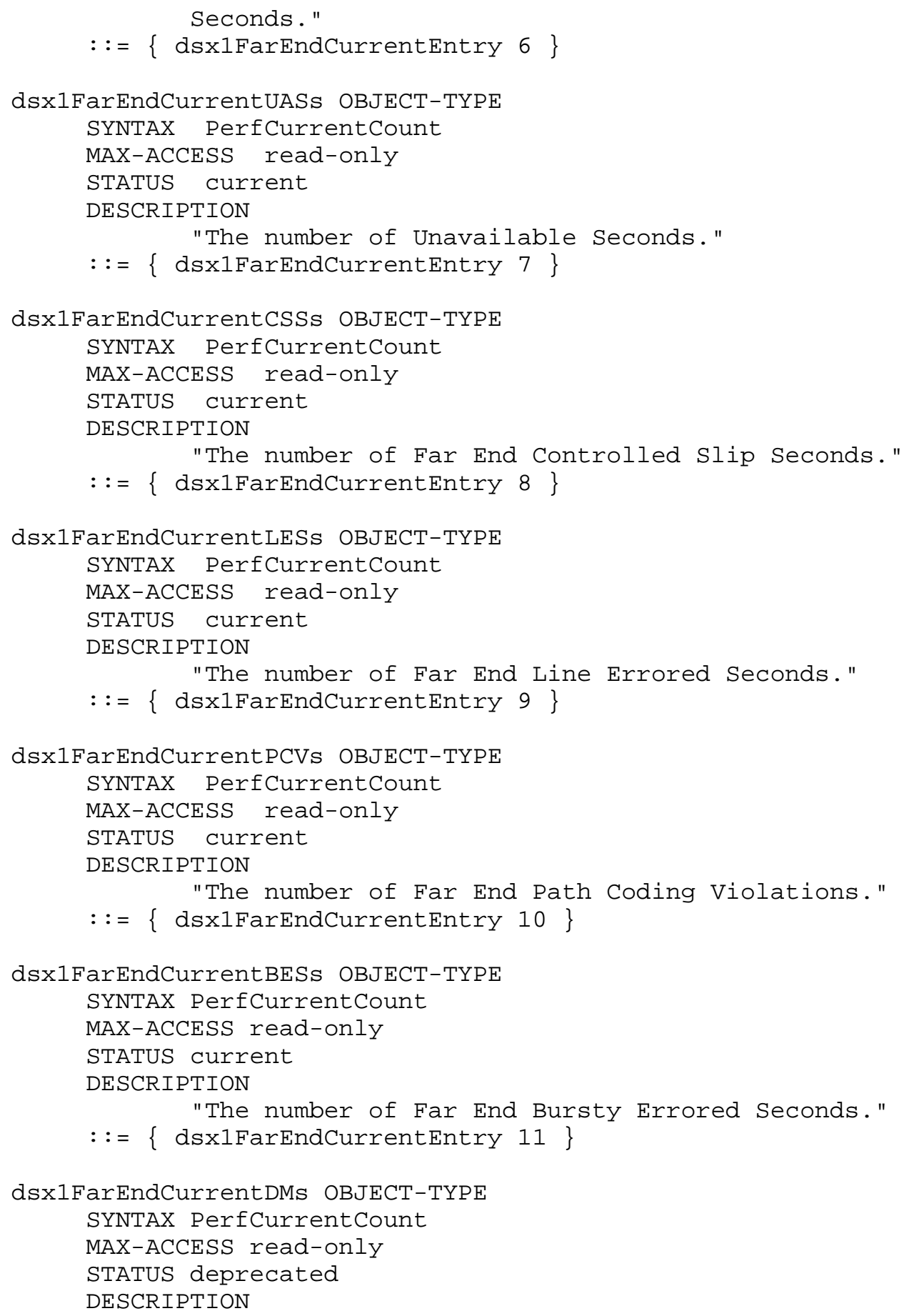




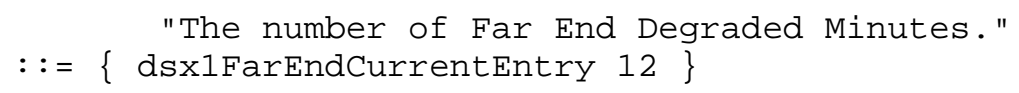




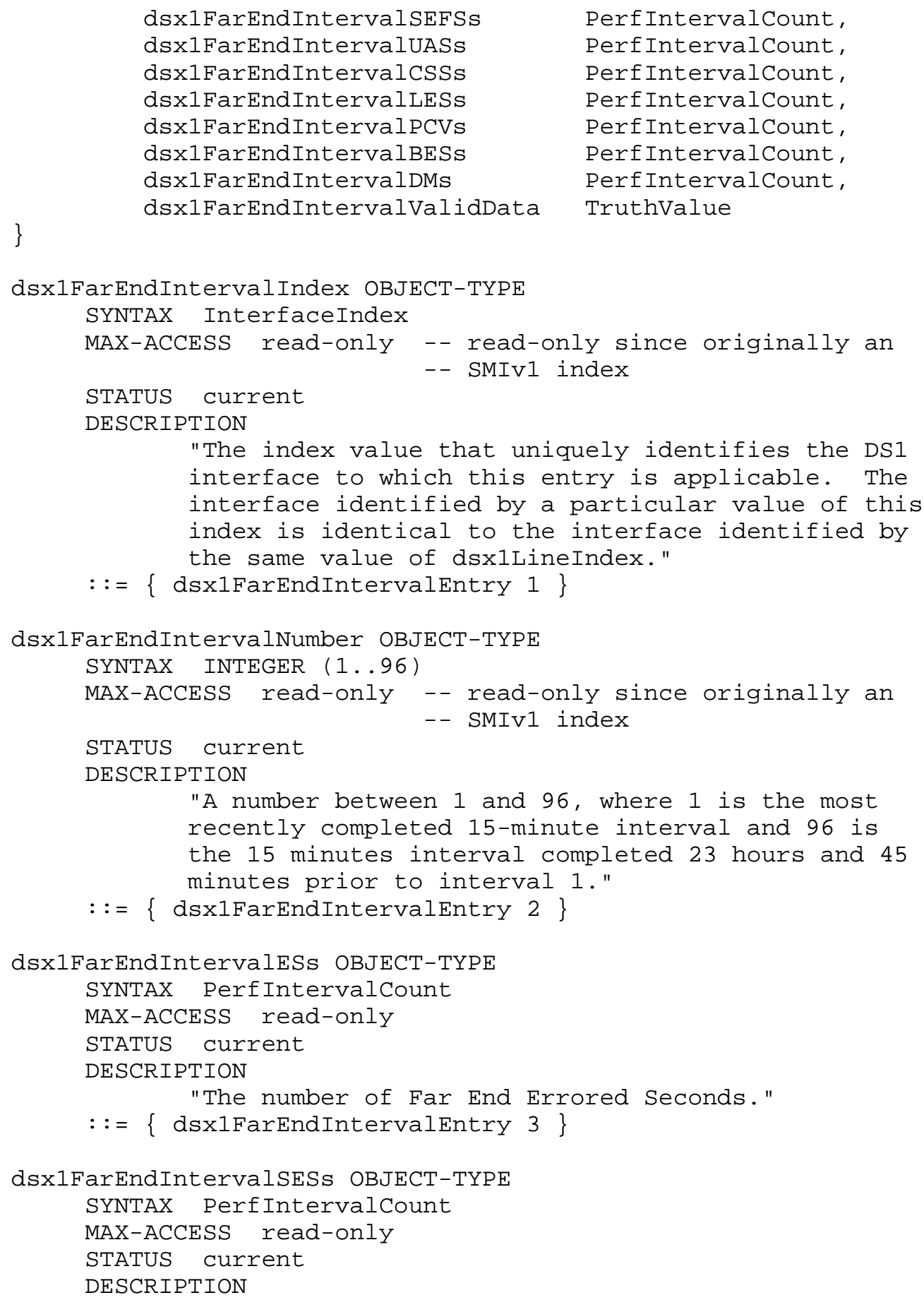




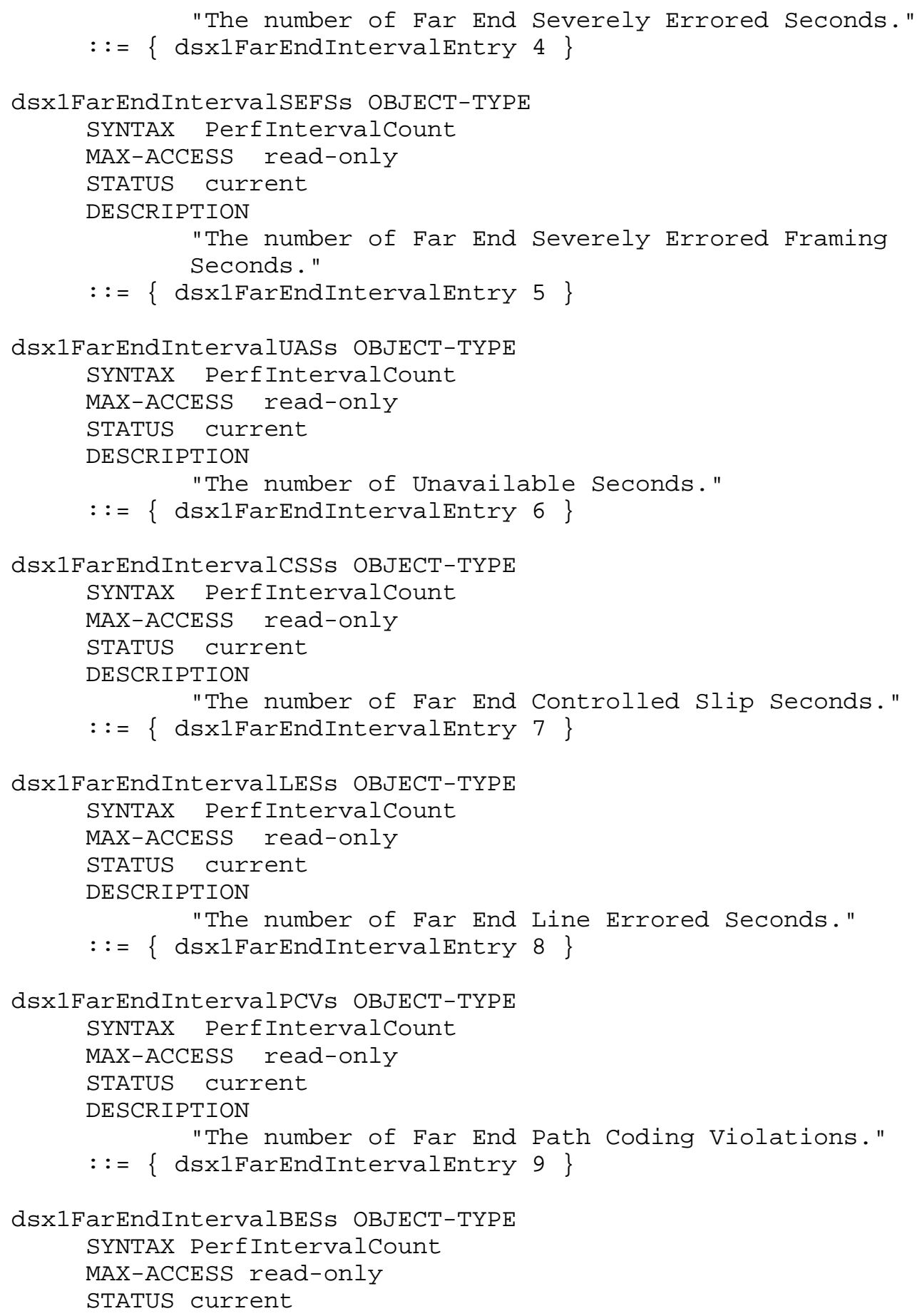




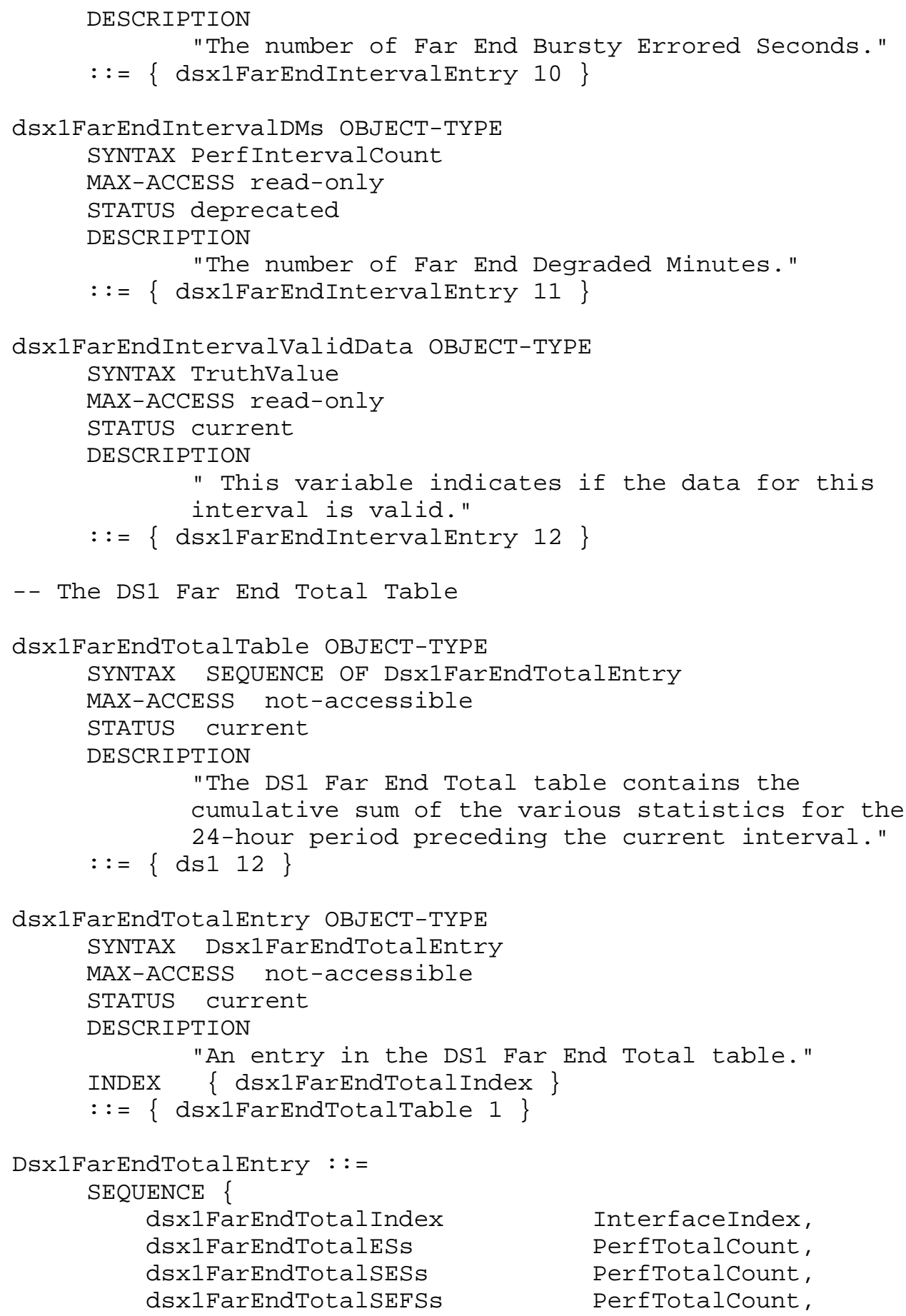




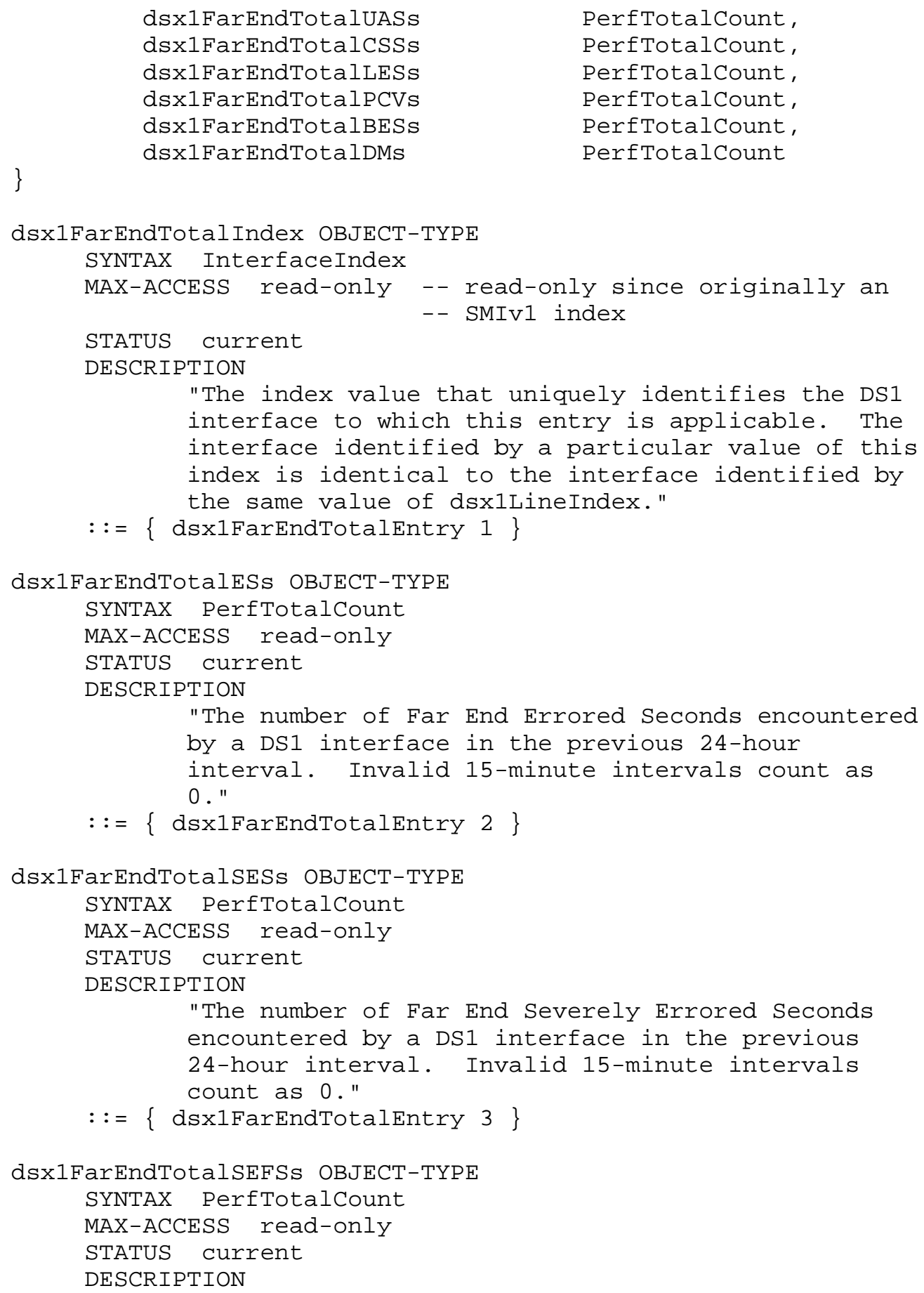




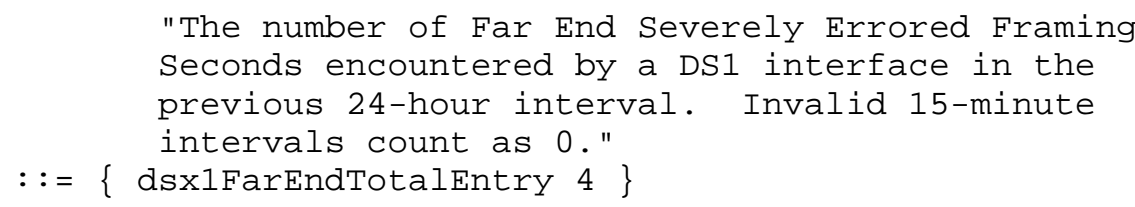


$::=\{$ dsxifarEndTotalEntry 8$\}$

dsX1FarEndTotalBESs OBJECT-TYPE

SYNTAX PerfTotalcount

MAX-ACCESS read-only

STATUS current

DESCRIPTION

"The number of Bursty Errored Seconds (BESS)

encountered by a DS1 interface in the previous

24-hour interval. Invalid 15-minute intervals count as $0 . "$

$::=\{$ dsxifarEndTotalEntry 9$\}$

dsX1FarEndTotalDMs OBJECT-TYPE

SYNTAX PerfTotalcount

MAX-ACCESS read-only

STATUS deprecated

DESCRIPTION

"The number of Degraded Minutes (DMs) encountered

by a DS1 interface in the previous 24-hour

interval. Invalid 15-minute intervals count as

$0 . "$

$::=\{$ dsxifarEndTotalEntry 10$\}$

-- The DS1 Fractional Table

dsx1Fractable OBJECT-TYPE

SYNTAX SEQUENCE OF DSXIFracEntry

MAX-ACCESS not-accessible

STATUS deprecated

DESCRIPTION

"This table is deprecated in favor of using

ifstackTable.

The table was mandatory for systems dividing a DS1

into channels containing different data streams

that are of local interest. Systems that are

indifferent to data content, such as CSUs, need

not implement it.

The DS1 Fractional table identifies which DS1

channels associated with a CSU are being used to

support a logical interface, i.e., an entry in the

interfaces table from the Internet-standard MIB.

For example, consider an application managing a North American ISDN Primary Rate link whose

division is a 384-kbit/s H1 _B_ Channel for video, 
a second $\mathrm{H} 1$ for data to a primary routing peer, and 12 64-kbit/s HO_B_Channels. Consider that some subset of the Ho channels is used for voice and the remainder are available for dynamic data calls.

We count a total of 14 interfaces multiplexed onto the DS1 interface. Six DS1 channels (for the sake of the example, channels 1..6) are used for video, six more (7..11 and 13) are used for data, and the remaining 12 are in channels 12 and 14..24.

Let us further imagine that ifIndex 2 is of type DS1 and refers to the DS1 interface and that the interfaces layered onto it are numbered 3..16.

We might describe the allocation of channels, in the dsxifractable, as follows:

dsxifracIfIndex. $2.1=3$ dsxifracIfIndex. $2.13=4$ dsxifracIfIndex.2. $2=3$ dsxifracIfIndex.2.14=6 dsxifracIfIndex.2. $3=3$ dsxifracIfIndex.2.15=7 dsxifracifIndex.2. $4=3$ dsxifracIfIndex.2.16=8 dsxifracIfIndex. $2.5=3$ dsxifracIfIndex.2.17=9 dsxifracIfIndex.2. $6=3$ dsxifracIfIndex.2.18=10 dsxifracIfIndex.2. $7=4$ dsxifracIfIndex.2.19= 11 dsxifracIfIndex.2. $8=4$ dsxifracIfIndex.2.20 $=12$ dsx1FracIfIndex.2. $9=4$ dsx1FracIfIndex.2.21 = 13 dsx1FracIfIndex.2.10=4 dsx1FracIfIndex.2.22=14 dsx1FracIfIndex.2.11 = 4 dsx1FracIfIndex.2.23= 15 dsx1FracIfIndex.2.12=5 dsx1FracIfIndex.2.24=16

For North American (DS1) interfaces, there are 24 legal channels, numbered 1 through 24 .

For G.704 interfaces, there are 31 legal channels, numbered 1 through 31. The channels (1..31) correspond directly to the equivalently numbered time-slots."

$$
::=\{d s 113\}
$$

dsX1FracEntry OBJECT-TYPE

SYNTAX DsX1FracEntry

MAX-ACCESS not-accessible

STATUS deprecated

DESCRIPTION

"An entry in the DS1 Fractional table."

INDEX \{ dsxifracIndex, dsxifracNumber

$::=\{$ dsxifractable 1$\}$ 


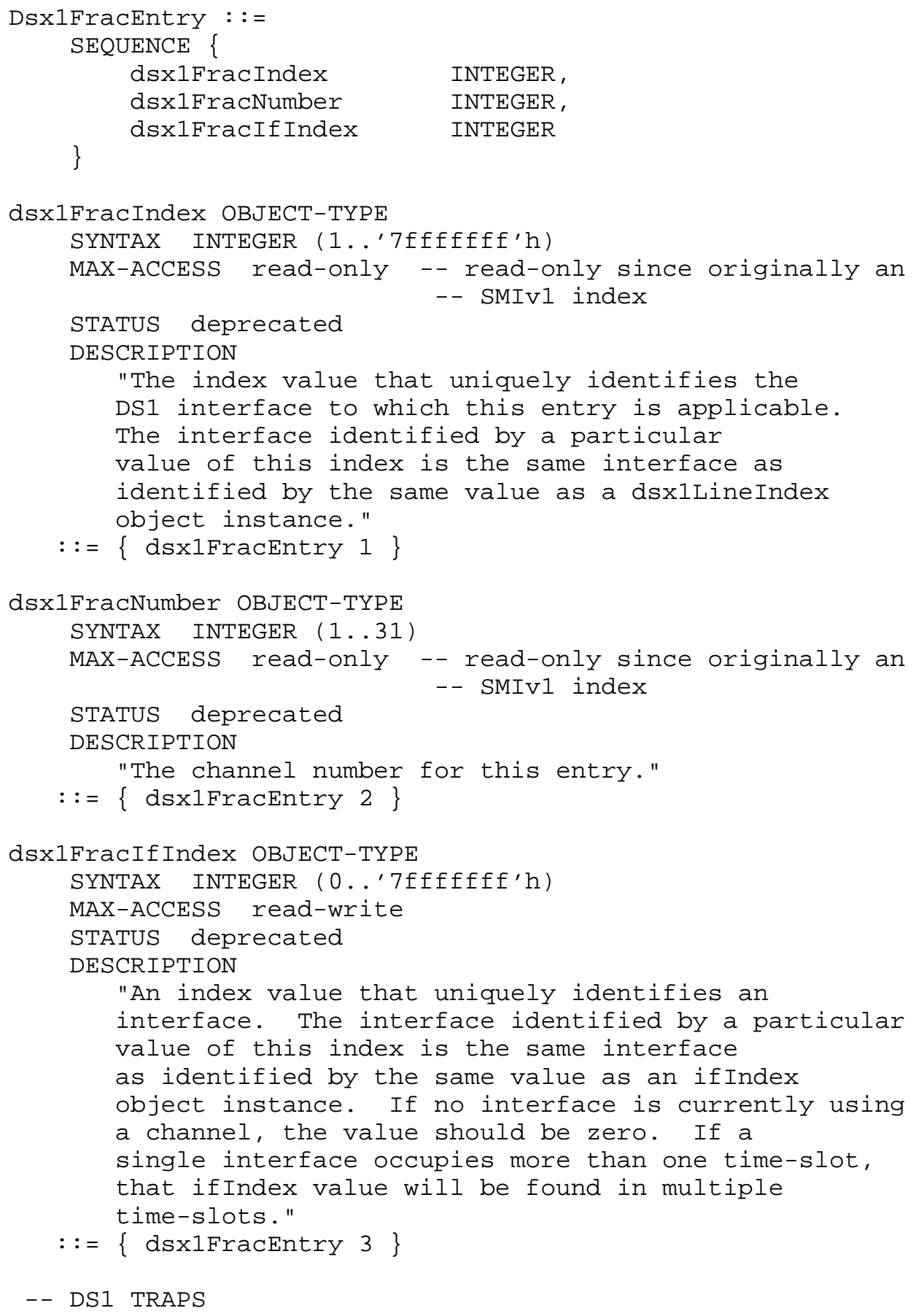




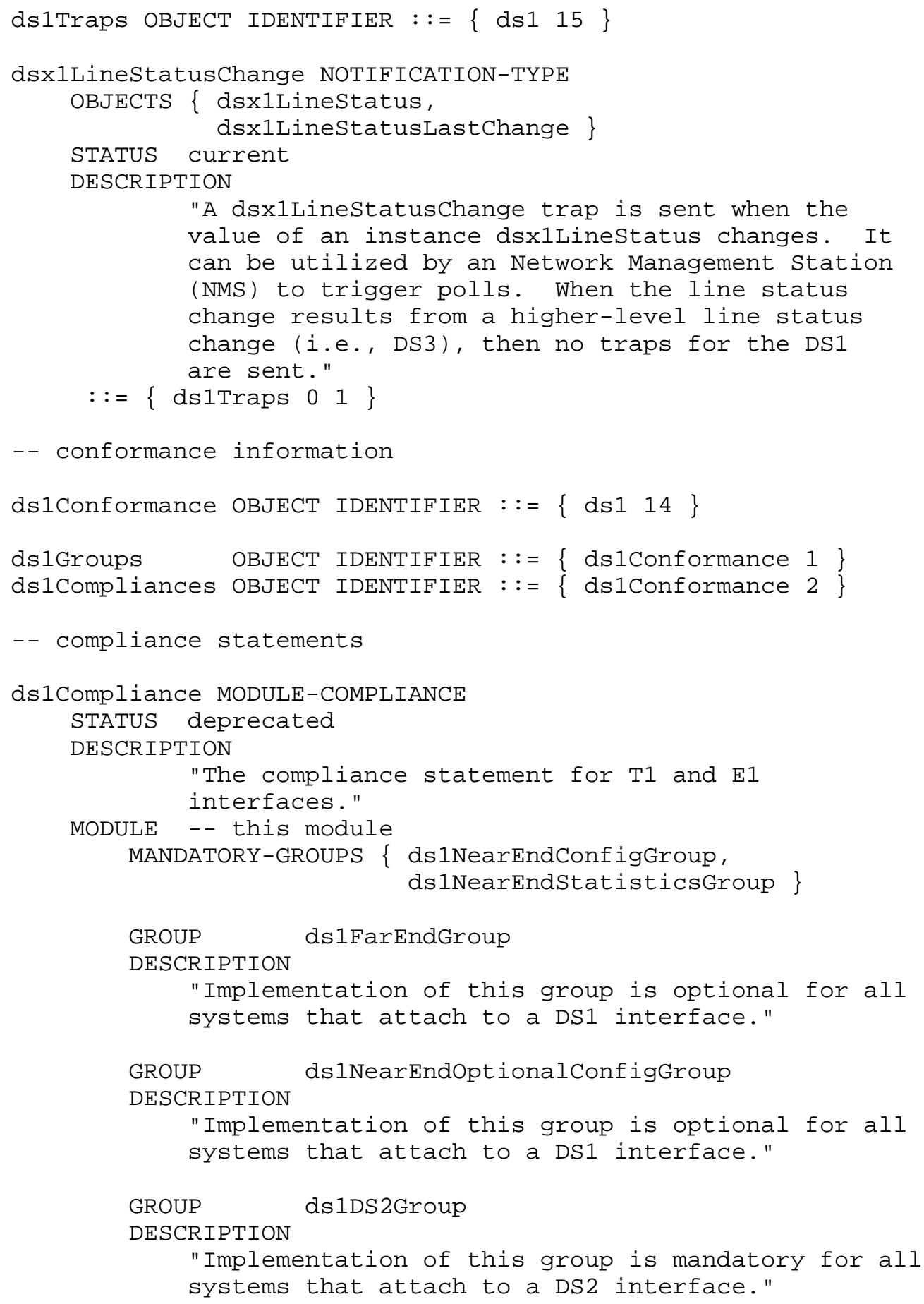




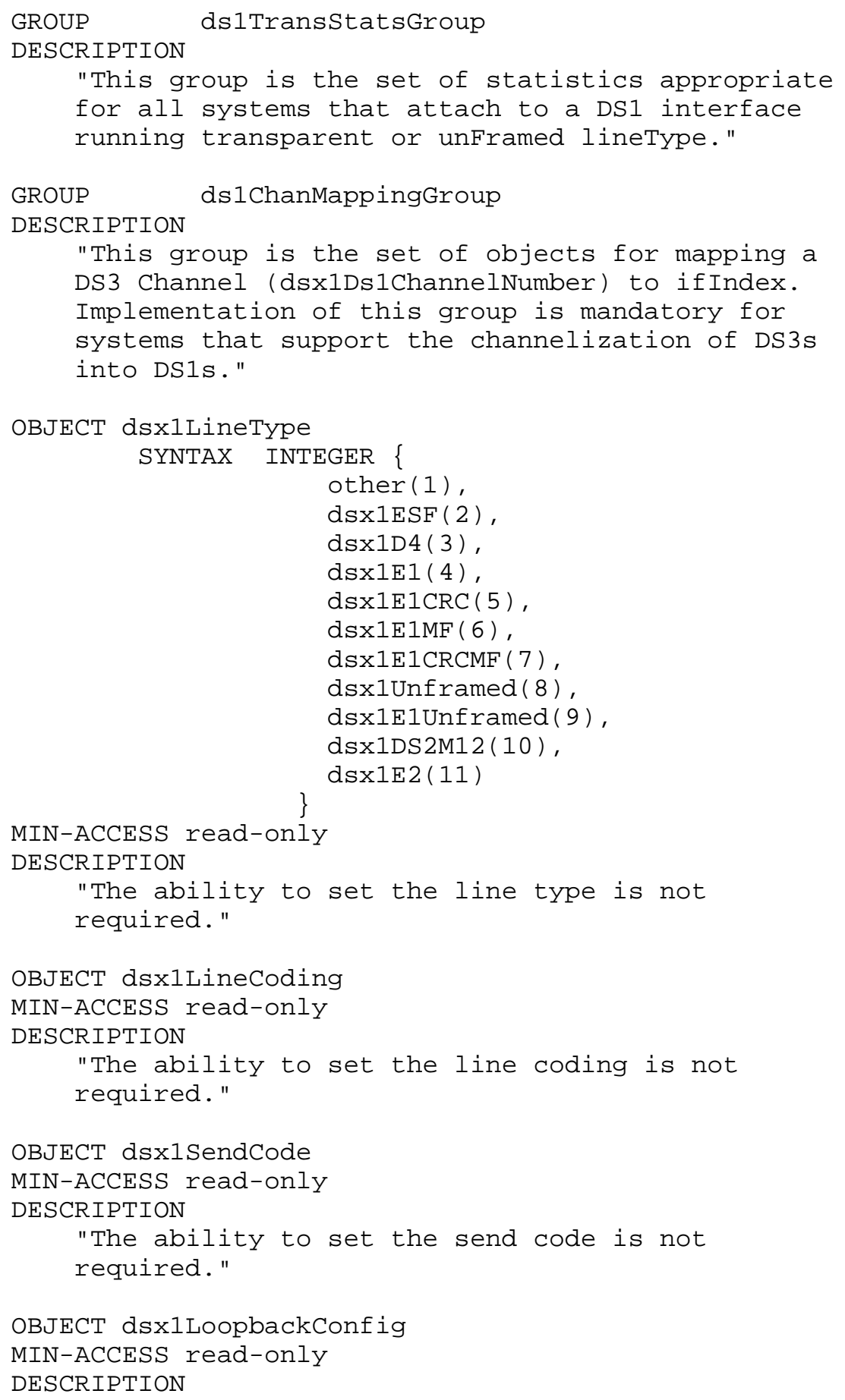


"The ability to set loopbacks is not required."

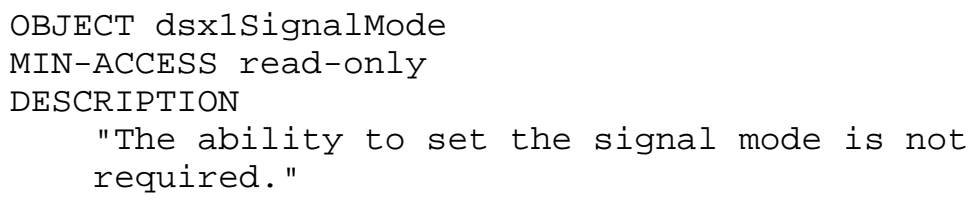




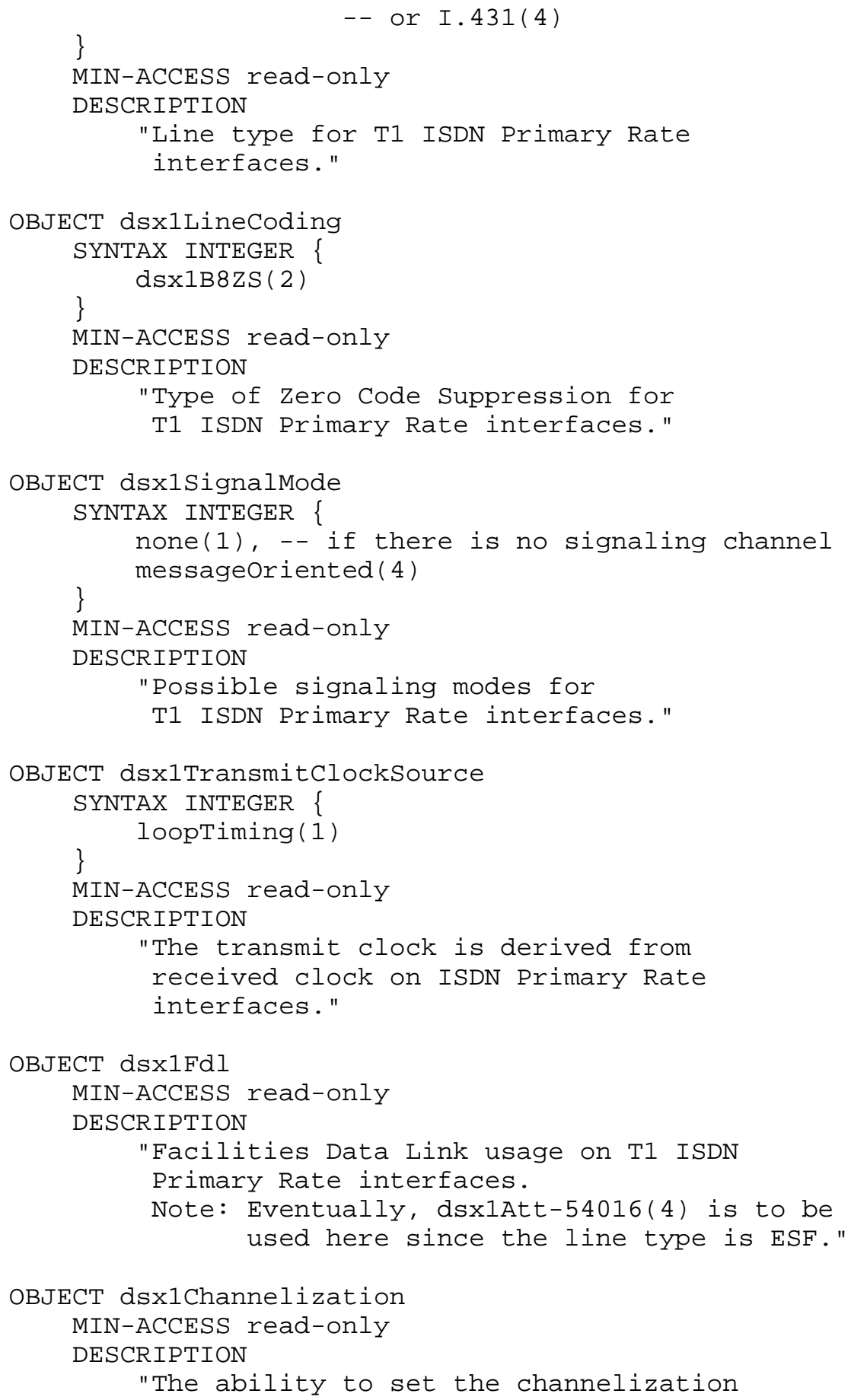




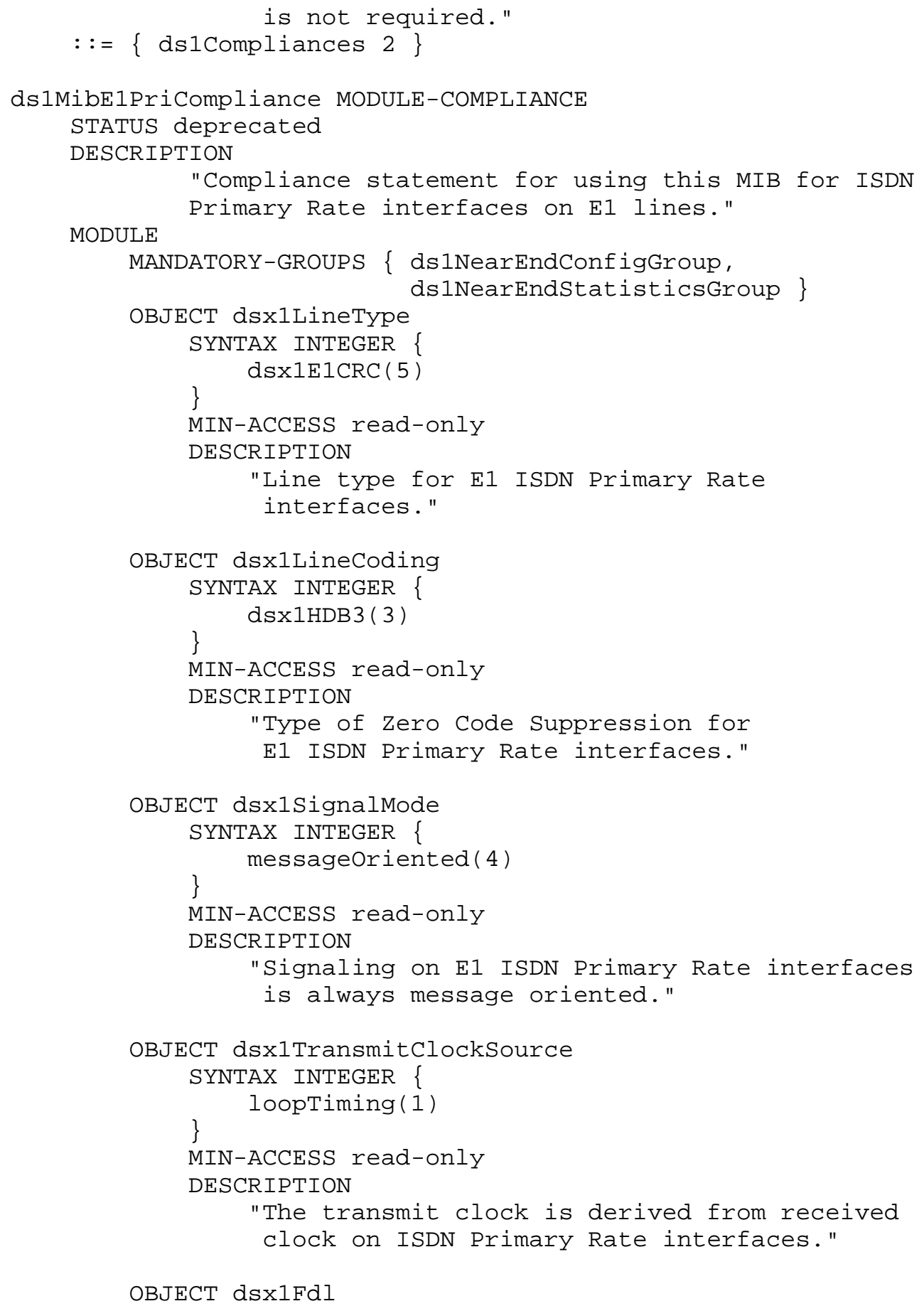




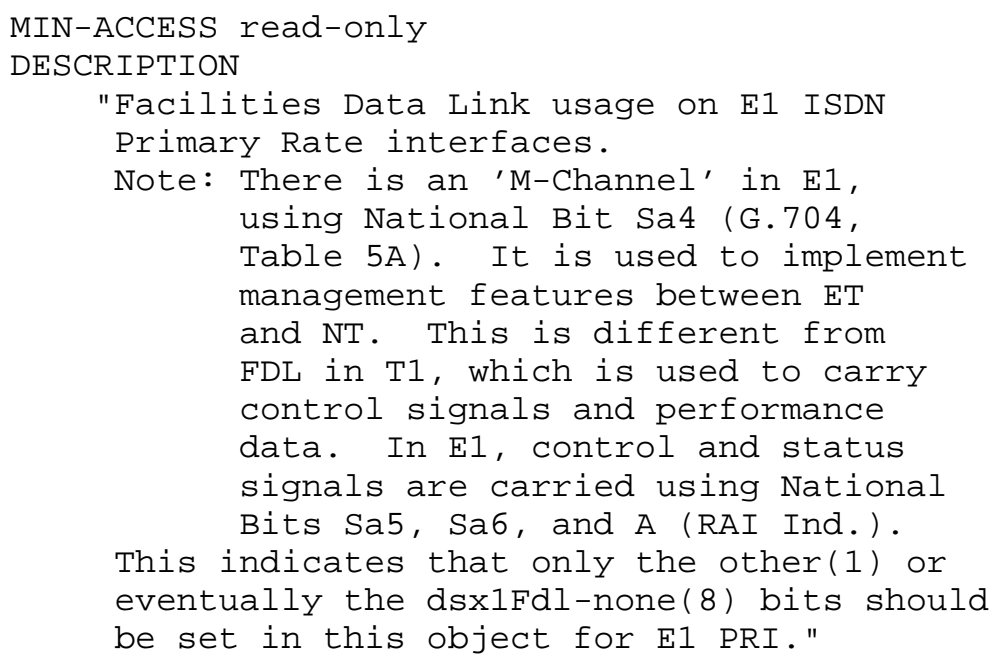


$::=\{$ ds1Compliances 4$\}$

ds1NCompliance MODULE-COMPLIANCE

STATUS deprecated

DESCRIPTION

"The compliance statement for $\mathrm{T} 1$ and $\mathrm{E} 1$

interfaces."

MODULE -- this module

MANDATORY-GROUPS \{ ds1NearEndConfigurationGroup, ds1NearEndStatisticsGroup \}

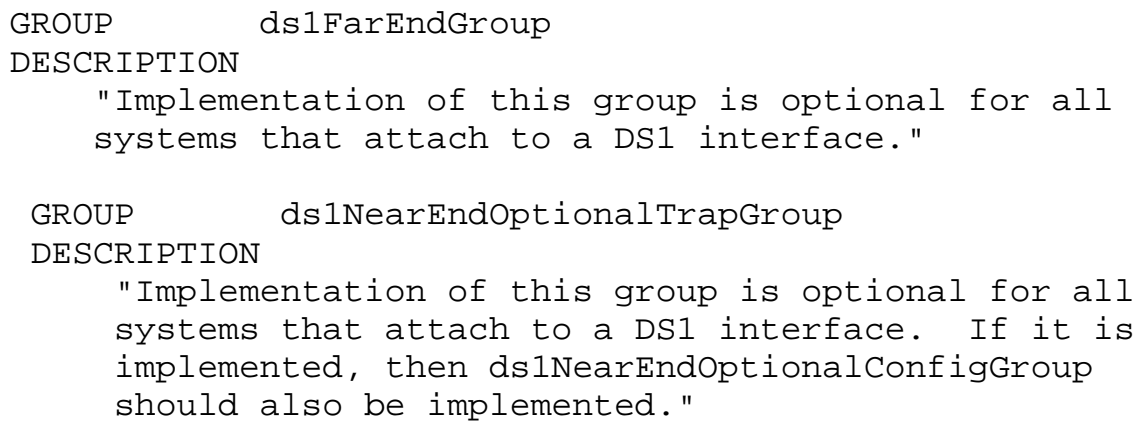




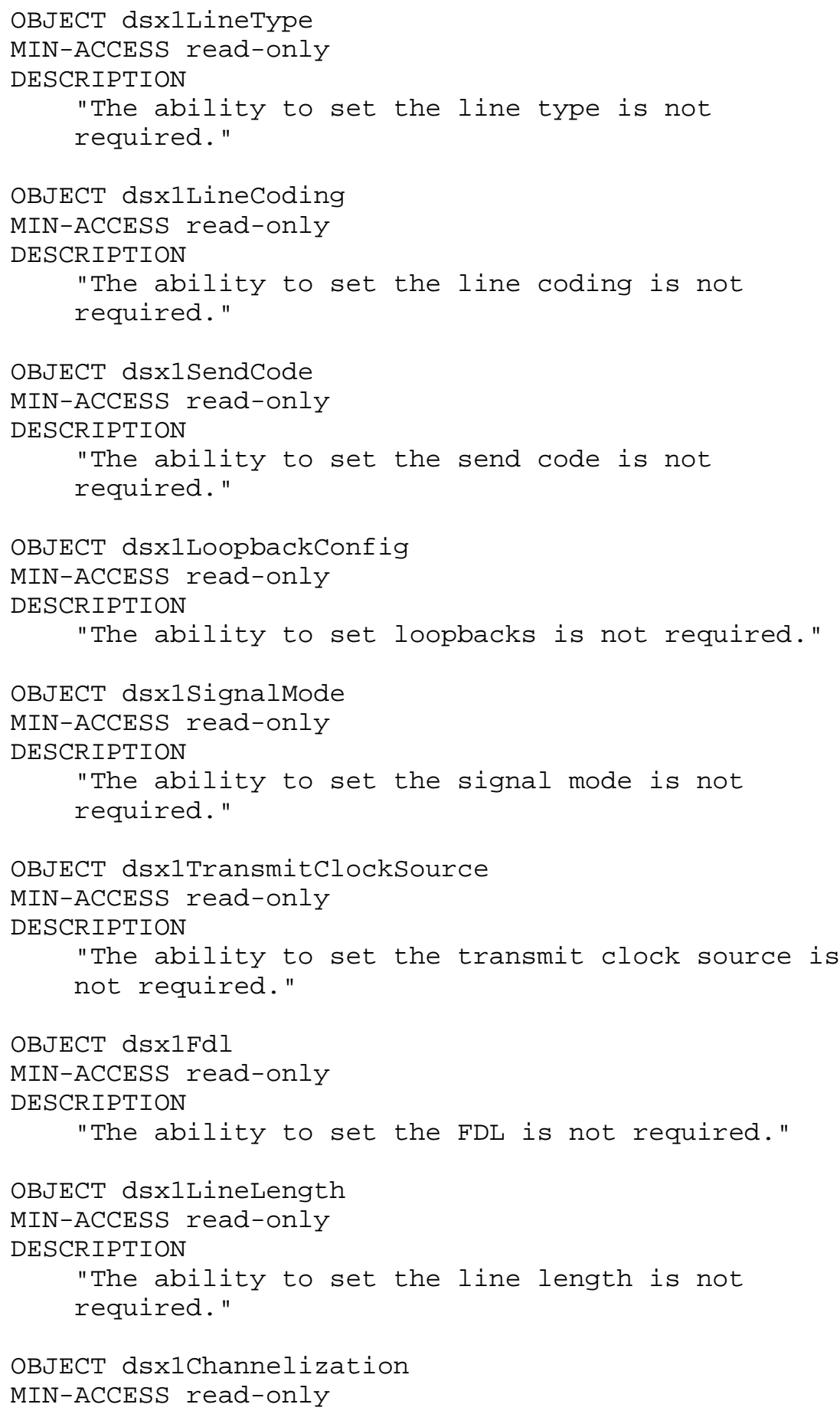




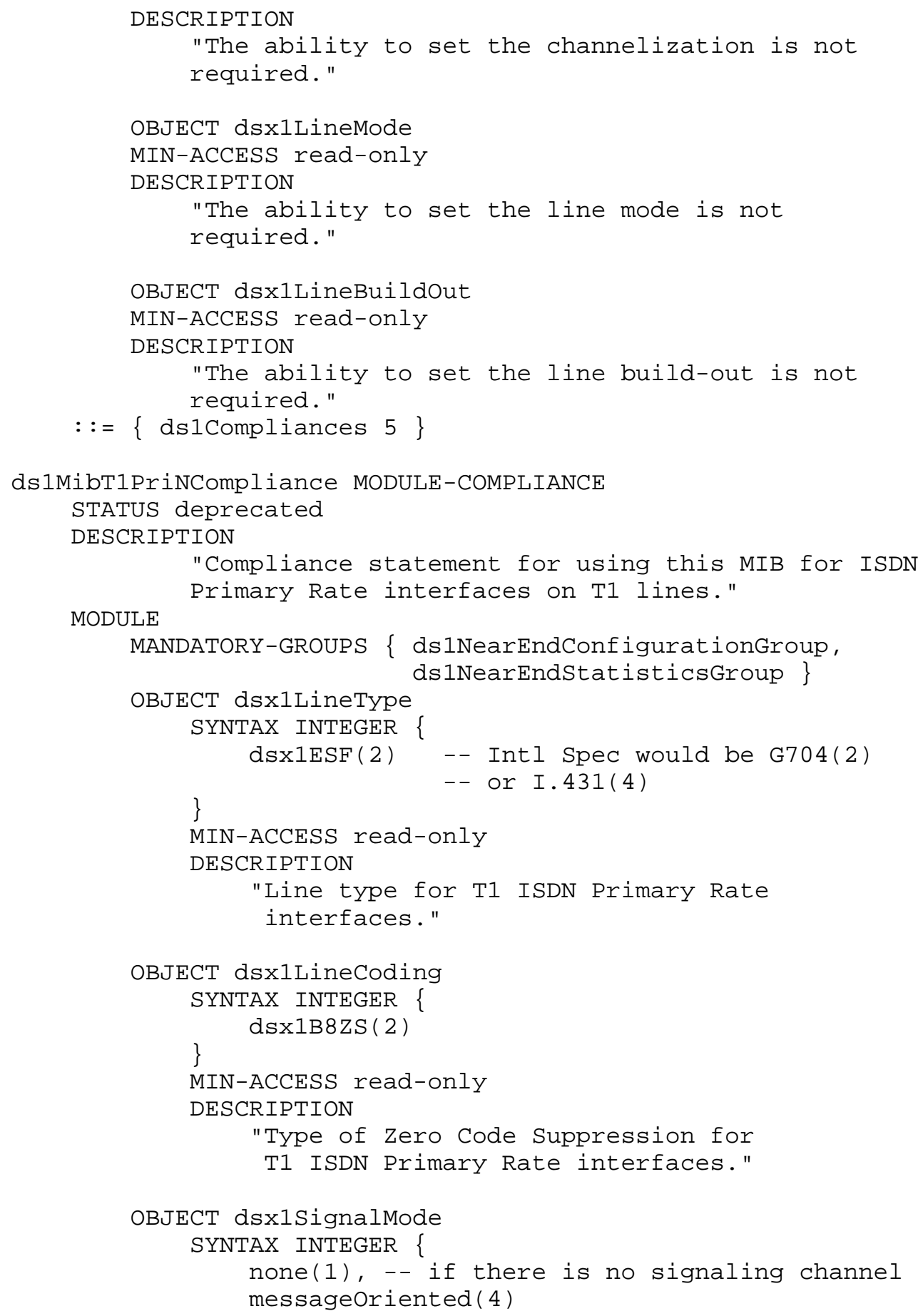




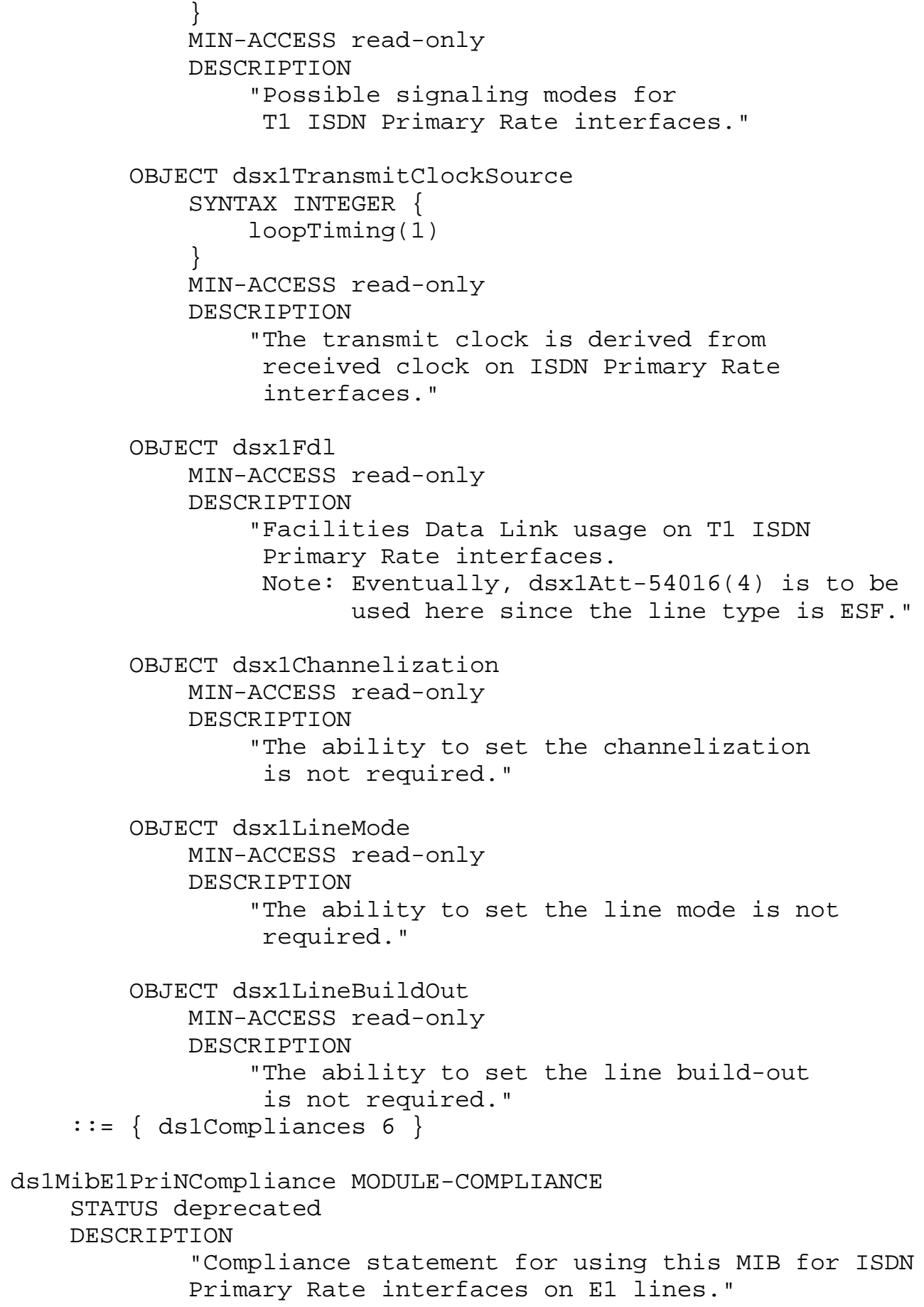




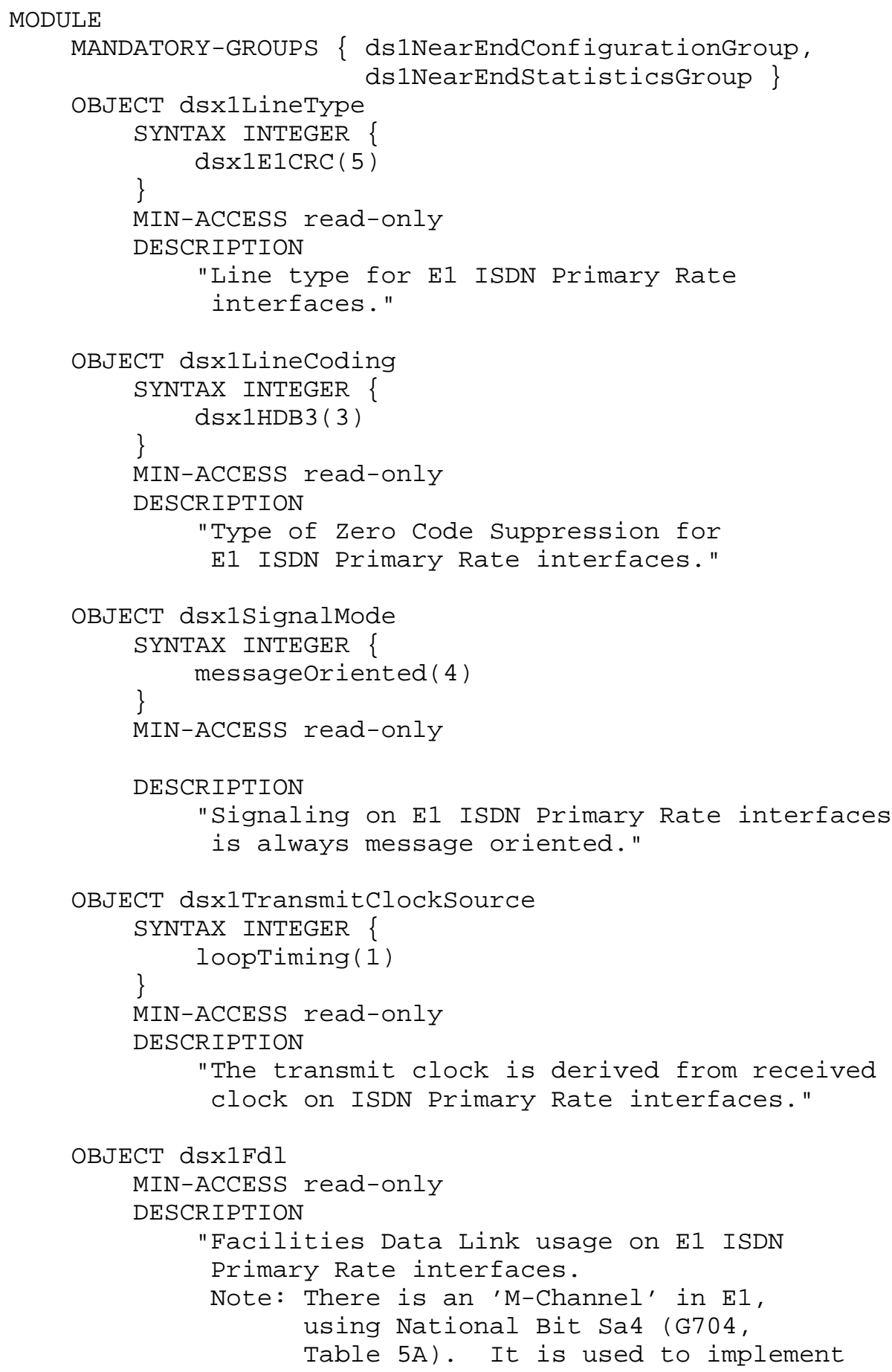




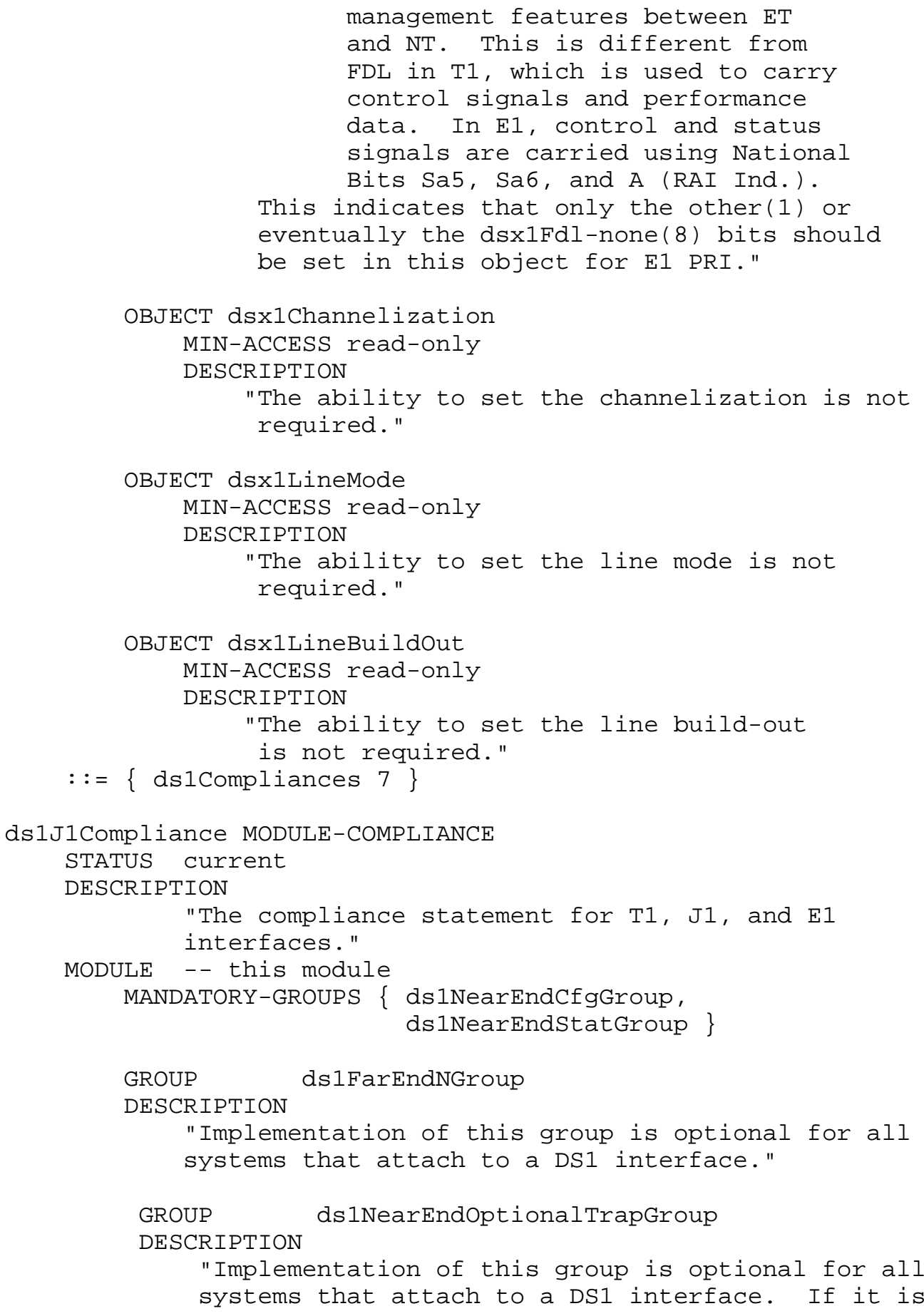




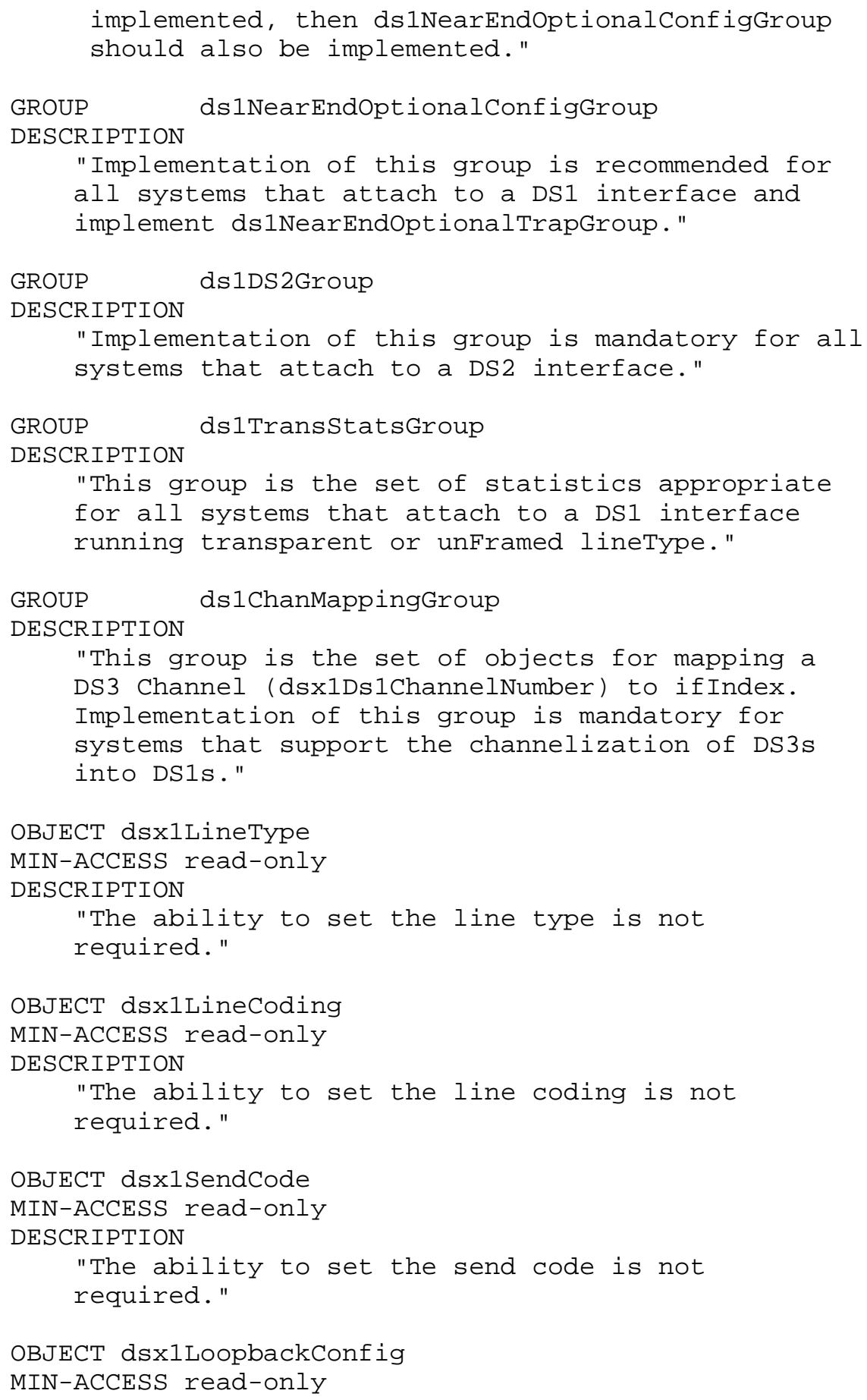




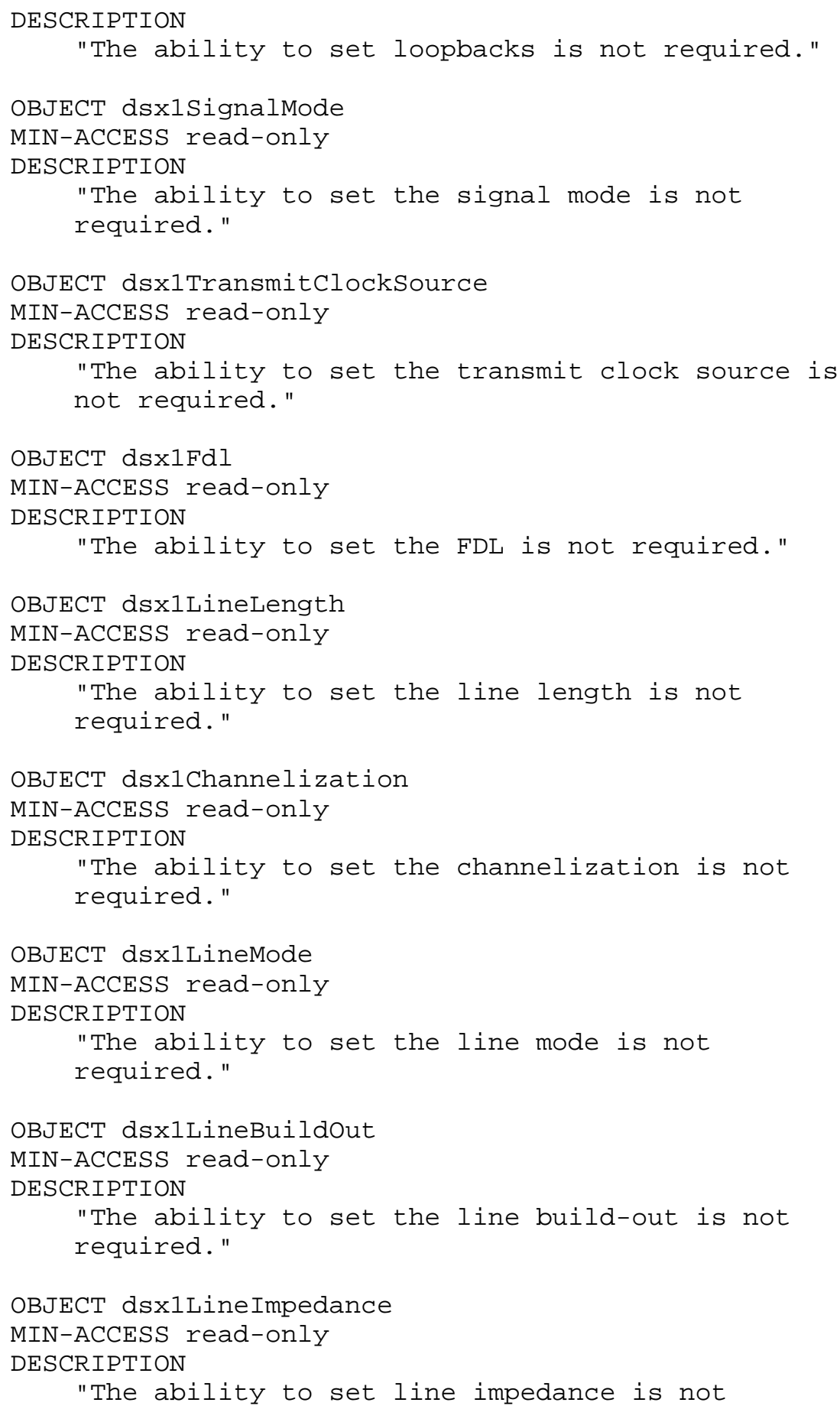




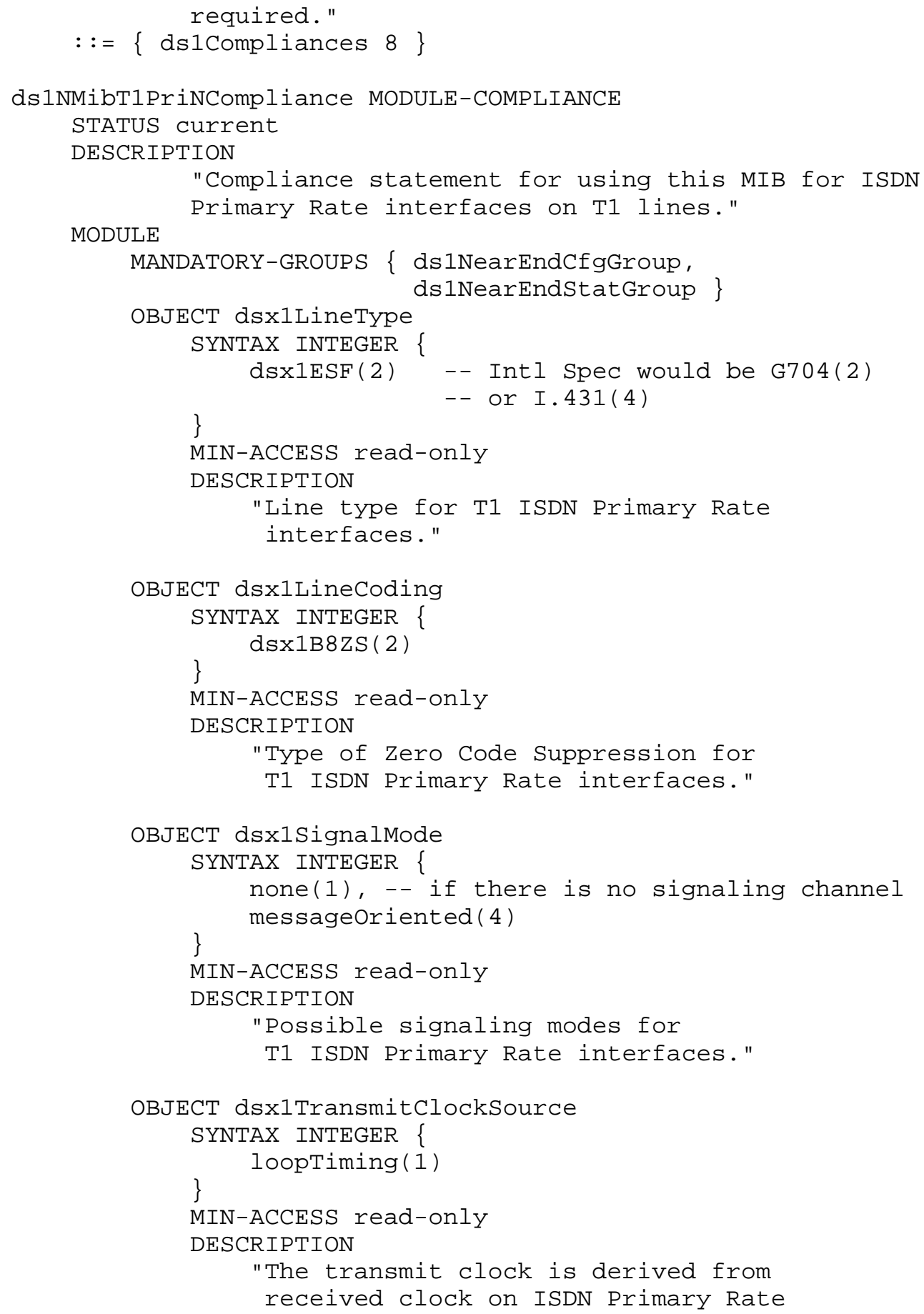




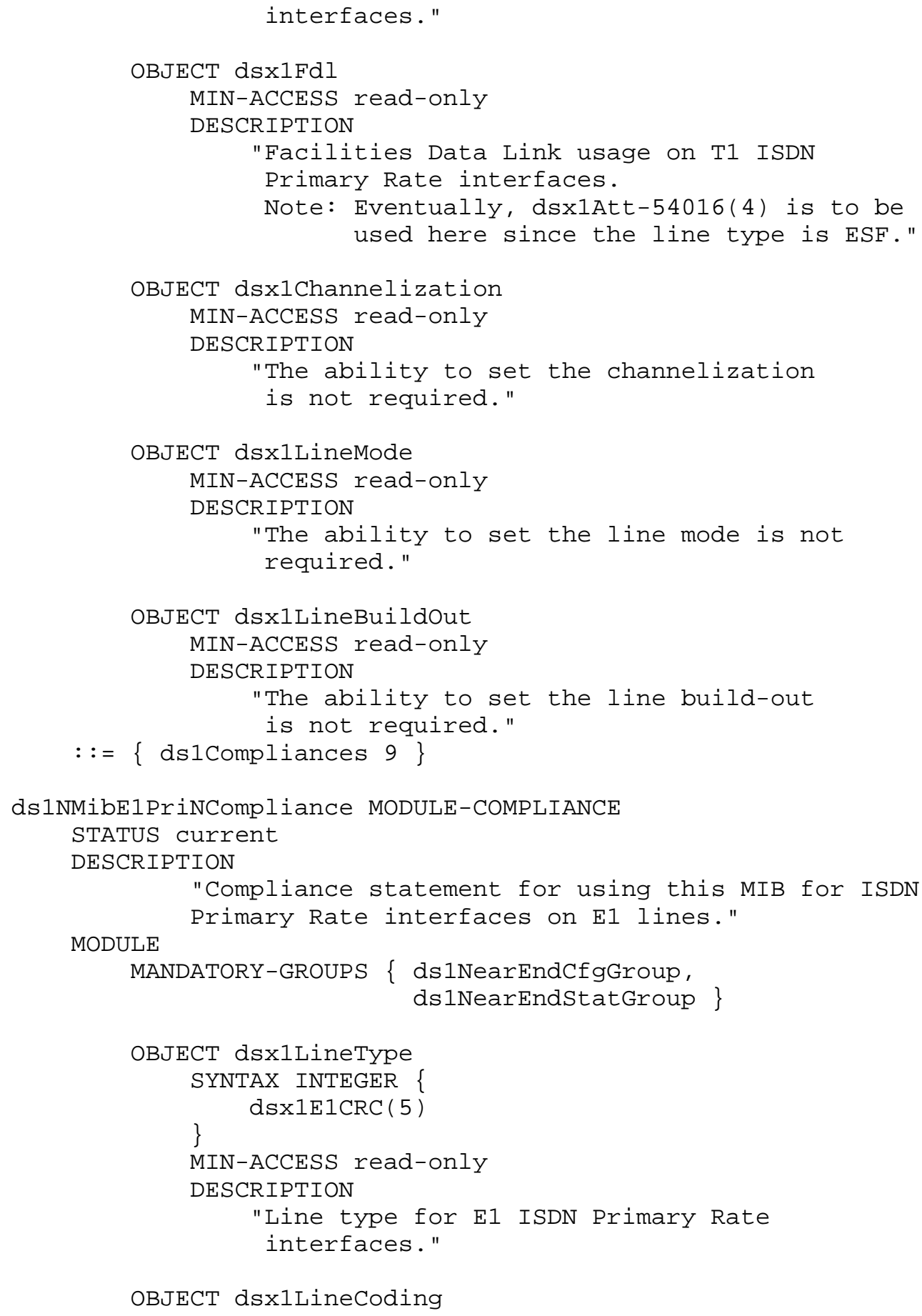




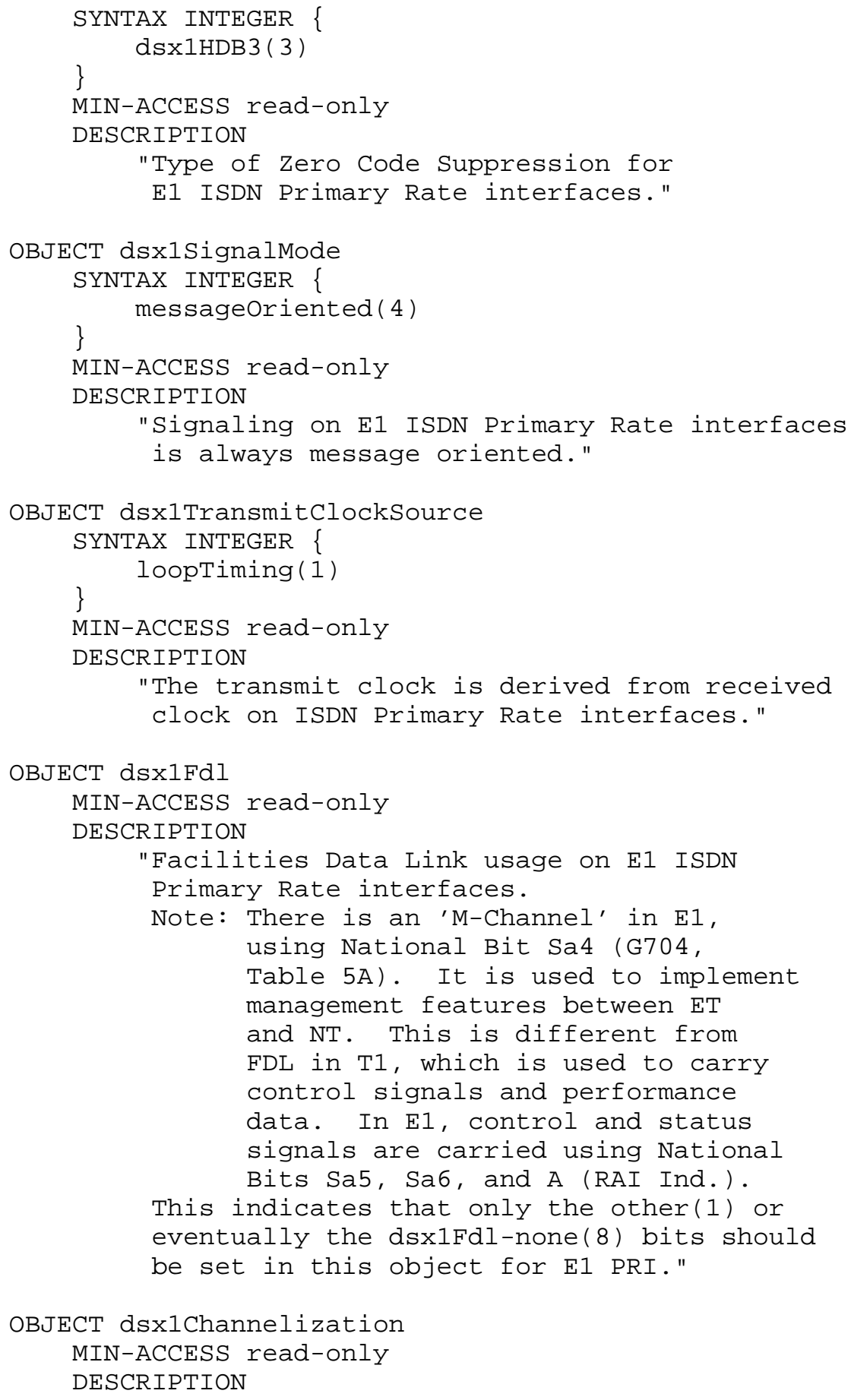




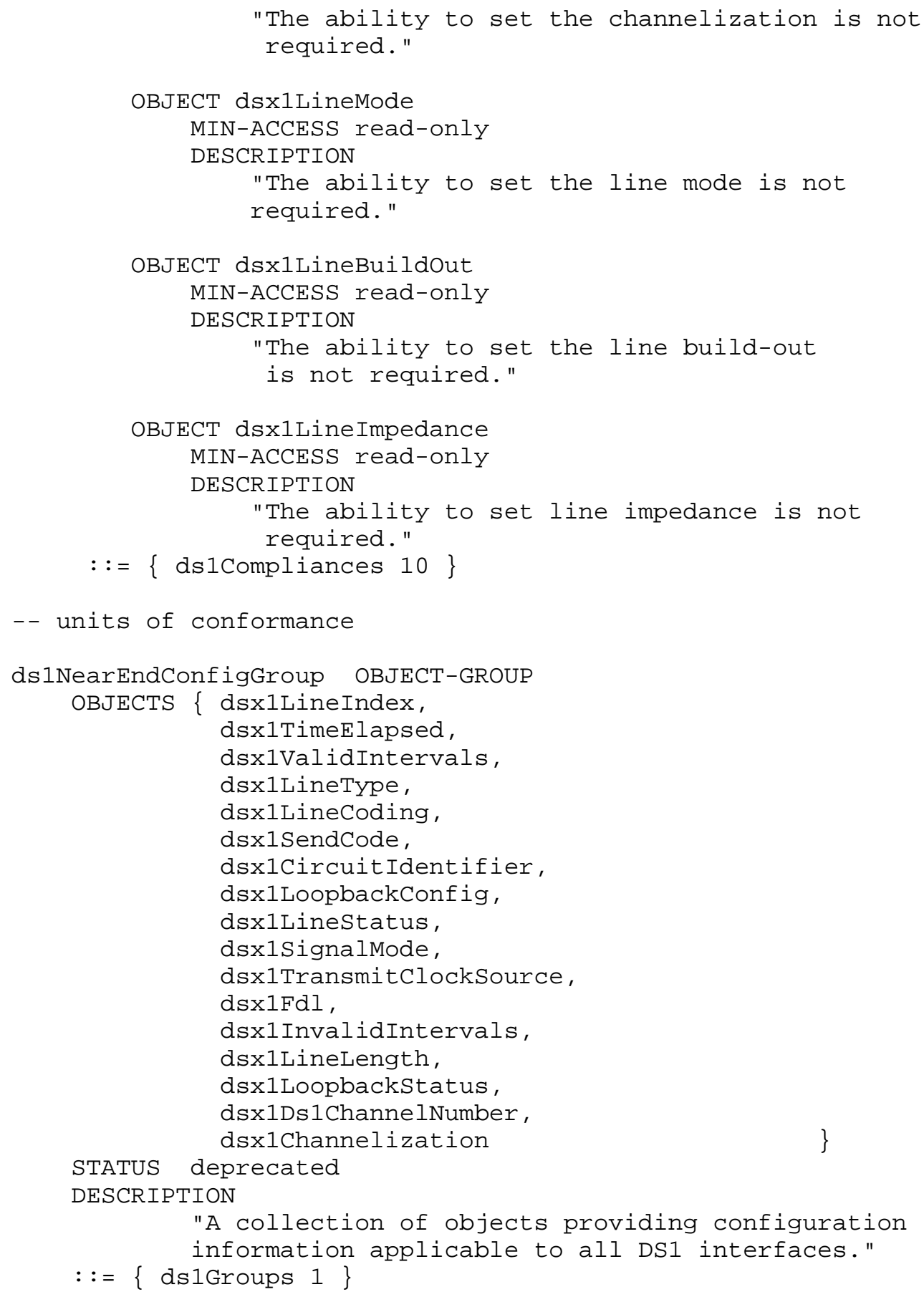




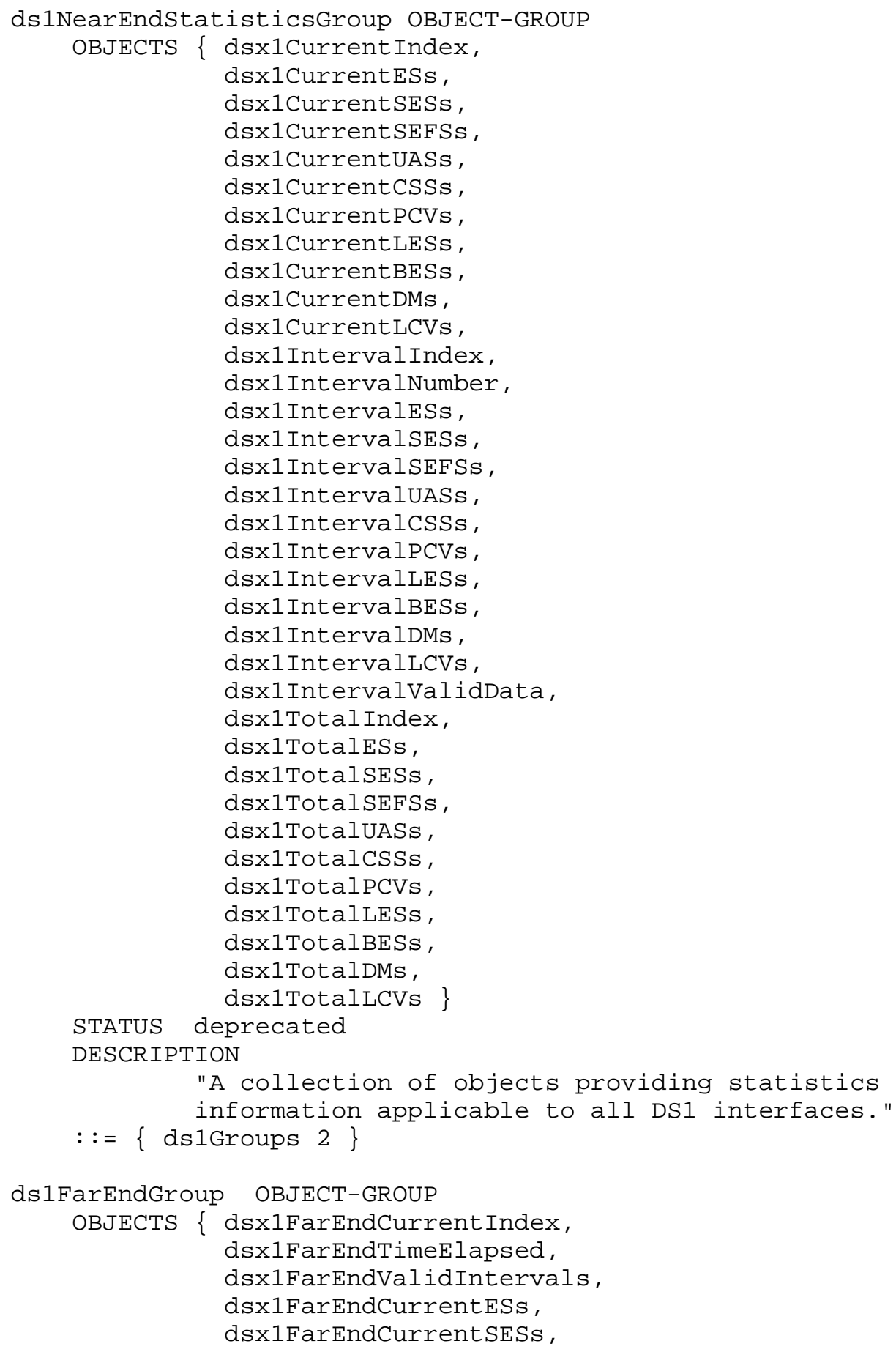




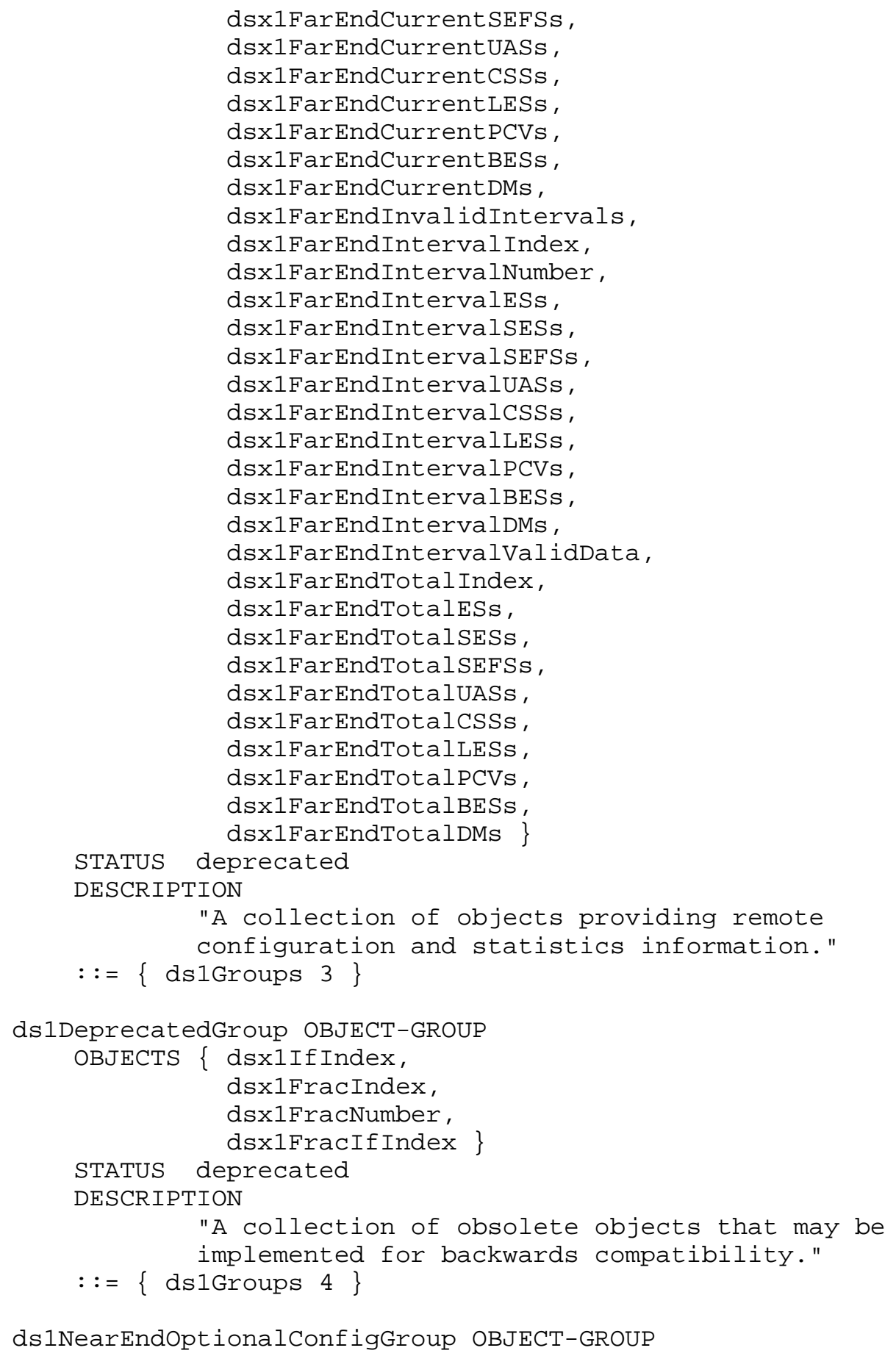




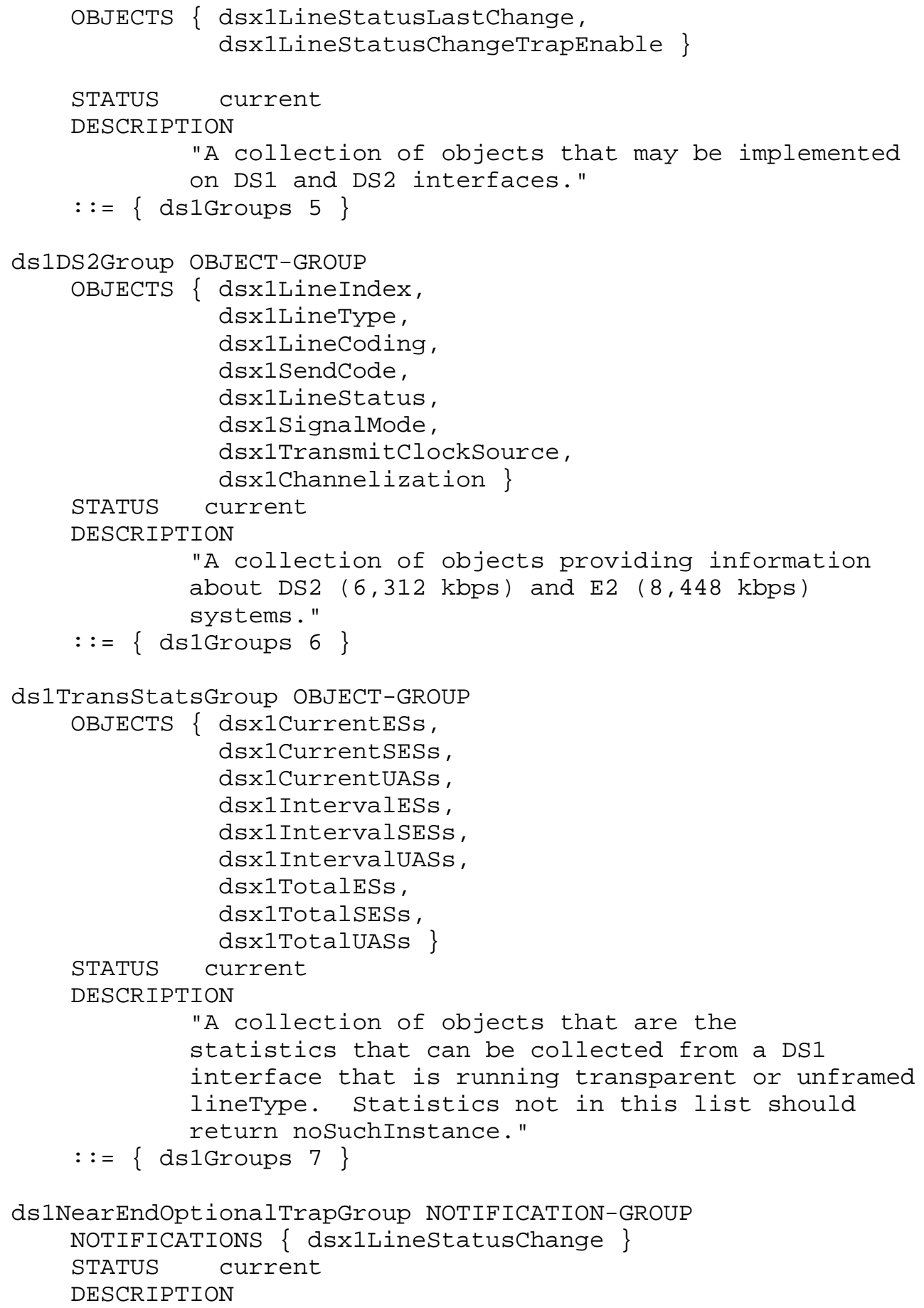




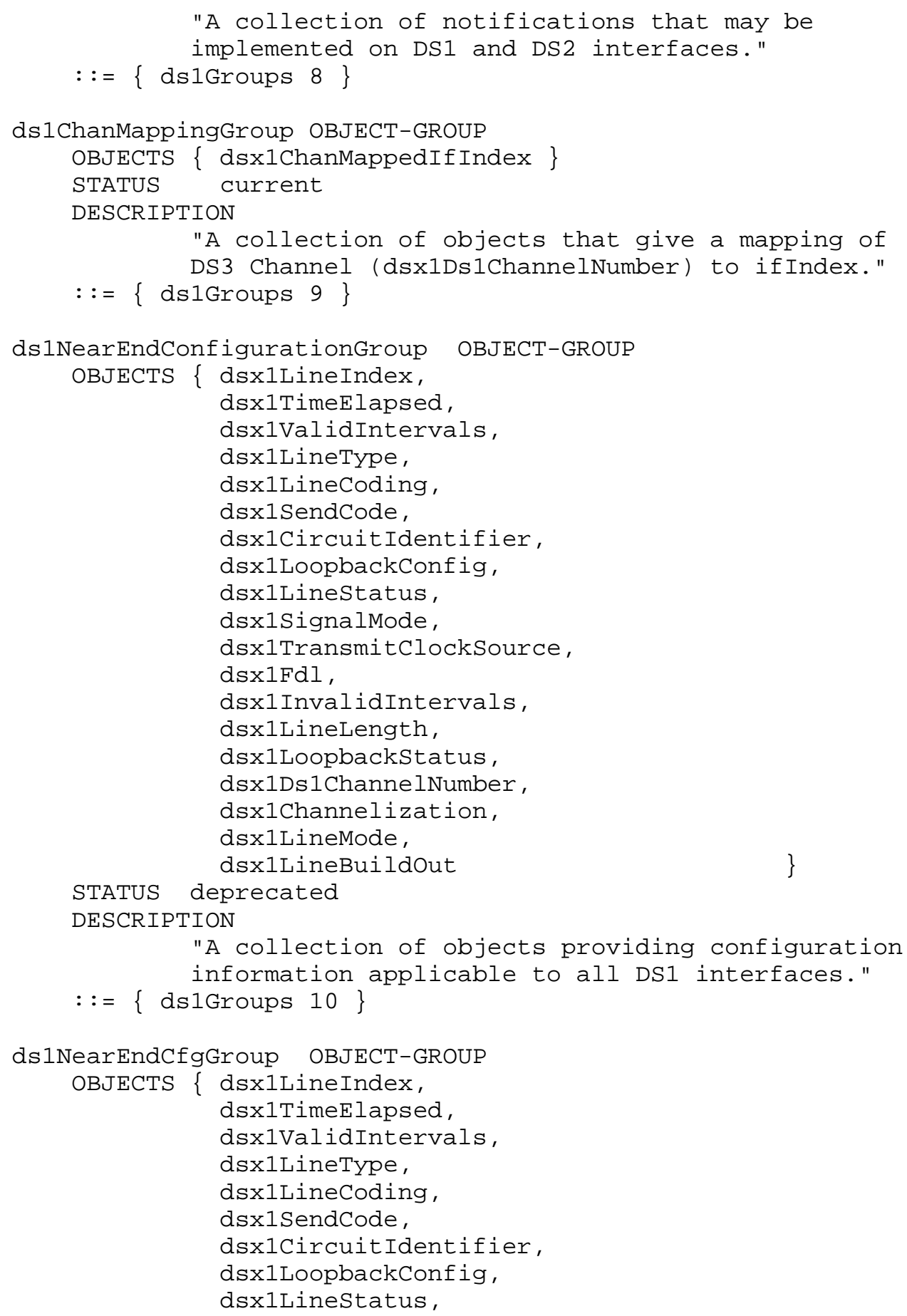




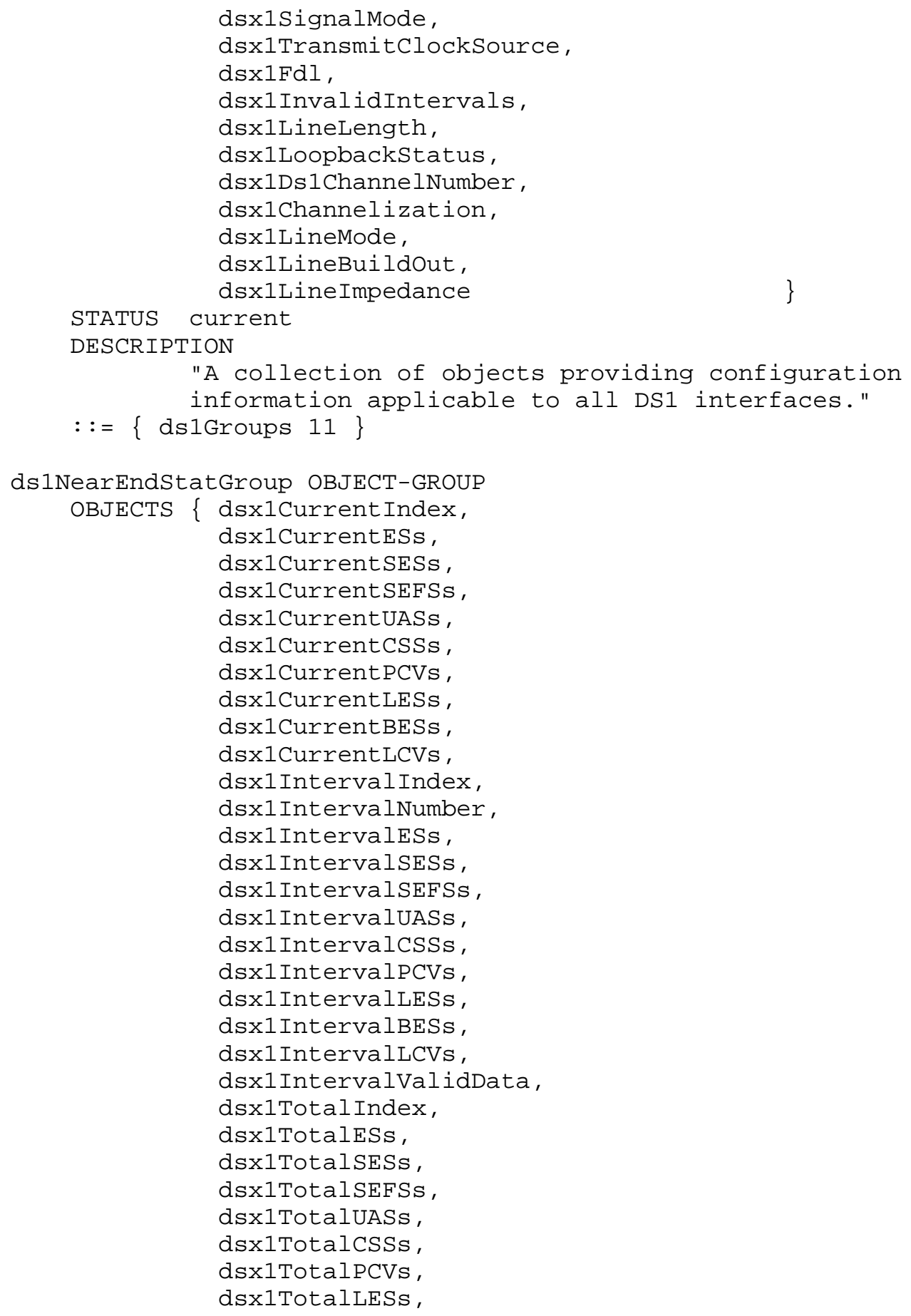




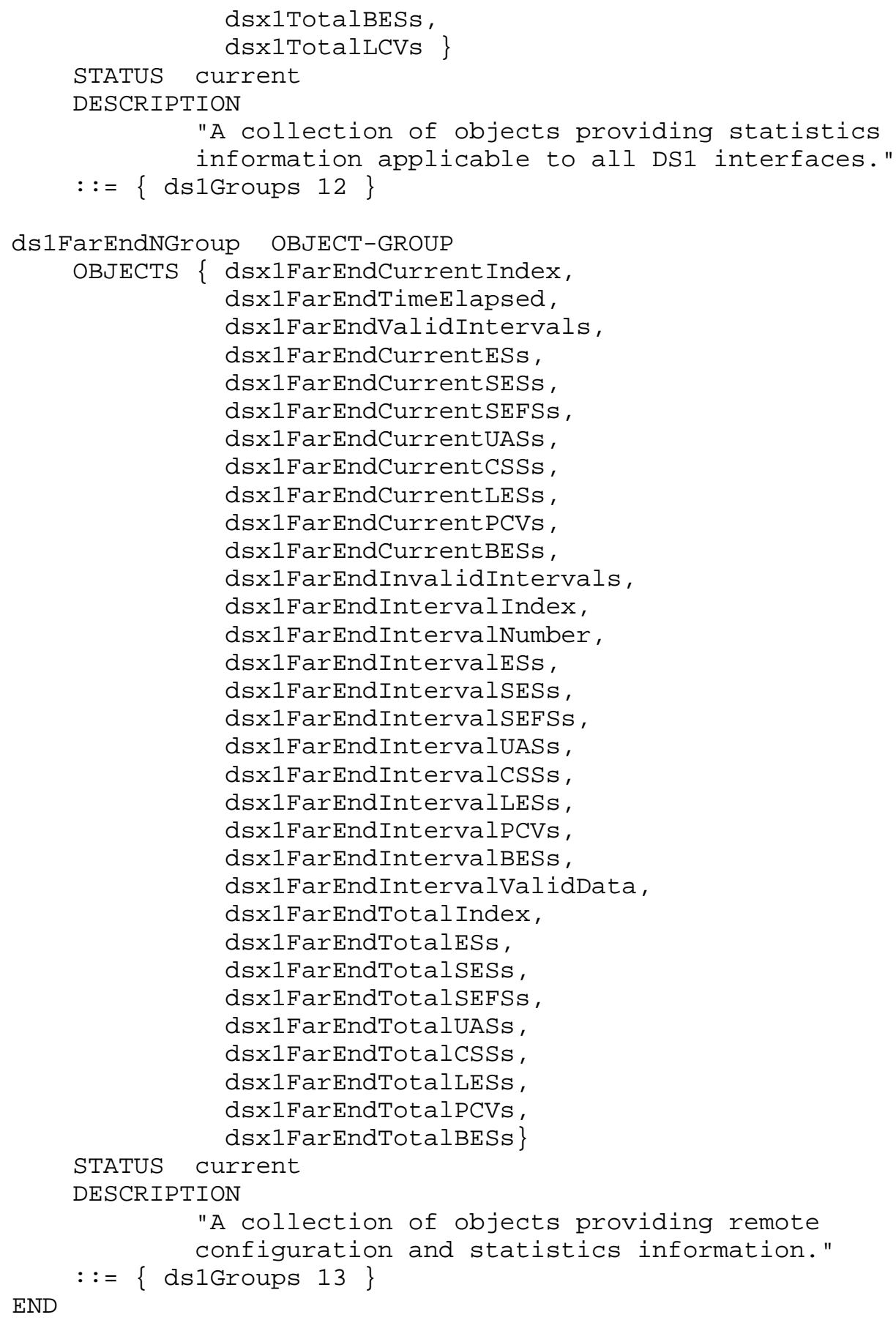




\section{Security Considerations}

There are a number of management objects defined in this MIB module with a MAX-ACCESS clause of read-write. Such objects may be considered sensitive or vulnerable in some network environments. The support for SET operations in a non-secure environment without proper protection can have a negative effect on network operations. The specific objects and their sensitivities/vulnerabilities are as follows.

Setting the following objects to incorrect values may result in traffic interruptions:

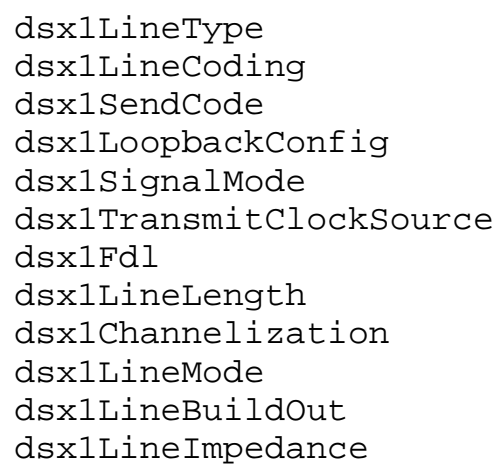

In the case of dsxlLineType, for example, both ends of a DS1/E1 must have the same value in order for traffic to flow. In the case of dsxisendCode and dsxlLoopbackConfig, for another example, traffic may stop transmitting when particular loopbacks are applied.

Setting the following object to an incorrect value will not harm the traffic, but it may cause a circuit to be misidentified and thereby create difficulties for service personnel when attempting to troubleshoot a problem:

dsx1CircuitIdentifier

Setting the following object can cause an increase in the number of traps received by the network management station:

dsxilineStatusChangeTrapEnable

The readable objects in this MIB module (i.e., the objects with a MAX-ACCESS other than not-accessible) may be considered sensitive in some environments since, collectively, they provide extensive information about the performance of interfaces in DS1/J1/E1/DS2/E2 equipment or networks and can reveal some aspects of their 
configuration. In such environments, it is important to control even GET and NOTIFY access to these objects and possibly to encrypt the values of these objects when sending them over the network via SNMP.

SNMP versions prior to SNMPV3 did not include adequate security. Even if the network itself is secure (for example by using IPSec), even then, there is no control as to who on the secure network is allowed to access and GET/SET (read/change/create/delete) the objects in this MIB module.

It is RECOMMENDED that implementers consider the security features as provided by the SNMPV3 framework (see [RFC3410], section 8), including full support for the SNMPv3 cryptographic mechanisms (for authentication and privacy).

Further, deployment of SNMP versions prior to SNMPv3 is NOT RECOMMENDED. Instead, it is RECOMMENDED to deploy SNMPv3 and to enable cryptographic security. It is then a customer/operator responsibility to ensure that the SNMP entity giving access to an instance of this MIB module is properly configured to give access to the objects only to those principals (users) that have legitimate rights to indeed GET or SET (change/create/delete) them.

6. Acknowledgments

This document was produced by the ATOM MIB Working Group.

7. References

7.1. Normative References

[RFC2119] Bradner, S., "Key words for use in RFCs to Indicate Requirement Levels", BCP 14, RFC 2119, March 1997.

[RFC2578] MCCloghrie, K., Perkins, D., and J. Schoenwaelder, "Structure of Management Information Version 2 (SMIV2)", STD 58, RFC 2578, April 1999.

[RFC2579] McCloghrie, K., Perkins, D., and J. Schoenwaelder, "Textual Conventions for SMIv2", STD 58, RFC 2579, April 1999.

[RFC2580] McCloghrie, K., Perkins, D., and J. Schoenwaelder, "Conformance statements for SMIv2", STD 58, RFC 2580, April 1999.

[RFC2863] McCloghrie, K. and F. Kastenholz, "The Interfaces Group MIB", RFC 2863, June 2000 . 
[AT\&T-TR-54016] AT\&T Technical Reference, Requirements for Interfacing Digital Terminal Equipment to Services Employing the Extended Superframe Format, Publication 54016, May 1988 .

[ANSI-T1.403] American National Standard for Telecommunications -Carrier-to-Customer Installation - DS1 Metallic Interface, T1.403, February 1989.

[CCITT-G.703] ITU-T G.703, Physical/Electrical Characteristics of Hierarchical Digital Interfaces, November 2001.

[ITU-T-G.704] ITU-T G.704: Synchronous frame structures used at 1544, 6312, 2048, 8488 and 44736 kbit/s Hierarchical Levels, October 1998 .

[ANSI-T1.231] American National Standard for Telecommunications -Digital Hierarchy DS1-- Layer 1 In-Service Digital Transmission Performance Monitoring, T1.231.02, october 2003 .

[ITU-T-0.162] ITU-T 0.162, Equipment To Perform In Service Monitoring On $2048 \mathrm{kbit} / \mathrm{s}$ Signals, October 1992.

[CCITT-G.821] ITU-T G.821, Error Performance of An International Digital Connection Forming Part Of An Integrated Services Digital Network, December 2002.

[AT\&T-TR-62411] AT\&T Technical Reference, Technical Reference 62411, ACCUNET T1.5 Service Description And Interface Specification, December 1990.

[CCITT-G.706] ITU-T G.706, Frame Alignment and Cyclic Redundancy Check (CRC) Procedures Relating to Basic Frame Structures Defined in Recommendation G.704, April 1991 .

[CCITT-G.732] ITU-T G.732, Characteristics of Primary PCM Multiplex Equipment Operating at $2048 \mathrm{kbit} / \mathrm{s}$, November 1988 .

[ITU-T-G.775] ITU-T G.775: Loss of signal (LOS) and alarm indication signal (AIS) defect detection and clearance criteria, October 1998.

[ITU-T-G.826] ITU-T G.826: Error performance parameters and objectives for international, constant bit rate digital paths at or above the primary rate, December 2002 . 
[ANSI-T1.107]

American National Standard for Telecommunications -Digital Hierarchy - Format Specifications, T1.107, January 2002 .

[RFC3593]

Tesink, K., "Textual Conventions for MIB Modules Using Performance History Based on 15 Minute Intervals", RFC 3593, September 2003.

[ITU-T-M.1400] ITU-T M.1400: Designation For Interconnections Among Network Operators, October 2001.

$[\mathrm{JT}-\mathrm{G} 704]$ JT-G.704: Synchronous frame structures used at Primary and Secondary Hierarchical Levels, 2002.

[JT-G706] JT-G.706: Frame Alignment and Cyclic Redundancy Check (CRC) Procedures.

[JT-I431] JT-I.431: ISDN Primary Rate User-Network Interface, Layer 1 Specifications, 2002.

7.2. Informative References

[RFC1213]

McCloghrie, K. and M. Rose, "Management Information Base for Network Management of TCP/IP-based internets:MIB-II", STD 17, RFC 1213, March 1991.

[RFC3895]

Nicklass, 0., "Definitions of Managed Objects for the DS1, E1, DS2, and E2 Interface Types", RFC 3895, September 2004 .

[RFC2495] Fowler, D., "Definitions of Managed Objects for the DS1, E1, DS2 and E2 Interface Types", RFC 2495, January 1999 .

[RFC1406] Baker, F. and J. Watt, "Definitions of Managed Objects for the DS1 and E1 Interface Types", RFC 1406, January 1993.

[AT\&T-UM-305] AT\&T Information Systems, AT\&T ESF DS1 Channel Service Unit User's Manual, 999-100-305, February 1988.

[RFC3896] Nicklass, O., "Definitions of Managed Objects for the DS3/E3 Interface Type", RFC 3896, September 2004. 
[RFC3592]

[RFC2494]

Tesink, K., "Definitions of Managed objects for the Synchronous Optical Network/Synchronous Digital Hierarchy (SONET/SDH) Interface Type", RFC 3592, September 2003.

[ANSI-T1.102]

[RFC3410]
Fowler, D., "Definitions of Managed objects for the DSO and DSO Bundle Interface Type", RFC 2494, January 1999 .

American National Standard for Telecommunications -Digital Hierarchy - Electrical Interfaces, T1.102, December 1993.

Case, J., Mundy, R., Partain, D., and B. Stewart, "Introduction and Applicability Statements for

Internet-Standard Management Framework", RFC 3410, December 2002 . 
Appendix A - Use of dsxlIfIndex and dsxlLineIndex

This appendix exists to document the previous use of dsxlIfIndex and dsxlLineIndex and to clarify the relationship of dsxlLineIndex as defined in RFC 1406 with the dsxlLineIndex as defined in this document.

The following shows the old and new definitions and the relationship:

[New Definition]: "This object should be made equal to ifIndex. The next paragraph describes its previous usage. Making the object equal to ifIndex allows proper use of ifstackTable and ds0/ds0bundle mibs.

[Old Definition]: "This object is the identifier of a DS1 Interface on a managed device. If there is an ifEntry that is directly associated with this and only this DS1 interface, it should have the same value as ifIndex. Otherwise, number the dsxlLineIndices with an unique identifier following the rules of choosing a number that is greater than ifNumber and numbering the inside interfaces (e.g., equipment side) with even numbers and outside interfaces (e.g., network side) with odd numbers."

When the "Old Definition" was created, it was described this way to allow a manager to treat the value as if it were an ifIndex; i.e., the value would be either: 1) an ifIndex value or 2) a value that was guaranteed to be different from all valid ifIndex values.

The new definition is a subset of that definition; i.e., the value is always an ifIndex value.

The following is section 3.1 from RFC 1406:

Different physical configurations for the support of SNMP with DS1 equipment exist. To accommodate these scenarios, two different indices for DS1 interfaces are introduced in this MIB. These indices are dsxilfIndex and dsxilineIndex.

External interface scenario: the SNMP Agent represents all managed DS1 lines as external interfaces (for example, an Agent residing on the device supporting DS1 interfaces directly):

For this scenario, all interfaces are assigned an integer value equal to ifIndex, and the following applies:

ifIndex=dsxlIfIndex=dsxlLineIndex for all interfaces. 
The dsxlIfIndex column of the DS1 Configuration table relates each DS1 interface to its corresponding interface (ifIndex) in the Internet-standard MIB (MIB-II STD 17, RFC 1213) [RFC1213].

External \& Internal interface scenario: the SNMP Agents resides on a host external from the device supporting DS1 interfaces (e.g., a router). The Agent represents both the host and the DSI device. The index dsxlLineIndex is used to not only represent the DS1 interfaces external from the host/DS1-device combination, but also the DS1 interfaces connecting the host and the DS1 device. The index dsxlIfIndex is always equal to ifIndex.

Example:

A shelf full of CSUs connected to a router. An SNMP Agent residing on the router proxies for itself and the CSU. The router has also an Ethernet interface:

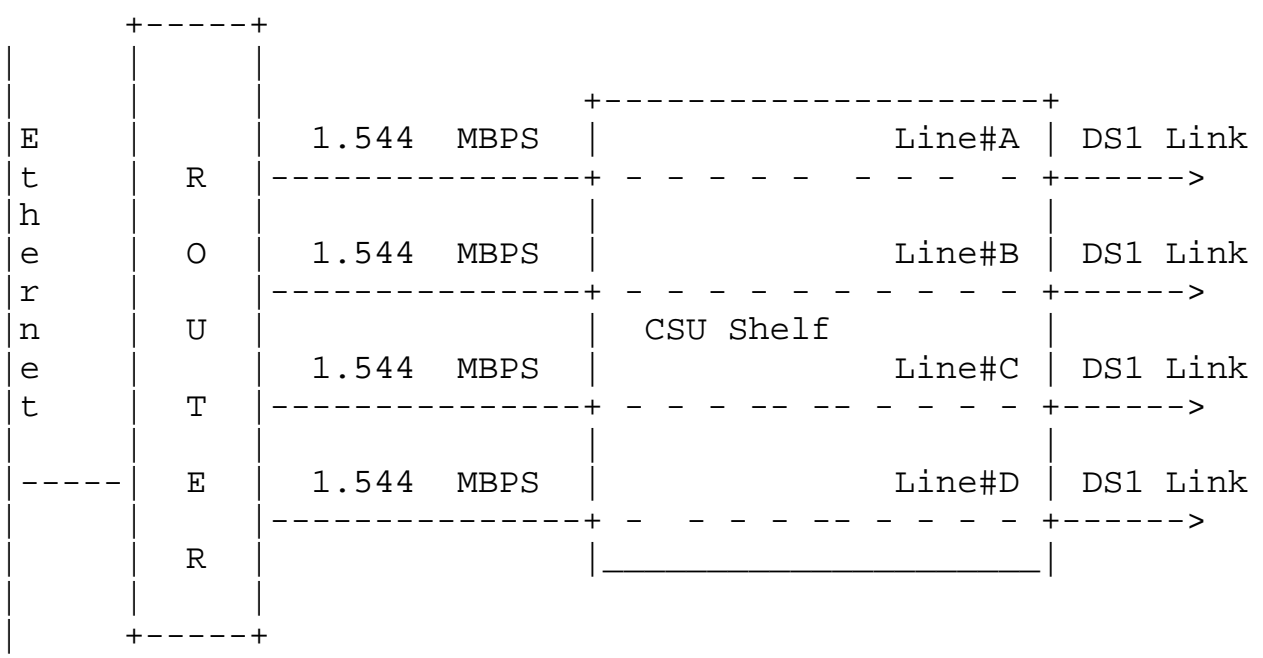

The assignment of the index values could for example be:

\begin{tabular}{cclc} 
ifIndex & $(=$ dsxIIfIndex $)$ & \multicolumn{1}{c}{ NA } & dsxiLineIndex \\
1 & Line\#A & Router Side & NA (Ethernet) \\
2 & Line\#A & Network Side & 6 \\
2 & Line\#B & Router Side & 8 \\
3 & Line\#B & Network Side & 9 \\
3 & Line\#C & Router Side & 10 \\
4 & Line\#C & Network Side & 11 \\
4 & Line\#D & Router Side & 12 \\
5 & Line\#D & Network Side & 13 \\
5 & & &
\end{tabular}


For this example, ifNumber is equal to 5. Note the following description of dsxllineIndex: the dsxlLineIndex identifies a DS1 Interface on a managed device. If there is an ifEntry that is directly associated with this and only this DS1 interface, it should have the same value as ifIndex. Otherwise, number the dsxllineIndices with an unique identifier following the rules of choosing a number greater than ifNumber and numbering inside interfaces (e.g., equipment side) with even numbers and outside interfaces (e.g., network side) with odd numbers.

If the CSU shelf is managed by itself by a local SNMP Agent, the situation would be:

$\begin{array}{ccll}\text { ifIndex } & (=\text { dsxiffindex }) & & \text { dsxiLineIndex } \\ 1 & \text { Line\#A } & \text { Network Side } & 1 \\ 2 & \text { Line\#A } & \text { RouterSide } & 2 \\ 3 & \text { Line\#B } & \text { Network Side } & 3 \\ 4 & \text { Line\#B } & \text { RouterSide } & 4 \\ 5 & \text { Line\#C } & \text { Network Side } & 5 \\ 6 & \text { Line\#C } & \text { Router Side } & 6 \\ 7 & \text { Line\#D } & \text { Network Side } & 7 \\ 8 & \text { Line\#D } & \text { Router Side } & 8\end{array}$

Appendix B - The Delay Approach to Unavailable Seconds

This procedure is illustrated below for a DS1 ESF interface. Similar rules would apply for other DS1, DS2, and E1 interface variants. The procedure guarantees that the statistical counters are correctly updated at all times, although they lag real time by 10 seconds. At the end of each 15-minute interval, the current interval counts are transferred to the most recent interval entry and each interval is shifted up by one position, with the oldest being discarded if necessary in order to make room. The current interval counts then start over from zero. Note, however, that the signal state calculation does not start afresh at each interval boundary; rather, signal state information is retained across interval boundaries. 


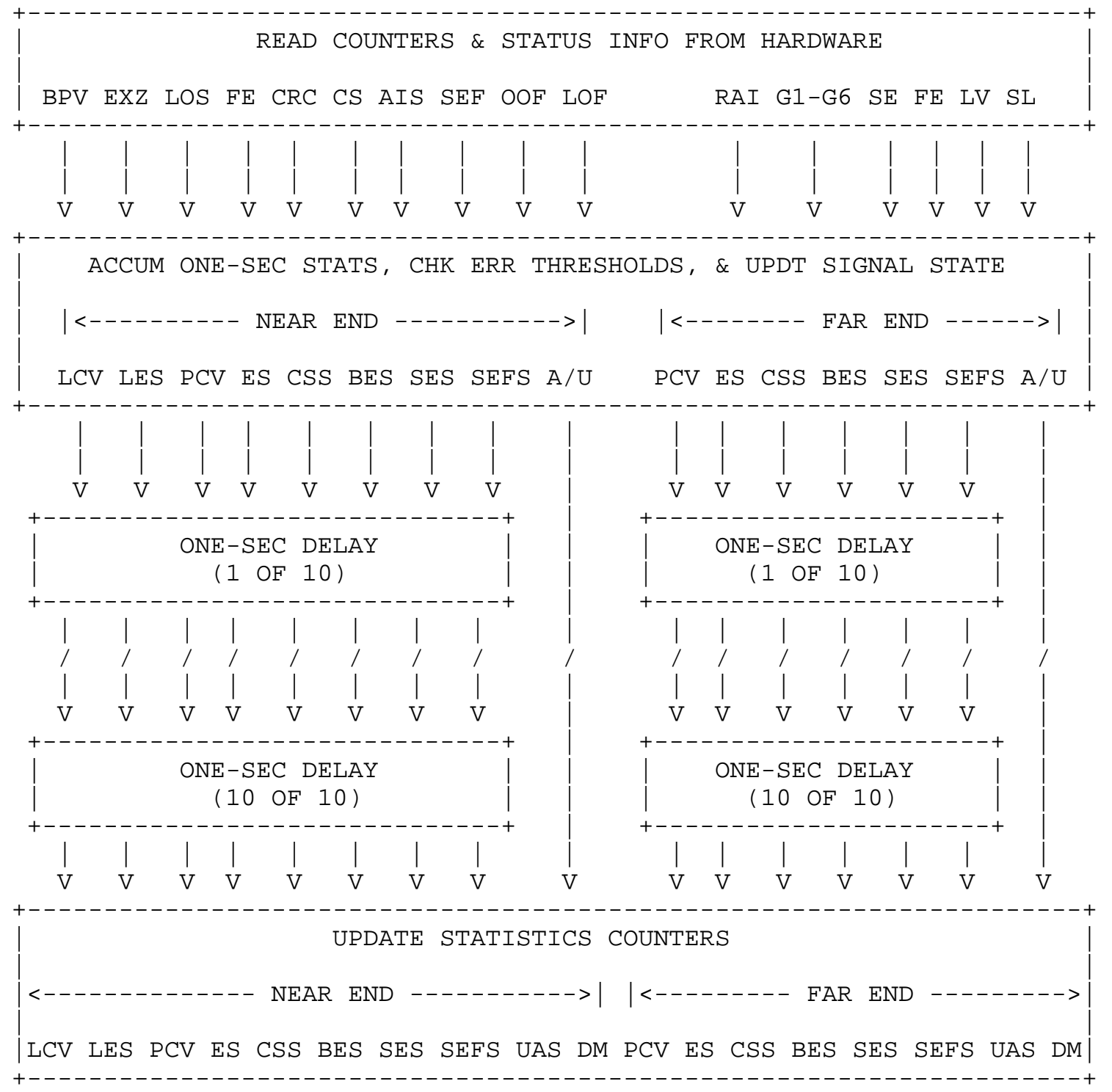

Note that if such a procedure is adopted, there is no current interval data for the first 10 seconds after a system comes up. noSuchInstance must be returned if a management station attempts to access the current interval counters during this time.

It is an implementation-specific matter whether an agent assumes that the initial state of the interface is available or unavailable. 
Appendix C - Changes from Pervious Versions

C.1. Changes from RFC 3895

The changes from RFC 3895 [RFC3895] are the following:

(1) Values were added to dsxlLineType to support J1 types.

(2) The object dsxllineImpedance was added.

(3) All DM-related objects were deprecated following their removal from ITU performance standards.

(4) Relevant text and reference section were updated.

(5) Changes in Compliance Statements to include new values.

C.2. Changes from RFC 2495

The changes from RFC 2495 [RFC2495] are the following:

(1) The dsxlFracIfIndex SYNTAX matches the description range.

(2) A value was added to dsxlTransmitclocksource.

(3) Values were added to dsxllinetype.

(4) Two objects were added, dsxlLineMode and dsxlLineBuildout, to better express transceiver mode and LineBuildout for T1.

(5) Reference was added to Circuit Identifier object.

(6) Align the DESCRIPTION clauses of few statistic objects with the near-end definition, with the far-end definition, and with [RFC3593].

(7) Changes in Compliance Statements to include new objects.

(8) A typographical error in dsx2E2 was fixed; new name is dsx1E2.

C.3. Changes from RFC 1406

The changes from RFC 1406 [RFC1406] are the following:

(1) The Fractional table has been deprecated.

(2) This document uses SMIv2.

(3) Usage is given for ifTable and ifXTable.

(4) Example usage of ifstackTable is included.

(5) dsxiIfIndex has been deprecated.

(6) Support for DS2 and E2 has been added.

(7) Additional lineTypes for DS2, E2, and unframed E1 were added.

(8) The definition of valid intervals has been clarified for the case where the agent proxied for other devices. In particular, the treatment of missing intervals has been clarified.

(9) An inward loopback has been added.

(10) Additional linestatus bits have been added for Near End in Unavailable Signal State, Carrier Equipment Out of Service, DS2 Payload AIS, and DS2 Performance Threshold. 
(11) A read-write line Length object has been added.

(12) Signal mode of other has been added.

(13) Added a linestatus last change, trap and enabler.

(14) The el(19) iftype has been obsoleted, so this MIB does not list it as a supported iftype.

(15) Textual Conventions for statistics objects have been used.

(16) A new object, dsx1Loopbackstatus, has been introduced to reflect the loopbacks established on a DS1 interface and the source to the requests. dsxlLoopbackConfig continues to be the desired loopback state while dsxlLoopbackstatus reflects the actual state.

(17) A dual loopback has been added to allow the setting of an inward loopback and a line loopback at the same time.

(18) An object indicating which channel to use within a parent object (i.e., DS3) has been added.

(19) An object has been added to indicate whether or not this DS1/E1 is channelized.

(20) Line coding type of B6ZS has been added for DS2.

\section{C.4. Companion Documents}

This document is a companion to the documents that define managed objects for the DSO [RFC2494], DS3/E3 [RFC3896], and Synchronous Optical Network/Synchronous Digital Hierarchy (SONET/SDH) [RFC3592] Interface Types.

Author's Address

Orly Nicklass, Editor

RAD Data Communications, Ltd.

Ziv Tower, 24 Roul Walenberg

Tel Aviv, Israel, 69719

Phone: 9723-765-9969

EMail: orly_n@rad.com 
Full Copyright statement

Copyright (C) The IETF Trust (2007).

This document is subject to the rights, licenses and restrictions contained in BCP 78, and except as set forth therein, the authors retain all their rights.

This document and the information contained herein are provided on an "AS IS" basis and THE CONTRIBUTOR, THE ORGANIZATION HE/SHE REPRESENTS OR IS SPONSORED BY (IF ANY), THE INTERNET SOCIETY, THE IETF TRUST AND THE INTERNET ENGINEERING TASK FORCE DISCLAIM ALL WARRANTIES, EXPRESS OR IMPLIED, INCLUDING BUT NOT LIMITED TO ANY WARRANTY THAT THE USE OF THE INFORMATION HEREIN WILL NOT INFRINGE ANY RIGHTS OR ANY IMPLIED WARRANTIES OF MERCHANTABILITY OR FITNESS FOR A PARTICULAR PURPOSE.

Intellectual Property

The IETF takes no position regarding the validity or scope of any Intellectual property Rights or other rights that might be claimed to pertain to the implementation or use of the technology described in this document or the extent to which any license under such rights might or might not be available; nor does it represent that it has made any independent effort to identify any such rights. Information on the procedures with respect to rights in RFC documents can be found in BCP 78 and BCP 79 .

Copies of IPR disclosures made to the IETF Secretariat and any assurances of licenses to be made available, or the result of an attempt made to obtain a general license or permission for the use of such proprietary rights by implementers or users of this specification can be obtained from the IETF on-line IPR repository at http://www.ietf.org/ipr.

The IETF invites any interested party to bring to its attention any copyrights, patents or patent applications, or other proprietary rights that may cover technology that may be required to implement this standard. Please address the information to the IETF at ietf-ipreietf.org.

Acknowledgement

Funding for the RFC Editor function is currently provided by the Internet Society. 